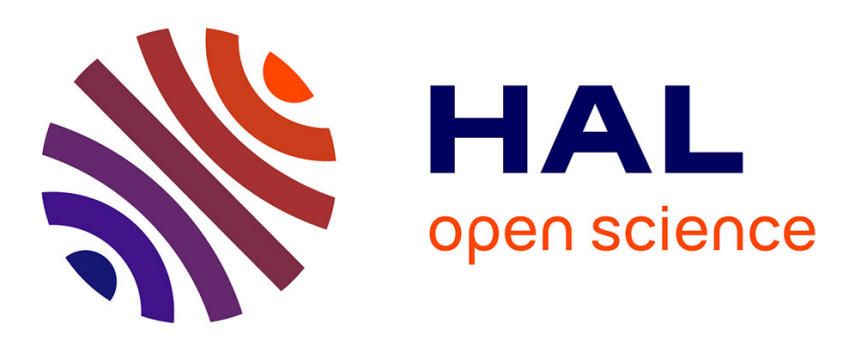

\title{
Effective wave motion in periodic discontinua near spectral singularities at finite frequencies and wavenumbers
}

\author{
Bojan B Guzina, Marc Bonnet
}

\section{To cite this version:}

Bojan B Guzina, Marc Bonnet. Effective wave motion in periodic discontinua near spectral singularities at finite frequencies and wavenumbers. Wave Motion, 2021, 103, pp.102729. 10.1016/j.wavemoti.2021.102729 . hal-03184997

\section{HAL Id: hal-03184997 https://hal.science/hal-03184997}

Submitted on 30 Mar 2021

HAL is a multi-disciplinary open access archive for the deposit and dissemination of scientific research documents, whether they are published or not. The documents may come from teaching and research institutions in France or abroad, or from public or private research centers.
L'archive ouverte pluridisciplinaire HAL, est destinée au dépôt et à la diffusion de documents scientifiques de niveau recherche, publiés ou non, émanant des établissements d'enseignement et de recherche français ou étrangers, des laboratoires publics ou privés. 


\title{
Effective wave motion in periodic discontinua near spectral singularities at finite frequencies and wavenumbers
}

\author{
Bojan B. Guzina ${ }^{\mathrm{a}, *}$, Marc Bonnet ${ }^{\mathrm{b}}$ \\ ${ }^{a}$ Department of Civil, Environmental, and Geo-Engineering, University of Minnesota, Twin Cities, United States \\ (guzin001@umn.edu) \\ ${ }^{b}$ POEMS (CNRS-INRIA-ENSTA Paris), Applied Mathematics Department, ENSTA Paris, Palaiseau, France \\ (mbonnet@ensta.fr)
}

\begin{abstract}
We consider the effective wave motion, at spectral singularities such as corners of the Brillouin zone and Dirac points, in periodic continua intercepted by compliant interfaces that pertain to e.g. masonry and fractured materials. We assume the Bloch-wave form of the scalar wave equation (describing anti-plane shear waves) as a point of departure, and we seek an asymptotic expansion about a reference point in the wavenumber-frequency space - deploying wavenumber separation as the perturbation parameter. Using the concept of broken Sobolev spaces to cater for the presence of kinematic discontinuities, we next define the "mean" wave motion via inner product between the Bloch wave and an eigenfunction (at specified wavenumber and frequency) for the unit cell of periodicity. With such projection-expansion approach, we obtain an effective field equation, for an arbitrary dispersion branch, near apexes of "wavenumber quadrants" featured by the first Brillouin zone. For completeness, we investigate asymptotic configurations featuring both (a) isolated, (b) repeated, and (c) nearby eigenvalues. In the case of repeated eigenvalues, we find that the "mean" wave motion is governed by a system of wave equations and Dirac equations, whose size is given by the eigenvalue multiplicity, and whose structure is determined by the participating eigenfunctions, the affiliated cell functions, and the direction of wavenumber perturbation. One of these structures is shown to describe the so-called Dirac points - apexes of locally conical dispersion surfaces - that are relevant to the generation of topologically protected waves. In situations featuring clusters of tightly spaced eigenvalues, the effective model is found to entail a Diraclike system of equations that generates "blunted" conical dispersion surfaces. We illustrate the analysis by numerical simulations for two periodic configurations in $\mathbb{R}^{2}$ that showcase the asymptotic developments in terms of (i) wave dispersion, (ii) forced wave motion, and (iii) frequency- and wavenumber-dependent phonon behavior.
\end{abstract}

Keywords: waves in periodic media; dynamic homogenization; finite wavenumber; finite frequency; discontinuous media; Dirac points

\section{Introduction}

The long-standing interest in developing effective continuum models of piecewise-continuous systems, such as masonry and (geo-) materials intercepted by quasi-periodic systems of fractures, has been driven by a number of factors - most notably the need for deeper insight into the mechanical behavior of such quintessential material structures, and the complexity of numerical simulations due to the need of interface elements. An early effort toward achieving this goal can be found in [26], seeking the effective anisotropic elasticity of fractured rock - under static conditions - by decomposing its compliance tensor into that of (i) the intact rock, and (ii) the network of fractures. In parallel a mechanistic approach to the homogenization of periodic brickwork, that amounts to an imposition of the Hill-Mandel macro-homogeneity condition, was proposed in [1]. For a comprehensive review of the elastostatic continuum descriptions of masonry, including enriched models, the reader is referred to $[25,31,13]$.

${ }^{*}$ Corresponding author 
In terms of dynamic masonry behavior, a Cosserat continuum description of (one- and two-dimensional) periodic block structures was obtained in $[22,30]$ by converting the governing discrete equations into respective differential equations via continuation and Taylor expansion. Thanks to the intrinsic length scale(s) featured by the Cosserat continuum, this class of effective models were shown to capture the dispersive wave motion in masonry-like structures at wavelengths exceeding 4-5 block sizes. Such general approach to the longwavelength homogenization of block structures was extended in [28], by way of energy considerations, toward three-dimensional dynamic analysis of masonry structures. Motivated by the fact that the Cosserat continuum is insufficient to describe the six degrees of freedom featured by diatomic masonry patterns, the latter study was followed by a higher-order micromorphic continuum model of interlocking block structures [29]. In terms of fractured rock masses, on the other hand, effective models for the low-frequency dispersion of waves in poroelastic continua intercepted by a periodic system of parallel fractures were considered for instance in $[6,14]$. A two-scale approach to the homogenization of periodic media - that combines asymptotic and variational methods [27] - has been applied, up to the second order, toward numerical continuum description of masonry structures in [3]. Like the aforementioned techniques, this approach assumes long-wavelength, low-frequency wave motion in block structures.

Over the past two decades, however, a mathematical framework for the finite-wavenumber, finite-frequency (FW-FF) homogenization of periodic media - near spectral singularities such as corners of the Brillouin zone $[11,5,32,9,16]$ - has emerged as a viable tool for an effective asymptotic description of the germane wave phenomena that include not only dispersion, but also negative refraction, band gaps, and Dirac cones among others. In the core of this paradigm is an understanding that the energy in periodic media is carried by phase-delayed internal resonances (the so-called phonons) - which then implies a new definition of the mean motion as a projection of the "rough" wavefield onto suitable eigenfunction(s) for the unit cell of periodicity. Inherently, these eigenfunctions are computed at a particular wavenumber (inside the first Brillouin zone) and eigenfrequency, signifying the origin of the asymptotic expansion.

In this vein, time appears ripe for developing the FW-FF continuum description of waves in periodic block structures. As a point of departure, we consider the scalar wave equation in $\mathbb{R}^{d}(d \geqslant 1)$, and we adopt the recent approach [16] that merges the concepts of two-scale asymptotic expansion [4] and Blochwave description as natural grounds for homogenization analysis in the wavenumber-frequency space [20]. To facilitate the application to piecewise-continuous systems, we make use of the broken periodic Sobolev spaces, and we assume a linear contact model for the lines (or surfaces) of discontinuity - relating the displacement jump to the surface traction. In this setting we demonstrate, assuming the reference eigenfrequency to be simple, that the effective "masonry" model is (to the leading order) governed by an anisotropic wave equation, whose effective elasticity tensor and mass density are computed in terms of the germane eigenfunction and the so-called first cell function, also defined over the unit cell of periodicity. By extending such asymptotic analysis to the next order, we obtain an enriched (fourth-order) governing equation that bears semblance to the models of gradient elasticity, but whose coefficients are directly (and uniquely) computable from the structure of the unit cell. For generality, we also consider the situations of repeated eigenvalues, in which case the wave motion is governed by a system of either wave equations or Dirac equations, depending on the spectral properties of a particular matrix whose entries depend on the participating eigenfunctions, the properties of the unit cell, and the direction of perturbation in the wavenumber space. For generality, following [20, 16] we also provide the homogenization ansatz for the source term (when non-trivial), and we consider a degenerate case of closely spaced eigenvalues that is directly relevant to the phenomenon of topologically protected waves [21]. We illustrate the analytical developments by numerical simulations for two example configurations in $\mathbb{R}^{2}$ that (i) showcase the metamaterial design using soft interfaces, and (ii) demonstrate the occurence of band gaps and Dirac-like dispersion structures in masonry-like discontinua.

\section{Preliminaries}

With reference to a Cartesian frame tied to an orthonormal vector basis $\boldsymbol{e}_{j}(j=\overline{1, d})$, consider the timeharmonic wave equation

$$
\begin{gathered}
-\nabla \cdot(G \nabla u)-\omega^{2} \rho u=f \quad \text { in } \mathbb{R}^{d} \backslash \Gamma_{\mathbb{R}}, \\
\llbracket t_{\boldsymbol{\nu}}[u] \rrbracket=0, \quad \llbracket u \rrbracket+\kappa^{-1} t_{\boldsymbol{\nu}}[u]=0 \quad \text { on } \Gamma_{\mathbb{R}}
\end{gathered}
$$


at frequency $\omega$, where $G>0$ and $\rho>0$ are constants; $f=f(\boldsymbol{x})$ is the source term; $\Gamma_{\mathbb{R}}$ is a $(d-1)$-dimensional, $Y$-periodic system of interfaces spanning $\mathbb{R}^{d}$;

$$
Y=\left\{\boldsymbol{x}: 0<\boldsymbol{x} \cdot \boldsymbol{e}_{j}<\ell_{j} ; j=\overline{1, d}\right\}
$$

is the periodicity cell; $\kappa \in L^{\infty}(\Gamma)$, with $\Gamma=\Gamma_{\mathbb{R}} \cap Y$, is a positive $Y$-periodic function;

$$
t_{\boldsymbol{\nu}}[g](\boldsymbol{x})=\boldsymbol{\nu}(\boldsymbol{x}) \cdot G \nabla g(\boldsymbol{x}) ;
$$

$\boldsymbol{\nu}$ is the unit normal on $\Gamma_{\mathbb{R}}$ with arbitrary but fixed orientation, and

$$
\llbracket g \rrbracket(\boldsymbol{x}):=\lim _{\delta \rightarrow 0}(g(\boldsymbol{x}+\delta \boldsymbol{\nu}(\boldsymbol{x}))-g(\boldsymbol{x}-\delta \boldsymbol{\nu}(\boldsymbol{x}))), \quad \boldsymbol{x} \in \Gamma_{\mathbb{R}} .
$$

In what follows, we conveniently assume that

$$
|\Gamma \cap \partial Y|=0 \text { in } \mathbb{R}^{d-1} .
$$

Remark 1. When $d=2$, (1) permits interpretation in the context of elasticity and anti-plane shear waves. In this case $u, G, \rho, \kappa$ and $f$ take respectively the roles of transverse displacement, shear modulus, mass density, specific contact stiffness, and body force. In situations when the reference elastic solid is further anisotropic, (1)-(3) and the ensuing formulation can be generalized by replacing $G$ with a positive definite second-order tensor $\boldsymbol{G}$. By letting $\kappa \rightarrow 0 \Gamma_{\mathbb{R}}$ becomes a traction-free crack, while $\kappa \rightarrow \infty$ achieves perfect bonding on $\Gamma_{\mathbb{R}}$. Alternatively, (1) also applies (in any space dimension) to linear acoustic media characterized by the bulk modulus $B$ and the mass density $\varrho$, with the replacements $\rho \rightarrow B^{-1}$ and $G \rightarrow \varrho^{-1}$. In that case, u takes the role of pressure, $(\mathrm{i} \omega)^{-1} \boldsymbol{\nu} \cdot \nabla u$ is the normal velocity, and the limiting situation $\kappa=0$ corresponds to $\Gamma_{\mathbb{R}}$ behaving as a rigid screen.

\subsection{Bloch wave representation}

Recalling the plane wave expansion approach [23, 24], we seek the Bloch-wave solutions of (1) i.e.

$$
u(\boldsymbol{x})=\tilde{u}(\boldsymbol{x}) e^{i \boldsymbol{k} \cdot \boldsymbol{x}}, \quad \tilde{u}: Y \text {-periodic }
$$

where $\tilde{u}$ depends implicitly on $\boldsymbol{k} \in \mathbb{R}^{d}$ and $\omega \in \mathbb{R}$ - which are taken as fixed. Letting the source term likewise take the Bloch-wave form $f(\boldsymbol{x})=\tilde{f}(\boldsymbol{x}) e^{i \boldsymbol{k} \cdot \boldsymbol{x}}$ with $\tilde{f} \in L^{2}(Y)$, (1) becomes

$$
\begin{array}{rlrlrl}
-\nabla_{\boldsymbol{k}} \cdot\left(G \nabla_{\boldsymbol{k}} \tilde{u}\right)-\omega^{2} \rho \tilde{u} & =\tilde{f} & & \text { in } Y, \\
\llbracket t_{\boldsymbol{\nu}}^{\boldsymbol{k}}[\tilde{u}, \tilde{u}] \rrbracket=0, & \llbracket \tilde{u} \rrbracket+\kappa^{-1} t_{\boldsymbol{\nu}}^{\boldsymbol{k}}[\tilde{u}, \tilde{u}] & =0 & & \text { on } \Gamma, \\
1 \tilde{u}\rfloor_{j}^{-}=0, & \left.1 t_{\boldsymbol{n}}^{\boldsymbol{k}}[\tilde{u}, \tilde{u}]\right|_{j} ^{+}=0 \quad j & =\overline{1, d} & & \text { on } \partial Y,
\end{array}
$$

where $\nabla_{\boldsymbol{k}}=\nabla+i \boldsymbol{k} ; x_{j}=\boldsymbol{x} \cdot \boldsymbol{e}_{j} ; \boldsymbol{n}$ is the unit outward normal on $\partial Y$;

$$
t_{\boldsymbol{\nu}}^{\boldsymbol{k}}[g, h](\boldsymbol{x})=\boldsymbol{\nu}(\boldsymbol{x}) \cdot G(\nabla g(\boldsymbol{x})+i \boldsymbol{k} h(\boldsymbol{x})),
$$

and the assumed $Y$-periodicity of $\tilde{u}$ is conveniently expressed using the notation

$$
\left.1 g\right|_{j} ^{ \pm}(\boldsymbol{x}):=\left.g(\boldsymbol{x})\right|_{x_{j}=0} \pm\left. g(\boldsymbol{x})\right|_{x_{j}=\ell_{j}}, \quad j=\overline{1, d} .
$$

With reference to Fig. 1 which illustrates the unit cell $Y$, we conveniently introduce $\Gamma^{+}$as an extension of $\Gamma$ such that every smooth segment of $\Gamma^{+}$terminates on $\partial Y$; inherently, one has

$$
\llbracket \tilde{u} \rrbracket=0, \quad \llbracket t_{\nu}^{\boldsymbol{k}}[\tilde{u}, \tilde{u}] \rrbracket=0 \quad \text { on } \Gamma^{+} \backslash \Gamma .
$$

On denoting by $Y_{\mathfrak{m}}(\mathfrak{m}=\overline{1, \mathfrak{M}})$ the contiguous open subdomains of $Y$ separated by $\Gamma^{+}$, we have

$$
\bar{Y}=\cup \overline{Y_{\mathfrak{m}}},
$$

and we hereon assume that each $Y_{\mathfrak{m}}$ is a Lipschitz domain.

Remark 2. Throughout the remainder of this work, the conormal traces $t_{\boldsymbol{\nu}}$ and $t_{\boldsymbol{\nu}}^{\boldsymbol{k}}$ are continuous across $\Gamma$. As a result, the statements $\llbracket t_{\boldsymbol{\nu}}[\cdot] \rrbracket=\mathbf{0}$ and $\llbracket t_{\boldsymbol{\nu}}^{\boldsymbol{k}}[\cdot, \cdot] \rrbracket=\mathbf{0}$ are hereon assumed implicitly. For brevity, we shall also assume each periodicity statement $\left.1 \cdot\right|_{j} ^{ \pm}=0$ on $\partial Y$ to hold for $j=\overline{1, d}$ without explicitly stating so.

Remark 3. With no additional complication, we can assume the mass density to be piecewise-constant according to

$$
\rho=\rho_{\mathfrak{m}} \quad \text { for } \boldsymbol{x} \in Y_{\mathfrak{m}},
$$

which will be our premise going forward. 


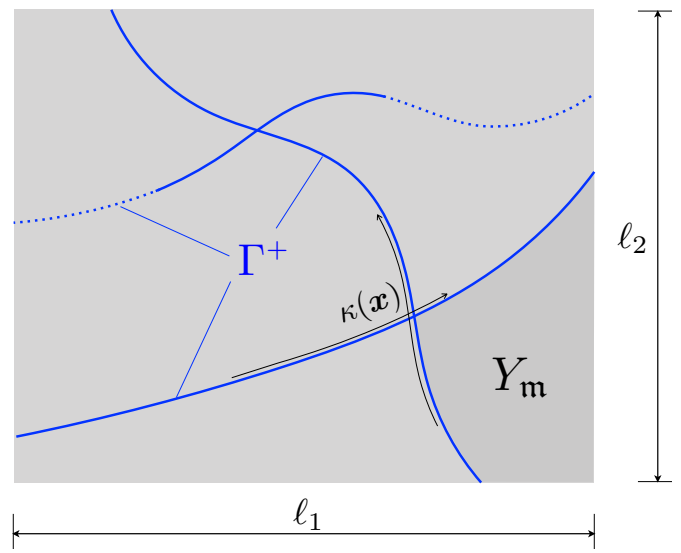

Figure 1: Schematics of the periodicity cell $Y=\cup Y_{\mathfrak{m}}$ in $\mathbb{R}^{2}$, where $Y_{\mathfrak{m}}(\mathfrak{m}=\overline{1, \mathfrak{M}})$ are contiguous open regions separated by an extended system of compliant interfaces $\Gamma^{+}$.

\section{FW-FF homogenization near apexes of the first Brillouin zone: isolated eigenvalues}

In this section, we seek an asymptotic solution of (6) assuming that the wavenumber-frequency pair $(\boldsymbol{k}, \omega)$ is in a neighborhood of a spectral singularity given by the corner (of any "quadrant") of the first Brillouin zone, at "elevation" $\lambda_{n}^{1 / 2}$ corresponding to an isolated $n$th dispersion branch. Following [16], we conclude the section by elucidating how such (Bloch-wave) solution could be used to obtain an effective description of the field equation (1) featuring a point source in the physical space.

Hereon, we make use of the $L_{\rho}^{2}$ function space

$$
L_{\rho}^{2}(Y)=\left\{g \in L^{2}(Y):(\rho g, g)<\infty\right\},
$$

and the broken [e.g. 8] periodic Sobolev space

$$
H_{p}^{1}(Y)=\left\{g \in L^{2}(Y): g \in L^{2}\left(Y_{\mathfrak{m}}\right), \nabla g \in\left(L^{2}\left(Y_{\mathfrak{m}}\right)\right)^{d}, 1 g l_{j}^{-}=0 \text { on } \partial Y, j=\overline{1, d}, \mathfrak{m}=\overline{1, \mathfrak{M}}\right\},
$$

where $(g, h):=(g, h)_{Y}=\sum_{\mathfrak{m}=1}^{\mathfrak{M}}(g, h)_{Y_{\mathfrak{m}}}$ denotes the inner product over $Y$ for $g, h \in L^{2}(Y)$. To facilitate the treatment of vector- and tensor-valued functions, we shall also write

$$
(\boldsymbol{g}, \boldsymbol{h})=\sum_{\mathfrak{m}=1}^{\mathfrak{M}} \int_{Y_{\mathfrak{m}}} \boldsymbol{g}(\boldsymbol{x}): \overline{\boldsymbol{h}}(\boldsymbol{x}) \mathrm{d} \boldsymbol{x}, \quad \boldsymbol{g} \in\left(L_{p}^{2}(Y)\right)^{d^{q}}, \quad \boldsymbol{h} \in\left(L_{p}^{2}(Y)\right)^{d^{r}}, \quad q, r \geqslant 0
$$

where ":" denotes the usual product, the inner product, and the $\min (q, r)$-tuple tensor contraction when $q=r=0, q=r=1$, and $\max (q, r)>1$, respectively. We will similarly use the notation $(g, h)_{S}$ and its tensorial version for inner products over surfaces $S \subset \mathbb{R}^{d-1}$, with e.g. $S=\partial Y, S=\cup_{\mathfrak{m}=1}^{\mathfrak{M}} \partial Y_{\mathfrak{m}}$, or $S=\Gamma$.

\subsection{Forced motion problem and eigensystem representation}

Letting $\tilde{\phi}=\tilde{\phi}(\boldsymbol{k}) \in H_{p}^{1}(Y)$ and $\tilde{\lambda}=\tilde{\lambda}(\boldsymbol{k}) \in \mathbb{C}$, we consider the eigenvalue problem

$$
\begin{aligned}
-\nabla_{\boldsymbol{k}} \cdot\left(G \nabla_{\boldsymbol{k}} \tilde{\phi}\right) & =\tilde{\lambda} \rho \tilde{\phi} \quad \text { in } Y, \\
\llbracket \tilde{\phi} \rrbracket+\kappa^{-1} t_{\boldsymbol{\nu}}^{\boldsymbol{k}}[\tilde{\phi}, \tilde{\phi}] & =0 \quad \text { on } \Gamma, \quad\left|t_{\boldsymbol{n}}^{\boldsymbol{k}}[\tilde{\phi}, \tilde{\phi}]\right|_{j}^{+}=0 \quad \text { on } \partial Y,
\end{aligned}
$$

On introducing the operator

$$
A(\boldsymbol{k}) \tilde{\phi}=-\nabla_{\boldsymbol{k}} \cdot\left(G \nabla_{\boldsymbol{k}} \tilde{\phi}(\cdot)\right),
$$

we find by integrating by parts over $Y$ that

$$
(A(\boldsymbol{k}) \tilde{\phi}, \tilde{\phi})=\left(G \nabla_{\boldsymbol{k}} \tilde{\phi}, \nabla_{\boldsymbol{k}} \tilde{\phi}\right)+(\kappa \llbracket \tilde{\phi} \rrbracket, \llbracket \tilde{\phi} \rrbracket)_{\Gamma} \geqslant C\|\tilde{\phi}\|_{H_{p}^{1}(Y)}^{2}-c(\tilde{\phi}, \tilde{\phi}),
$$


where $0<C<G$ and $c>0$ are constants independent of $\boldsymbol{k}$. From (10), it follows (assuming some fixed $c>C$ ) that the self-adjoint operator $A(\boldsymbol{k})$ has a resolvent $R_{c}(\boldsymbol{k})=(A(\boldsymbol{k})+c I)^{-1}$ that can be seen as an $L_{p}^{2}(Y) \rightarrow L_{p}^{2}(Y)$ map. Since the solution of (9) resides in $H_{p}^{1}(Y)$, the compactness of $R_{c}(\boldsymbol{k})$ follows by the compact embedding $H_{p}^{1}(Y) \hookrightarrow L_{p}^{2}(Y)$ due to Rellich theorem. By the equivalence of problems $A(\boldsymbol{k}) \tilde{\phi}=\tilde{\lambda} \rho \tilde{\phi}$ and $(c+\tilde{\lambda} \rho) R_{c}(\boldsymbol{k}) \tilde{\phi}=\tilde{\phi}$ (subject to germane boundary conditions) for $\tilde{\phi} \in H_{p}^{1}(Y)$ and $\tilde{\lambda} \in \mathbb{R}$, we find that for each $\boldsymbol{k}$ there exists eigensystem $\left\{\tilde{\phi}_{m}(\boldsymbol{k}) \in H_{p}^{1}(Y), \tilde{\lambda}_{m}(\boldsymbol{k}) \in \mathbb{R}\right\}$ that solves $(9)$, where $\left\{\tilde{\phi}_{m}\right\}$ are complete and orthogonal in $L_{\rho}^{2}(Y)$ as

$$
\left(\rho \tilde{\phi}_{m}, \tilde{\phi}_{n}\right)=0 \quad \text { for } m \neq n, \quad\left(\tilde{\phi}_{m}, \tilde{\phi}_{m}\right)=1 .
$$

We also note that $\left\{\tilde{\lambda}_{m}\right\}$ are in this case (i) continuous for all $\boldsymbol{k}$, and (ii) analytic functions of $\boldsymbol{k}$ everywhere inside the first Brillouin zone except on subsets of measure zero where their multiplicity changes [33].

As a result, any $\tilde{u} \in L_{\rho}^{2}(Y)$ can be expanded as

$$
\tilde{u}(\boldsymbol{x})=\sum_{m=1}^{\infty} \tilde{\alpha}_{m} \tilde{\phi}_{m}(\boldsymbol{x})
$$

where $\tilde{\alpha}_{m}$ are, for given $\boldsymbol{k}$, constants. By virtue of (11) and the weak statement of (6), namely

$$
-\omega^{2}\left(\rho \tilde{u}, \tilde{\phi}_{m}\right)+\left(\tilde{u}, A(\boldsymbol{k}) \tilde{\phi}_{m}\right)=\left(\tilde{f}, \tilde{\phi}_{m}\right),
$$

obtained by repeated integration by parts, we find that

$$
\tilde{u}=\sum_{m=1}^{\infty} \frac{\left(\tilde{f}, \tilde{\phi}_{m}\right)}{\left(\rho \tilde{\phi}_{m}, \tilde{\phi}_{m}\right)} \frac{1}{\tilde{\lambda}_{m}-\omega^{2}} \tilde{\phi}_{m}, \quad \text { when } \quad \omega^{2} \neq \tilde{\lambda}_{m}, \quad m \in \mathbb{N}^{+}
$$

holds in the $L_{p}^{2}(Y)$ sense, where $\mathbb{N}^{+}$is the set of positive integers.

Remark 4. From the above arguments, one finds that the necessary and sufficient condition for the unique solvability of (6) is $\omega^{2} \neq \tilde{\lambda}_{m}, \forall m$. If $\omega^{2}=\tilde{\lambda}_{n}$ for some $n$, problem (6) is still solvable provided $\left(\tilde{f}, \tilde{\phi}_{n}\right)=0$, see [20] for discussion.

\subsection{Effective wave motion}

Let $\mathcal{B}=\left\{\boldsymbol{k}:\left|k_{j}\right| \leqslant \pi / \ell_{j}, j=\overline{1, d}\right\}$ be the first Brillouin zone, i.e. the counterpart of $Y$ in the Fourier $\boldsymbol{k}$-space containing $\{\mathbf{0}\}$. In this setting, we seek an effective description of scalar waves (1)-(2) in a "discontinuum" $\mathbb{R}^{d}$ in terms of the Bloch wave contribution solving (6) within the first "quadrant" of $\mathcal{B}$, namely

$$
\mathcal{B}^{+}=\left\{\boldsymbol{k}: 0 \leqslant k_{j} \leqslant \pi / \ell_{j}, j=\overline{1, d}\right\},
$$

whose apexes are denoted by

$$
\boldsymbol{k}^{\boldsymbol{a}}=\sum_{j=1}^{d} a_{j} \frac{\pi}{\ell_{j}} \boldsymbol{e}_{j}, \quad \boldsymbol{a}=\left(a_{1}, a_{2}, \ldots a_{d}\right), \quad a_{j} \in\{0,1\} .
$$

For brevity, we will make use of the short-hand notation

$$
\tilde{\lambda}_{n}^{\boldsymbol{a}}=\tilde{\lambda}_{n}\left(\boldsymbol{k}^{\boldsymbol{a}}\right), \quad \omega_{n}^{\boldsymbol{a}}=\left(\tilde{\lambda}_{n}^{\boldsymbol{a}}\right)^{1 / 2} .
$$

The analysis of $\mathcal{B} \backslash \mathcal{B}^{+}$, as needed, can be performed in an analogous way.

Following the recent study [16] dealing with periodic continua, we pursue this goal via the asymptotic expansion of (6) by:

- assuming that the wave motion is localized spectrally in some neighborhood of the "apex" $\left(\boldsymbol{k}^{\boldsymbol{a}}, \omega_{n}^{\boldsymbol{a}}\right)$ of $\mathcal{B}^{+}$, and 
- specifying the effective motion, loosely speaking, in terms of the inner product $\left(\tilde{u}, \tilde{\phi}_{n}^{\boldsymbol{a}}\right)$, where $\tilde{\phi}_{n}^{\boldsymbol{a}}$ is the eigenfunction solving (9) with $\boldsymbol{k}=\boldsymbol{k}^{\boldsymbol{a}}$ and $\omega=\omega_{n}^{a}$. For the remainder of this section, we assume that the eigenvalue $\tilde{\lambda}_{n}^{a}$ is simple.

Remark 5. Recalling (12), we note that the above idea is motivated by the fact that the propagation of waves along (or near) any dispersion branch of a periodic medium is carried by its internal resonances [7]. Except when $\boldsymbol{k}^{\boldsymbol{a}}=\mathbf{0}$ which signifies the origin of $\mathcal{B}$, however, these internal resonances - embodied by eigenfunctions $\phi_{n}^{\boldsymbol{a}}$ - represent waveforms that are neither standing nor $Y$-periodic. To aid the homogenization analysis, we next introduce the factorization of germane eigenfunctions [16] and we recall the idea of multi-cell analysis [32], that jointly facilitate the computation of effective wave motion as a projection of $\tilde{u}$ onto a certain periodic, standing-wave function synthesizing the internal resonances near $\left(\boldsymbol{k}^{\boldsymbol{a}}, \omega_{n}^{\boldsymbol{a}}\right)$.

\subsection{Projection basis}

At each vertex of $\mathcal{B}^{+}$, eigenfunction $\tilde{\phi}_{n}^{\boldsymbol{a}}=\tilde{\phi}_{n}\left(\boldsymbol{k}^{\boldsymbol{a}}\right) \in H_{p}^{1}(Y)$ satisfies

$$
\begin{aligned}
-\left(\nabla+i \boldsymbol{k}^{\boldsymbol{a}}\right) \cdot\left(G\left(\nabla+i \boldsymbol{k}^{\boldsymbol{a}}\right) \tilde{\phi}_{n}^{\boldsymbol{a}}\right)=\tilde{\lambda}_{n}^{\boldsymbol{a}} \rho \tilde{\phi}_{n}^{\boldsymbol{a}} \quad \text { in } Y, & \\
\llbracket \tilde{\phi}_{n}^{a} \rrbracket+\kappa^{-1} t_{\nu}^{\boldsymbol{k}^{\boldsymbol{a}}}\left[\tilde{\phi}_{n}^{\boldsymbol{a}}, \tilde{\phi}_{n}^{\boldsymbol{a}}\right]=0 \quad \text { on } \Gamma, \quad 1 t_{n}^{\boldsymbol{k}^{a}}\left[\tilde{\phi}_{n}^{\boldsymbol{a}}, \tilde{\phi}_{n}^{\boldsymbol{a}}\right] \mathrm{l}_{j}^{+}=0 & \text { on } \partial Y .
\end{aligned}
$$

Letting

$$
\tilde{\phi}_{n}^{a}(\boldsymbol{x})=\tilde{\varphi}_{n}^{\boldsymbol{a}}(\boldsymbol{x}) e^{-i \boldsymbol{k}^{\boldsymbol{a}} \cdot \boldsymbol{x}}, \quad \tilde{\phi}_{n}^{\boldsymbol{a}} \in H_{p}^{1}(Y)
$$

as in [16], and noting with reference to $(3),(4)$ and (7) that

$$
\llbracket \tilde{\phi}_{n}^{a} \rrbracket=\llbracket \tilde{\varphi}_{n}^{a} \rrbracket e^{-i \boldsymbol{k}^{a} \cdot \boldsymbol{x}}, \quad t_{\boldsymbol{\nu}}^{\boldsymbol{k}^{a}}\left[\tilde{\phi}_{n}^{\boldsymbol{a}}, \tilde{\phi}_{n}^{\boldsymbol{a}}\right]=t_{\boldsymbol{\nu}}\left[\tilde{\varphi}_{n}^{\boldsymbol{a}}\right] e^{-i \boldsymbol{k}^{a} \cdot \boldsymbol{x}} \quad \text { on } \Gamma,
$$

we obtain

$$
\begin{gathered}
-\nabla \cdot\left(G \nabla \tilde{\varphi}_{n}^{a}\right)=\tilde{\lambda}_{n}^{a} \rho \tilde{\varphi}_{n}^{a} \quad \text { in } Y, \\
\llbracket \tilde{\varphi}_{n}^{a} \rrbracket+\kappa^{-1} t_{\nu}\left[\tilde{\varphi}_{n}^{a}\right]=0 \quad \text { on } \Gamma,\left.\quad 1 \tilde{\varphi}_{n}^{a}\right|_{j} ^{(-1)^{a_{j}+1}}=0,\left.\quad 1 t_{n}\left[\tilde{\varphi}_{n}^{a}\right]\right|_{j} ^{(-1)^{a_{j}}}=0 \quad \text { on } \partial Y .
\end{gathered}
$$

Here we denote symbolically $(-1)^{m} \rightarrow$ " $"$ " (resp. $(-1)^{m} \rightarrow$ "+") for $m=1$ (resp. $\left.m=0\right)$, stating that $\tilde{\varphi}_{n}^{a}$ is $Y$-"anti-periodic" (resp. $Y$-periodic) in the $j$ th coordinate direction when $a_{j}=1$ (resp. $a_{j}=0$ ). Motivated by the multi-field continuum theory for two-dimensional crystal lattices [32], we next introduce the multi-cell domains

$$
Y_{\boldsymbol{a}}=\left\{\boldsymbol{x}: 0<\boldsymbol{x} \cdot \boldsymbol{e}_{j}<\left(1+a_{j}\right) \ell_{j} ; j=\overline{1, d}\right\}, \quad\left|Y_{\boldsymbol{a}}\right|=\prod_{j=1}^{d}\left(1+a_{j}\right),
$$

and we extend the domain of $\tilde{\varphi}_{n}^{a}$ to $Y_{\boldsymbol{a}}$ as

$$
\forall \boldsymbol{x}^{\prime} \in Y, \quad \forall T \subseteq \overline{1, d} \quad \Longrightarrow \quad \tilde{\varphi}_{n}^{\boldsymbol{a}}(\boldsymbol{x})=(-1)^{\sum_{j \in T} a_{j}} \tilde{\varphi}_{n}^{\boldsymbol{a}}\left(\boldsymbol{x}^{\prime}\right), \quad \boldsymbol{x}=\boldsymbol{x}^{\prime}+\sum_{j \in T} a_{j} \ell_{j} \boldsymbol{e}_{j} \in Y_{\boldsymbol{a}}
$$

On the basis of (16) and (17), $\tilde{\varphi}_{n}^{a} \in H_{p}^{1}\left(Y_{\boldsymbol{a}}\right)$ is found to satisfy

$$
\begin{gathered}
-\nabla \cdot\left(G \nabla \tilde{\varphi}_{n}^{a}\right)=\tilde{\lambda}_{n}^{a} \rho \tilde{\varphi}_{n}^{\boldsymbol{a}} \quad \text { in } Y_{\boldsymbol{a}}, \\
\llbracket \tilde{\varphi}_{n}^{a} \rrbracket+\kappa^{-1} t_{\boldsymbol{\nu}}\left[\tilde{\varphi}_{n}^{\boldsymbol{a}}\right]=0 \quad \text { on } \Gamma_{\boldsymbol{a}},\left.\quad 1 t_{\boldsymbol{n}}\left[\tilde{\varphi}_{n}^{\boldsymbol{a}}\right]\right|_{\boldsymbol{a}, j} ^{+}=0 \quad \text { on } \partial Y_{\boldsymbol{a}},
\end{gathered}
$$

where $\Gamma_{\boldsymbol{a}}:=\Gamma_{\mathbb{R}} \cap Y_{\boldsymbol{a}}$ collects all interfaces lying in the multi-cell domain $Y_{\boldsymbol{a}}$ and

$$
\left.1 g\right|_{\boldsymbol{a}, j} ^{ \pm}(\boldsymbol{x})=\left.g(\boldsymbol{x})\right|_{x_{j}=0} \pm\left. g(\boldsymbol{x})\right|_{x_{j}=\left(1+a_{j}\right) \ell_{j}}, \quad j=\overline{1, d} .
$$

Remark 6. We note that in directions $x_{j}$ where $a_{j}=1$, (18) are subject to the internal constraint $\left.\tilde{\varphi}_{n}^{a}\right|_{x_{j}=0}=$ $-\left.\tilde{\varphi}_{n}^{a}\right|_{x_{j}=\ell_{j}}$ which excludes those eigenfunctions of $Y_{\boldsymbol{a}}$ that are not " $Y$-antiperiodic" in the sense of (17). In what follows, this type of constraint is implicitly assumed for all boundary value problems over $Y_{\boldsymbol{a}}$. 
By recalling (15) and the normalization of $\tilde{\phi}_{n}$ that $\left(\tilde{\phi}_{n}^{\boldsymbol{a}}, \tilde{\phi}_{n}^{\boldsymbol{a}}\right)=\left(\tilde{\varphi}_{n}^{\boldsymbol{a}}, \tilde{\varphi}_{n}^{\boldsymbol{a}}\right)=1$. Thanks to this result and (11), we find that

$$
\left(\rho \tilde{\varphi}_{m}^{a}, \tilde{\varphi}_{n}^{a}\right)_{Y_{a}}=0 \quad \text { for } m \neq n, \quad\left(\tilde{\varphi}_{n}^{a}, \tilde{\varphi}_{n}^{a}\right)_{Y_{a}}=\left|Y_{a}\right|,
$$

where $(\cdot, \cdot)_{Y_{\boldsymbol{a}}}$ denotes the inner product over $Y_{\boldsymbol{a}}$.

Lemma 1. Eigenfunctions $\tilde{\varphi}_{n}^{a} \in H_{p}^{1}\left(Y_{\boldsymbol{a}}\right)$ solving (18) have constant phase, and can be taken real-valued. See Appendix, Section 7 for proof.

Remark 7. By (18) and Lemma 1, we see that the eigenfunction $\tilde{\phi}_{n}^{a}$ signifies a plane wave propagating the "standing" template $\tilde{\varphi}_{n}^{a}$ in direction $-\boldsymbol{k}^{a}$. When $\boldsymbol{k}^{\boldsymbol{a}}=\mathbf{0}, \tilde{\phi}_{n}^{\boldsymbol{a}}=\tilde{\varphi}_{n}^{a}$ is a standing wave.

Definition 1. Following [16], we introduce the effective wave motion near vertex a of $\mathcal{B}^{+}$as

$$
\langle\tilde{u}\rangle_{\boldsymbol{a}}=\frac{1}{\left|Y_{\boldsymbol{a}}\right|}\left(\tilde{u}, \tilde{\phi}_{n}^{\boldsymbol{a}}\right)_{Y_{\boldsymbol{a}}}:=\left(\tilde{u}, \tilde{\phi}_{n}^{\boldsymbol{a}}\right)_{\overline{Y_{a}}}, \quad n \geqslant 1,
$$

where $\tilde{\phi}_{n}^{a}$ is extended to $Y_{\boldsymbol{a}} \supset Y$ by the application of $Y$-periodicity. This definition is complemented by an auxiliary smoothing operator

$$
\langle\tilde{u}\rangle_{\boldsymbol{a}}^{\varphi}=\frac{1}{\left|Y_{\boldsymbol{a}}\right|}\left(\tilde{u}, \tilde{\varphi}_{n}^{\boldsymbol{a}}\right)_{Y_{\boldsymbol{a}}}:=\left(\tilde{u}, \tilde{\varphi}_{n}^{\boldsymbol{a}}\right)_{\overline{Y_{a}}}, \quad n \geqslant 1 .
$$

Note that (20) and (21) compute averages (with respective weights $\tilde{\phi}_{n}^{a}$ and $\tilde{\varphi}_{n}^{a}$ ) over: (i) a single wavelength in the $\boldsymbol{k}^{\boldsymbol{a}}$-direction, and (ii) dimension(s) of $Y$ in the direction(s) perpendicular to $\boldsymbol{k}^{\boldsymbol{a}}$.

Definition 2. Let $Y_{\mathfrak{m}}\left(\mathfrak{m}=\overline{1, \mathfrak{M}_{a}}\right)$ denote the contiguous open subregions of $Y_{\boldsymbol{a}}$ separated by an extended system of compliant interfaces $\Gamma_{\boldsymbol{a}}^{+}$, obtained by the $Y$-periodic replication of $\Gamma^{+}$over $Y_{\boldsymbol{a}}$. With reference to (8) and (21), we introduce the auxiliary (broken) Sobolev spaces

$$
\left.\begin{array}{rl}
H_{p}^{1}\left(Y_{\boldsymbol{a}}\right) & =\left\{g \in L^{2}\left(Y_{\boldsymbol{a}}\right): g \in L^{2}\left(Y_{\mathfrak{m}}\right), \nabla g \in\left(L^{2}\left(Y_{\mathfrak{m}}\right)\right)^{d},\left.1 g\right|_{\boldsymbol{a}, j} ^{-}=0 \text { on } \partial Y_{\boldsymbol{a}}, \mathfrak{m}=\overline{1, \mathfrak{M}_{\boldsymbol{a}}}\right\}, \\
H_{p 0}^{1 \boldsymbol{a}}\left(Y_{\boldsymbol{a}}\right) & =\left\{g \in H_{p}^{1}\left(Y_{\boldsymbol{a}}\right):\langle g\rangle_{\boldsymbol{a}}^{\varphi}=0\right\} .
\end{array}\right\}
$$

3.4. Scaling

Assuming that $\tilde{\lambda}_{n}^{a}$ is a simple eigenvalue, we describe the spectral neighborhood of $\left(\boldsymbol{k}^{\boldsymbol{a}}, \omega_{n}^{\boldsymbol{a}}\right)$ by scalings

$$
\boldsymbol{k}=\boldsymbol{k}^{\boldsymbol{a}}+\epsilon \hat{\boldsymbol{k}}, \quad \omega^{2}=\tilde{\lambda}_{n}^{a}+\epsilon^{2} \sigma \hat{\omega}^{2}, \quad \epsilon=o(1), \quad \sigma= \pm 1,
$$

see [16] for justification. By virtue of (6) and (23), $\tilde{u} \in H_{p}^{1}(Y)$ is found to satisfy

$$
\begin{gathered}
-\left(\tilde{\lambda}_{n}^{\boldsymbol{a}}+\epsilon^{2} \sigma \hat{\omega}^{2}\right) \rho \tilde{u}-\left(\nabla+i \boldsymbol{k}^{\boldsymbol{a}}+\epsilon i \hat{\boldsymbol{k}}\right) \cdot\left(G\left(\nabla+i \boldsymbol{k}^{\boldsymbol{a}}+\epsilon i \hat{\boldsymbol{k}}\right) \tilde{u}\right)=\tilde{f} \quad \text { in } Y, \\
\llbracket \tilde{u} \rrbracket+\kappa^{-1} t_{\boldsymbol{\nu}}^{\boldsymbol{k}^{\boldsymbol{a}}+\epsilon \hat{\boldsymbol{k}}}[\tilde{u}, \tilde{u}]=0 \quad \text { on } \Gamma,\left.\quad 1 t_{\boldsymbol{n}}^{\boldsymbol{k}^{\boldsymbol{a}}+\epsilon \hat{\boldsymbol{k}}}[\tilde{u}, \tilde{u}]\right|_{j} ^{+}=0 \quad \text { on } \partial Y .
\end{gathered}
$$

Remark 8. In the remainder of this work, we implicitly assume that $\|\tilde{f}\|_{L^{2}(Y)}=O(1)$ in situations when the source term is nont-trivial.

To aid the asymptotic analysis, we next introduce $\tilde{u} \in H_{p}^{1}\left(Y_{a}\right)$ as a field satisfying (24)-(25) over $Y_{\boldsymbol{a}}$ by the application of $Y$-periodicity, and we consider the factorized ansatz [16]

$$
\tilde{u}(\boldsymbol{x})=e^{-i \boldsymbol{k}^{\boldsymbol{a}} \cdot \boldsymbol{x}}\left(\epsilon^{-2} \tilde{u}_{0}(\boldsymbol{x})+\epsilon^{-1} \tilde{u}_{1}(\boldsymbol{x})+\tilde{u}_{2}(\boldsymbol{x})+\epsilon \tilde{u}_{3}(\boldsymbol{x})+\cdots\right),
$$

which distills (24)-(25) (self-repeated over $Y_{\boldsymbol{a}}$ ) into a series in $\epsilon$. On letting $\tilde{u}_{-1} \equiv 0$, we find from (25) and (26) that for every $m \geqslant 0, \tilde{u}_{m} \in H_{p}^{1}\left(Y_{\boldsymbol{a}}\right)$ are subject to the coupled boundary condition

$$
\left.1 t_{\boldsymbol{n}}^{\hat{\boldsymbol{k}}}\left[\tilde{u}_{m}, \tilde{u}_{m-1}\right]\right|_{\boldsymbol{a}, j} ^{+}=0 \quad \text { on } \partial Y_{\boldsymbol{a}} .
$$

In what follows, let $u_{m}$ denote the respective weighted averages of $\tilde{u}_{m}(\boldsymbol{x})$. Recalling (20), (21) and (26), we specifically seek $u_{m}$ as

$$
u_{m}=\left\langle\tilde{u}_{m}\right\rangle_{\boldsymbol{a}}^{\varphi}, \quad m \geqslant 0,
$$

so that

$$
\langle\tilde{u}\rangle_{\boldsymbol{a}}=\epsilon^{-2} u_{0}+\epsilon^{-1} u_{1}+u_{2}+\epsilon u_{3}+\ldots
$$


Notation. For brevity we denote by $\boldsymbol{I}$ the second-order identity tensor. In the sequel, we will also use $\{\cdot \boldsymbol{\cdot}$ to denote tensor averaging over all index permutations; in particular for an $m$ th-order tensor $\boldsymbol{g}$, we write

$$
\{\boldsymbol{g}\}_{j_{1}, j_{2}, \ldots j_{m}}=\frac{1}{m !} \sum_{\left(l_{1}, l_{2}, \ldots l_{m}\right) \in P} \boldsymbol{g}_{l_{1}, l_{2}, \ldots l_{m}}, \quad j_{1}, j_{2}, \ldots j_{m} \in \overline{1, d}
$$

where $P$ denotes the set of all permutations of $\left(j_{1}, j_{2}, \ldots j_{m}\right)$. Similarly, we will make use of the partial symmetrization

$$
\{\boldsymbol{g}\}_{j_{1}, j_{2}, \ldots j_{m}}^{\prime}=\frac{1}{(m-1) !} \sum_{\left(l_{2}, \ldots l_{m}\right) \in Q} \boldsymbol{g}_{j_{1}, l_{2}, \ldots l_{m}}, \quad j_{1}, j_{2}, \ldots j_{m} \in \overline{1, d}
$$

where $Q$ denotes the set of all permutations of $\left(j_{2}, j_{3}, \ldots j_{m}\right)$.

With the foregoing definitions, a tensorial variant of the conormal trace operator (7) can conveniently be introduced as

$$
\boldsymbol{t}_{\boldsymbol{\nu}}^{\boldsymbol{I}}[\boldsymbol{g}, \boldsymbol{h}](\boldsymbol{x})=\boldsymbol{\nu}(\boldsymbol{x}) \cdot G\left(\nabla \boldsymbol{g}(\boldsymbol{x})+\{\boldsymbol{I} \otimes \boldsymbol{h}(\boldsymbol{x})\}^{\prime}\right),
$$

where $\boldsymbol{g}$ and $\boldsymbol{h}$ are tensors of respective orders $m \geqslant 1$ and $l=m-1$, and $\nabla \boldsymbol{g}=\boldsymbol{e}_{j} \otimes \partial \boldsymbol{g} / \partial x_{j}$.

\subsection{Leading-order approximation}

From (24)-(27), one finds that $\tilde{u}_{0}(\boldsymbol{x})$ is governed by the $O\left(\epsilon^{-2}\right)$ system

$$
\begin{array}{r}
-\tilde{\lambda}_{n}^{a} \rho \tilde{u}_{0}-\nabla \cdot\left(G \nabla \tilde{u}_{0}\right)=0 \quad \text { in } Y_{\boldsymbol{a}}, \\
\llbracket \tilde{u}_{0} \rrbracket+\kappa^{-1} t_{\boldsymbol{\nu}}\left[\tilde{u}_{0}\right]=0 \quad \text { on } \Gamma_{\boldsymbol{a}},\left.\quad 1 t_{\boldsymbol{n}}\left[\tilde{u}_{0}\right]\right|_{\boldsymbol{a}, j} ^{+}=0
\end{array}
$$

A comparison with (18) reveals that

$$
\tilde{u}_{0}(\boldsymbol{x})=u_{0} \tilde{\varphi}_{n}^{\boldsymbol{a}}(\boldsymbol{x}),
$$

where $u_{0}$ is a constant to be determined.

In a similar fashion, the $O\left(\epsilon^{-1}\right)$ system governing $\tilde{u}_{1} \in H_{p}^{1}\left(Y_{\boldsymbol{a}}\right)$ can be identified as

$$
\begin{gathered}
-\tilde{\lambda}_{n}^{\boldsymbol{a}} \rho \tilde{u}_{1}-\nabla \cdot\left(G\left(\nabla \tilde{u}_{1}+i \hat{\boldsymbol{k}} \tilde{u}_{0}\right)\right)-i \hat{\boldsymbol{k}} \cdot\left(G \nabla \tilde{u}_{0}\right)=0 \quad \text { in } Y_{\boldsymbol{a}}, \\
\llbracket \tilde{u}_{1} \rrbracket+\kappa^{-1} t_{\boldsymbol{\nu}}^{\hat{\boldsymbol{k}}}\left[\tilde{u}_{1}, \tilde{u}_{0}\right]=0 \quad \text { on } \Gamma_{\boldsymbol{a}},\left.\quad 1 t_{\boldsymbol{n}}^{\hat{\boldsymbol{k}}}\left[\tilde{u}_{1}, \tilde{u}_{0}\right]\right|_{\boldsymbol{a}, j} ^{+}=0 \quad \text { on } \partial Y_{\boldsymbol{a}} .
\end{gathered}
$$

By the linearity of the problem, we find that

$$
\tilde{u}_{1}(\boldsymbol{x})=u_{0} \chi^{(1)}(\boldsymbol{x}) \cdot i \hat{\boldsymbol{k}}+u_{1} \tilde{\varphi}_{n}^{\boldsymbol{a}}(\boldsymbol{x}),
$$

where $u_{1}$ is a constant, and $\chi^{(1)} \in\left(H_{p 0}^{1 a}\left(Y_{\boldsymbol{a}}\right)\right)^{d}$ is a vector that satisfies

$$
\begin{gathered}
\tilde{\lambda}_{n}^{\boldsymbol{a}} \rho \boldsymbol{\chi}^{(1)}+\nabla \cdot\left(G\left(\nabla \boldsymbol{\chi}^{(1)}+\boldsymbol{I} \tilde{\varphi}_{n}^{\boldsymbol{a}}\right)\right)+G \nabla \tilde{\varphi}_{n}^{\boldsymbol{a}}=\mathbf{0} \quad \text { in } Y_{\boldsymbol{a}}, \\
\llbracket \boldsymbol{\chi}^{(1)} \rrbracket+\kappa^{-1} \boldsymbol{t}_{\boldsymbol{\nu}}^{\boldsymbol{I}}\left[\boldsymbol{\chi}^{(1)}, \tilde{\varphi}_{n}^{\boldsymbol{a}}\right]=\mathbf{0} \quad \text { on } \Gamma_{\boldsymbol{a}}, \quad\left|\boldsymbol{t}_{\boldsymbol{n}}^{\boldsymbol{I}}\left[\boldsymbol{\chi}^{(1)}, \tilde{\varphi}_{n}^{\boldsymbol{a}}\right]\right|_{\boldsymbol{a}, j}^{+}=\mathbf{0} \quad \text { on } \partial Y_{\boldsymbol{a}} .
\end{gathered}
$$

Lemma 2. The "unit" cell problem (33)-(34) has a unique real-valued solution in $\left(H_{p 0}^{1 a}\left(Y_{\boldsymbol{a}}\right)\right)^{d}$. See Appendix, Section 7 for proof.

Proceeding with the cascade of boundary value problems, we find the $O(1)$ contribution to $(24)-(25)$ as

$$
\begin{gathered}
-\tilde{\lambda}_{n}^{\boldsymbol{a}} \rho \tilde{u}_{2}-\nabla \cdot\left(G\left(\nabla \tilde{u}_{2}+i \hat{\boldsymbol{k}} \tilde{u}_{1}\right)\right)-i \hat{\boldsymbol{k}} \cdot\left(G\left(\nabla \tilde{u}_{1}+i \hat{\boldsymbol{k}} \tilde{u}_{0}\right)\right)-\sigma \hat{\omega}^{2} \rho \tilde{u}_{0}=\tilde{f} e^{i \boldsymbol{k}^{\boldsymbol{a}} \cdot \boldsymbol{x}} \quad \text { in } Y_{\boldsymbol{a}}, \\
\llbracket \tilde{u}_{2} \rrbracket+\kappa^{-1} t_{\boldsymbol{\nu}}^{\hat{\boldsymbol{k}}}\left[\tilde{u}_{2}, \tilde{u}_{1}\right]=0 \quad \text { on } \Gamma_{\boldsymbol{a}},\left.\quad 1 t_{\boldsymbol{n}}^{\hat{\boldsymbol{k}}}\left[\tilde{u}_{2}, \tilde{u}_{1}\right]\right|_{\boldsymbol{a}, j} ^{+}=0 \quad \text { on } \partial Y_{\boldsymbol{a}} .
\end{gathered}
$$

To isolate the equation governing $u_{0}$, one may evaluate the weighted average $\langle(35)\rangle_{\boldsymbol{a}}^{\boldsymbol{\varphi}}$, namely

$$
\left(-\tilde{\lambda}_{n}^{\boldsymbol{a}} \rho \tilde{u}_{2}-\nabla \cdot\left(G\left(\nabla \tilde{u}_{2}+i \hat{\boldsymbol{k}} \tilde{u}_{1}\right)\right), \tilde{\varphi}_{n}^{\boldsymbol{a}}\right)_{\overline{Y_{\boldsymbol{a}}}}=\left(i \hat{\boldsymbol{k}} \cdot G\left(\nabla \tilde{u}_{1}+i \hat{\boldsymbol{k}} \tilde{u}_{0}\right), \tilde{\varphi}_{n}^{\boldsymbol{a}}\right)_{\bar{Y}_{\boldsymbol{a}}}+\sigma \hat{\omega}^{2}\left\langle\rho \tilde{\varphi}_{n}^{\boldsymbol{a}}\right\rangle_{\boldsymbol{a}}^{\varphi} u_{0}+\left\langle\tilde{f} e^{i \boldsymbol{k}^{\boldsymbol{a}} \cdot \boldsymbol{x}}\right\rangle_{\boldsymbol{a}}^{\varphi} .
$$


On recalling (18) and integrating by parts, the second term on the left-hand side of (37) reduces as

$$
\begin{aligned}
& -\left(\nabla \cdot\left(G\left(\nabla \tilde{u}_{2}+i \hat{\boldsymbol{k}} \tilde{u}_{1}\right)\right), \tilde{\varphi}_{n}^{\boldsymbol{a}}\right)_{\overline{Y_{\boldsymbol{a}}}}=\left(G\left(\nabla \tilde{u}_{2}+i \hat{\boldsymbol{k}} \tilde{u}_{1}\right), \nabla \tilde{\varphi}_{n}^{\boldsymbol{a}}\right)_{\bar{Y}_{\boldsymbol{a}}}+\left|Y_{\boldsymbol{a}}\right|^{-1}\left(t_{\boldsymbol{\nu}}^{\hat{\boldsymbol{k}}}\left[\tilde{u}_{2}, \tilde{u}_{1}\right], \llbracket \tilde{\varphi}_{n}^{\boldsymbol{a}} \rrbracket\right)_{\Gamma_{\boldsymbol{a}}} \\
& =-\left(\tilde{u}_{2}, \nabla \cdot\left(G \nabla \tilde{\varphi}_{n}^{\boldsymbol{a}}\right)\right)_{\bar{Y}_{\boldsymbol{a}}}+\left(i \hat{\boldsymbol{k}} G \tilde{u}_{1}, \nabla \tilde{\varphi}_{n}^{\boldsymbol{a}}\right)_{\overline{Y_{\boldsymbol{a}}}}+\left|Y_{\boldsymbol{a}}\right|^{-1}\left(t_{\boldsymbol{\nu}}^{\hat{k}}\left[\tilde{u}_{2}, \tilde{u}_{1}\right], \llbracket \tilde{\varphi}_{n}^{\boldsymbol{a}} \rrbracket\right)_{\Gamma_{\boldsymbol{a}}}-\left|Y_{\boldsymbol{a}}\right|^{-1}\left(\llbracket \tilde{u}_{2} \rrbracket, t_{\boldsymbol{\nu}}\left[\tilde{\varphi}_{n}^{\boldsymbol{a}}\right]\right)_{\Gamma_{\boldsymbol{a}}} \\
& =\left(\tilde{u}_{2}, \tilde{\lambda}_{n}^{\boldsymbol{a}} \rho \tilde{\varphi}_{n}^{\boldsymbol{a}}\right)_{\bar{Y}_{\boldsymbol{a}}}+\left(i \hat{\boldsymbol{k}} G \tilde{u}_{1}, \nabla \tilde{\varphi}_{n}^{\boldsymbol{a}}\right)_{\overline{Y_{\boldsymbol{a}}}},
\end{aligned}
$$

thanks to the use of boundary and interfacial conditions (19) and (36). By (32) and (38), the left-hand side of (37) becomes

$$
\left(i \hat{\boldsymbol{k}} G \tilde{u}_{1}, \nabla \tilde{\varphi}_{n}^{\boldsymbol{a}}\right)_{\bar{Y}_{\boldsymbol{a}}}=u_{0}\left(G \boldsymbol{\chi}^{(1)} \otimes \nabla \tilde{\varphi}_{n}^{\boldsymbol{a}}, 1\right)_{\overline{Y_{\boldsymbol{a}}}}:(i \hat{\boldsymbol{k}})^{2}+u_{1}\left(i \hat{\boldsymbol{k}} G \tilde{\varphi}_{n}^{\boldsymbol{a}}, \nabla \tilde{\varphi}_{n}^{\boldsymbol{a}}\right)_{\overline{Y_{\boldsymbol{a}}}},
$$

while its right-hand side reads

$$
u_{0}\left(G \nabla \boldsymbol{\chi}^{(1)}, \tilde{\varphi}_{n}^{\boldsymbol{a}}\right)_{\overline{Y_{\boldsymbol{a}}}}:(i \hat{\boldsymbol{k}})^{2}+u_{1}\left(i \hat{\boldsymbol{k}} \cdot G \nabla \tilde{\varphi}_{n}^{\boldsymbol{a}}, \tilde{\varphi}_{n}^{\boldsymbol{a}}\right)_{\bar{Y}_{\boldsymbol{a}}}+u_{0}\left(G \boldsymbol{I} \tilde{\varphi}_{n}^{\boldsymbol{a}}, \tilde{\varphi}_{n}^{\boldsymbol{a}}\right)_{\overline{Y_{a}}}:(i \hat{\boldsymbol{k}})^{2}+\sigma \hat{\omega}^{2}\left\langle\rho \tilde{\varphi}_{n}^{\boldsymbol{a}}\right\rangle_{\boldsymbol{a}}^{\varphi} u_{0}+\left\langle\tilde{f} e^{i \boldsymbol{k}^{\boldsymbol{a}} \cdot \boldsymbol{x}}\right\rangle_{\boldsymbol{a}}^{\varphi}
$$

From the last two results and the fact that $\tilde{\varphi}_{n}^{a}$ is real-valued, we find the effective equation for $u_{0}$

$$
-\left(\boldsymbol{\mu}^{(0)}:(i \hat{\boldsymbol{k}})^{2}+\sigma \rho^{(0)} \hat{\omega}^{2}\right) u_{0}=\left\langle\tilde{f} e^{i \boldsymbol{k}^{a} \cdot \boldsymbol{x}}\right\rangle_{\boldsymbol{a}}^{\varphi},
$$

featuring the effective coefficients

$$
\rho^{(0)}=\left\langle\rho \tilde{\varphi}_{n}^{\boldsymbol{a}}\right\rangle_{\boldsymbol{a}}^{\varphi}, \quad \boldsymbol{\mu}^{(0)}=\left\langle G\left\{\nabla \boldsymbol{\chi}^{(1)}+\boldsymbol{I} \tilde{\varphi}_{n}^{\boldsymbol{a}}\right\}\right\rangle_{\boldsymbol{a}}^{\varphi}-\left(G\left\{\boldsymbol{\chi}^{(1)} \otimes \nabla \tilde{\varphi}_{n}^{a}\right\}, 1\right)_{\overline{Y_{\boldsymbol{a}}}}
$$

that are real-valued by Lemma 1 and Lemma 2. We note that (i) $\rho^{(0)} \neq \rho$ in general due to Remark 3, and (ii) the symmetrized expression for $\boldsymbol{\mu}^{(0)}$ (according to (28)) is due to the structure of $\boldsymbol{\mu}^{(0)}:(i \hat{\boldsymbol{k}})^{2}$, which is invariant with respect to the index permutation of $\boldsymbol{\mu}^{(0)}$.

It is perhaps not surprising to observe that the effective equation (39) and its coefficients carry the same general structure as those describing waves in periodic continua [16], with differences being confined to the definition of the eigenfunction $\tilde{\varphi}_{n}^{a}$ and the first cell function $\chi^{(1)}$, which are in the present case discontinuous across compliant interfaces.

\subsection{First-order corrector}

To expose the field equation governing $u_{1}$, we introduce $\boldsymbol{\chi}^{(2)} \in\left(H_{p 0}^{1 \boldsymbol{a}}\left(Y_{\boldsymbol{a}}\right)\right)^{d \times d}$ as the unique second-order tensor solving

$$
\begin{gathered}
\tilde{\lambda}_{n}^{\boldsymbol{a}} \rho \boldsymbol{\chi}^{(2)}+\nabla \cdot\left(G\left(\nabla \boldsymbol{\chi}^{(2)}+\left\{\boldsymbol{I} \otimes \boldsymbol{\chi}^{(1)}\right\}^{\prime}\right)\right)+G\left\{\nabla \boldsymbol{\chi}^{(1)}+\boldsymbol{I} \tilde{\varphi}_{n}^{\boldsymbol{a}}\right\}-\frac{\rho}{\rho^{(0)}} \boldsymbol{\mu}^{(0)} \tilde{\varphi}_{n}^{\boldsymbol{a}}=\mathbf{0} \quad \text { in } Y_{\boldsymbol{a}}, \\
\llbracket \boldsymbol{\chi}^{(2)} \rrbracket+\kappa^{-1} \boldsymbol{t}_{\boldsymbol{\nu}}^{I}\left[\boldsymbol{\chi}^{(2)}, \boldsymbol{\chi}^{(1)}\right]=\mathbf{0} \quad \text { on } \Gamma_{\boldsymbol{a}},\left.\quad 1 \boldsymbol{t}_{\boldsymbol{n}}^{I}\left[\boldsymbol{\chi}^{(2)}, \boldsymbol{\chi}^{(1)}\right]\right|_{\boldsymbol{a}, j} ^{+}=\mathbf{0} \quad \text { on } \partial Y_{\boldsymbol{a}} .
\end{gathered}
$$

and we let $\eta^{(0)} \in H_{p 0}^{1 \boldsymbol{a}}\left(Y_{\boldsymbol{a}}\right)$ be the unique function satisfying

$$
\begin{array}{ccc}
\tilde{\lambda}_{n}^{\boldsymbol{a}} \rho \eta^{(0)}+\nabla \cdot\left(G \nabla \eta^{(0)}\right)-\frac{\rho}{\rho^{(0)}}\left\langle\tilde{f} e^{i \boldsymbol{k}^{\boldsymbol{a}} \cdot \boldsymbol{x}}\right\rangle_{\boldsymbol{a}}^{\varphi} \tilde{\varphi}_{n}^{\boldsymbol{a}}+\tilde{f} e^{i \boldsymbol{k}^{\boldsymbol{a}} \cdot \boldsymbol{x}}=0 & \text { in } Y_{\boldsymbol{a}}, \\
\llbracket \eta^{(0)} \rrbracket+\kappa^{-1} t_{\boldsymbol{\nu}}\left[\eta^{(0)}\right]=0 \quad \text { on } \Gamma_{\boldsymbol{a}},\left.\quad 1 t_{\boldsymbol{n}}\left[\eta^{(0)}\right]\right|_{\boldsymbol{a}, j} ^{+}=0 \quad \text { on } \partial Y_{\boldsymbol{a}},
\end{array}
$$

see Remark 12. With such definitions, one can show that (35)-(36) is solved by

$$
\tilde{u}_{2}(\boldsymbol{x})=u_{0} \boldsymbol{\chi}^{(2)}(\boldsymbol{x}):(i \hat{\boldsymbol{k}})^{2}+u_{1} \chi^{(1)}(\boldsymbol{x}) \cdot i \hat{\boldsymbol{k}}+u_{2} \tilde{\varphi}_{n}^{\boldsymbol{a}}(\boldsymbol{x})+\eta^{(0)}(\boldsymbol{x}) .
$$

Lemma 3. The cell function $\eta^{(0)}$ verifies the identity

$$
\left\langle G \nabla \eta^{(0)}\right\rangle_{\boldsymbol{a}}^{\varphi}-\left(G \eta^{(0)}, \nabla \tilde{\varphi}_{n}^{\boldsymbol{a}}\right)_{\overline{Y_{a}}}=\frac{\left\langle\tilde{f} e^{i \boldsymbol{k}^{\boldsymbol{a}} \cdot \boldsymbol{x}}\right\rangle_{\boldsymbol{a}}^{\varphi}}{\rho^{(0)}} \boldsymbol{\rho}^{(1)}-\left(\tilde{f} e^{i \boldsymbol{k}^{a} \cdot \boldsymbol{x}}, \chi^{(1)}\right)_{\overline{Y_{a}}},
$$

where $\boldsymbol{\rho}^{(1)}=\left\langle\rho \boldsymbol{\chi}^{(1)}\right\rangle_{\boldsymbol{a}}^{\varphi}$. See Appendix, Section 7 for proof. 
Proceeding with the asymptotic analysis, the $O(\epsilon)$ problem stemming from (24)-(25) reads

$$
\begin{gathered}
-\tilde{\lambda}_{n}^{\boldsymbol{a}} \rho \tilde{u}_{3}-\nabla \cdot\left(G\left(\nabla \tilde{u}_{3}+i \hat{\boldsymbol{k}} \tilde{u}_{2}\right)\right)-i \hat{\boldsymbol{k}} \cdot\left(G\left(\nabla \tilde{u}_{2}+i \hat{\boldsymbol{k}} \tilde{u}_{1}\right)\right)-\sigma \hat{\omega}^{2} \rho \tilde{u}_{1}=0 \quad \text { in } Y_{\boldsymbol{a}}, \\
\llbracket \tilde{u}_{3} \rrbracket+\kappa^{-1} t_{\boldsymbol{\nu}}^{\hat{\boldsymbol{k}}}\left[\tilde{u}_{3}, \tilde{u}_{2}\right]=0 \quad \text { on } \Gamma_{\boldsymbol{a}},\left.\quad 1 t_{\boldsymbol{n}}^{\hat{\boldsymbol{k}}}\left[\tilde{u}_{3}, \tilde{u}_{2}\right]\right|_{\boldsymbol{a}, j} ^{+}=0 \quad \text { on } \partial Y_{\boldsymbol{a}} .
\end{gathered}
$$

To expose the behavior of $u_{1}$, it is instructive to compute the weighted average (21) of (47), i.e.

$$
\left(-\tilde{\lambda}_{n}^{\boldsymbol{a}} \rho \tilde{u}_{3}-\nabla \cdot\left(G\left(\nabla \tilde{u}_{3}+i \hat{\boldsymbol{k}} \tilde{u}_{2}\right)\right), \tilde{\varphi}_{n}^{\boldsymbol{a}}\right)_{\overline{Y_{\boldsymbol{a}}}}=\left(i \hat{\boldsymbol{k}} \cdot\left(G\left(\nabla \tilde{u}_{2}+i \hat{\boldsymbol{k}} \tilde{u}_{1}\right)\right), \tilde{\varphi}_{n}^{\boldsymbol{a}}\right)_{\overline{Y_{\boldsymbol{a}}}}+\sigma \hat{\omega}^{2}\left\langle\rho \tilde{u}_{1}\right\rangle_{\boldsymbol{a}}^{\varphi} .
$$

Integrating (49) by parts "a la" the treatment of (35) and making use of (32), (36), (48), (45) and Lemma 3 , we find that

$$
-\left(\boldsymbol{\mu}^{(0)}:(i \hat{\boldsymbol{k}})^{2}+\sigma \rho^{(0)} \hat{\omega}^{2}\right) u_{1}-\left(\boldsymbol{\mu}^{(1)}:(i \hat{\boldsymbol{k}})^{3}+\sigma \boldsymbol{\rho}^{(1)} \cdot i \hat{\boldsymbol{k}} \hat{\omega}^{2}\right) u_{0}=\left(\frac{\left\langle\tilde{f} e^{i \boldsymbol{k}^{a} \cdot \boldsymbol{x}}\right\rangle_{a}^{\varphi}}{\rho^{(0)}} \boldsymbol{\rho}^{(1)}-\left(\tilde{f} e^{i \boldsymbol{k}^{\boldsymbol{a}} \cdot \boldsymbol{x}}, \boldsymbol{\chi}^{(1)}\right)_{\overline{Y_{a}}}\right) \cdot i \hat{\boldsymbol{k}}
$$

where

$$
\boldsymbol{\rho}^{(1)}=\left\langle\rho \boldsymbol{\chi}^{(1)}\right\rangle_{\boldsymbol{a}}^{\varphi}, \quad \boldsymbol{\mu}^{(1)}=\left\langle G\left\{\nabla \boldsymbol{\chi}^{(2)}+\boldsymbol{I} \otimes \boldsymbol{\chi}^{(1)}\right\}\right\rangle_{\boldsymbol{a}}^{\varphi}-\left(G\left\{\boldsymbol{\chi}^{(2)} \otimes \nabla \tilde{\varphi}_{n}^{\boldsymbol{a}}\right\}, 1\right)_{\bar{Y}_{\boldsymbol{a}}}
$$

Remark 9. Due to the fact that $\boldsymbol{\chi}^{(1)} \in \mathbb{R}^{d}$, one finds by following the proof of Lemma 2 that $\boldsymbol{\chi}^{(2)} \in \mathbb{R}^{d \times d}$. As a result, $\boldsymbol{\rho}^{(1)} \in \mathbb{R}^{d}$ and $\boldsymbol{\mu}^{(1)} \in \mathbb{R}^{d \times d \times d}$.

Lemma 4. The effective tensorial coefficients in (51) have the property

$$
\rho^{(0)} \boldsymbol{\mu}^{(1)}=\left\{\boldsymbol{\rho}^{(1)} \otimes \boldsymbol{\mu}^{(0)}\right\},
$$

by which (50) reduces to

$$
-\left(\boldsymbol{\mu}^{(0)}:(i \hat{\boldsymbol{k}})^{2}+\sigma \rho^{(0)} \hat{\omega}^{2}\right) u_{1}=-\left(\chi^{(1)} \tilde{f} e^{i \boldsymbol{k}^{\boldsymbol{a}} \cdot \boldsymbol{x}}, 1\right)_{\overline{Y_{a}}} \cdot i \hat{\boldsymbol{k}}
$$

See Appendix, Section 7 for proof.

\subsection{Second-order corrector}

We next introduce $\chi^{(3)} \in\left(H_{p 0}^{1 \boldsymbol{a}}\left(Y_{\boldsymbol{a}}\right)\right)^{d \times d \times d}$ as the unique "zero-mean" third-order tensor solving

$$
\begin{gathered}
\tilde{\lambda}_{n}^{\boldsymbol{a}} \rho \boldsymbol{\chi}^{(3)}+\nabla \cdot\left(G\left(\nabla \boldsymbol{\chi}^{(3)}+\left\{\boldsymbol{I} \otimes \boldsymbol{\chi}^{(2)}\right\}^{\prime}\right)\right)+G\left\{\nabla \boldsymbol{\chi}^{(2)}+\boldsymbol{I} \otimes \boldsymbol{\chi}^{(1)}\right\}-\frac{\rho}{\rho^{(0)}}\left\{\boldsymbol{\mu}^{(0)} \otimes \boldsymbol{\chi}^{(1)}\right\}=\mathbf{0} \quad \text { in } Y_{\boldsymbol{a}}, \\
\llbracket \boldsymbol{\chi}^{(3)} \rrbracket+\kappa^{-1} \boldsymbol{t}_{\boldsymbol{\nu}}^{\boldsymbol{I}}\left[\boldsymbol{\chi}^{(3)}, \boldsymbol{\chi}^{(2)}\right]=\mathbf{0} \quad \text { on } \Gamma_{\boldsymbol{a}},\left.\quad 1 \boldsymbol{t}_{\boldsymbol{n}}^{\boldsymbol{I}}\left[\boldsymbol{\chi}^{(3)}, \boldsymbol{\chi}^{(2)}\right]\right|_{\boldsymbol{a}, j} ^{+}=\mathbf{0} \quad \text { on } \partial Y_{\boldsymbol{a}} .
\end{gathered}
$$

and we let $\boldsymbol{\eta}^{(1)} \in\left(H_{p 0}^{1 a}\left(Y_{\boldsymbol{a}}\right)\right)^{d}$ be the unique "zero-mean" vector solving

$$
\begin{gathered}
\tilde{\lambda}_{n}^{\boldsymbol{a}} \rho \boldsymbol{\eta}^{(1)}+\nabla \cdot\left(G\left(\nabla \boldsymbol{\eta}^{(1)}+\boldsymbol{I} \eta^{(0)}\right)\right)+G \nabla \eta^{(0)}+\frac{\rho}{\rho^{(0)}}\left(\left(\chi^{(1)} \tilde{f} e^{i \boldsymbol{k}^{\boldsymbol{a}} \cdot \boldsymbol{x}}, 1\right)_{\bar{Y}_{\boldsymbol{a}}} \tilde{\varphi}_{n}^{\boldsymbol{a}}-\left\langle\tilde{f} e^{i \boldsymbol{k}^{\boldsymbol{a}} \cdot \boldsymbol{x}}\right\rangle_{\boldsymbol{a}}^{\varphi} \chi^{(1)}\right)=\mathbf{0} \quad \text { in } Y_{\boldsymbol{a}}, \\
\llbracket \boldsymbol{\eta}^{(1)} \rrbracket+\kappa^{-1} \boldsymbol{t}_{\boldsymbol{\nu}}^{I}\left[\boldsymbol{\eta}^{(1)}, \eta^{(0)}\right]=\mathbf{0} \quad \text { on } \Gamma_{\boldsymbol{a}},\left.\quad 1 \boldsymbol{t}_{\boldsymbol{n}}^{\boldsymbol{I}}\left[\boldsymbol{\eta}^{(1)}, \eta^{(0)}\right]\right|_{\boldsymbol{a}, j} ^{+}=\mathbf{0} \quad \text { on } \partial Y_{\boldsymbol{a}} .
\end{gathered}
$$

With such definitions, it can be shown that

$$
\tilde{u}_{3}(\boldsymbol{x})=u_{0} \boldsymbol{\chi}^{(3)}(\boldsymbol{x}):(i \hat{\boldsymbol{k}})^{3}+u_{1} \boldsymbol{\chi}^{(2)}(\boldsymbol{x}):(i \hat{\boldsymbol{k}})^{2}+u_{2} \boldsymbol{\chi}^{(1)}(\boldsymbol{x}) \cdot i \hat{\boldsymbol{k}}+u_{3} \tilde{\varphi}_{n}^{\boldsymbol{a}}(\boldsymbol{x})+\boldsymbol{\eta}^{(1)}(\boldsymbol{x}) \cdot i \hat{\boldsymbol{k}}
$$

solves $(47)-(48)$. To isolate the behavior of the constant $u_{2}$, we finally consider the $O\left(\epsilon^{2}\right)$ contribution to $(24)-$ (25), namely

$$
\begin{gathered}
-\tilde{\lambda}_{n}^{\boldsymbol{a}} \rho \tilde{u}_{4}-\nabla \cdot\left(G\left(\nabla \tilde{u}_{4}+i \hat{\boldsymbol{k}} \tilde{u}_{3}\right)\right)-i \hat{\boldsymbol{k}} \cdot\left(G\left(\nabla \tilde{u}_{3}+i \hat{\boldsymbol{k}} \tilde{u}_{2}\right)\right)-\sigma \hat{\omega}^{2} \rho \tilde{u}_{2}=0 \quad \text { in } Y_{\boldsymbol{a}}, \\
\llbracket \tilde{u}_{4} \rrbracket+\kappa^{-1} t_{\boldsymbol{\nu}}^{\hat{\boldsymbol{k}}}\left[\tilde{u}_{4}, \tilde{u}_{3}\right]=0 \quad \text { on } \Gamma_{\boldsymbol{a}},\left.\quad 1 t_{\boldsymbol{n}}^{\hat{\boldsymbol{k}}}\left[\tilde{u}_{4}, \tilde{u}_{3}\right]\right|_{\boldsymbol{a}, j} ^{+}=0 \quad \text { on } \partial Y_{\boldsymbol{a}} .
\end{gathered}
$$

Integrating by parts the weighed equality $\left((55), \tilde{\varphi}_{n}^{a}\right)_{Y_{a}}$ as in the treatment of (47), we obtain

$$
\begin{aligned}
-\left(\boldsymbol{\mu}^{(0)}:(i \hat{\boldsymbol{k}})^{2}+\right. & \left.\sigma \rho^{(0)} \hat{\omega}^{2}\right) u_{2}-\left(\boldsymbol{\mu}^{(2)}:(i \hat{\boldsymbol{k}})^{4}+\sigma \boldsymbol{\rho}^{(2)}:(i \hat{\boldsymbol{k}})^{2} \hat{\omega}^{2}\right) u_{0}=\sigma\left\langle\rho \eta^{(0)}\right\rangle_{\boldsymbol{a}}^{\varphi} \hat{\omega}^{2} \\
& +\left(\left\langle G\left\{\nabla \boldsymbol{\eta}^{(1)}+\boldsymbol{I} \eta^{(0)}\right\}\right\rangle_{\boldsymbol{a}}^{\varphi}-\left(G\left\{\boldsymbol{\eta}^{(1)} \otimes \nabla \tilde{\varphi}_{n}^{\boldsymbol{a}}\right\}, 1\right)_{\bar{Y}_{\boldsymbol{a}}}+\frac{1}{\rho^{(0)}}\left\{\boldsymbol{\rho}^{(1)} \otimes\left(\boldsymbol{\chi}^{(1)} \tilde{f} e^{i \boldsymbol{k}^{\boldsymbol{a}} \cdot \boldsymbol{x}}, 1\right)_{\overline{Y_{a}}}\right\}\right):(i \hat{\boldsymbol{k}})^{2},
\end{aligned}
$$

where

$$
\boldsymbol{\rho}^{(2)}=\left\langle\rho \boldsymbol{\chi}^{(2)}\right\rangle_{\boldsymbol{a}}^{\varphi}, \quad \boldsymbol{\mu}^{(2)}=\left\langle G\left\{\nabla \boldsymbol{\chi}^{(3)}+\boldsymbol{I} \otimes \boldsymbol{\chi}^{(2)}\right\}\right\rangle_{\boldsymbol{a}}^{\varphi}-\left(G\left\{\boldsymbol{\chi}^{(3)} \otimes \nabla \tilde{\varphi}_{n}^{\boldsymbol{a}}\right\}, 1\right)_{\overline{Y_{\boldsymbol{a}}}} .
$$




\subsection{Second-order FW-FF approximation}

The following theorem summarizes the asymptotic result stemming from the preceding analysis. This result is formal, as it does not include remainder estimates. The predicted behavior for small $\epsilon$ is supported by computational evidence, see Section 6.

Theorem 1. Assume that the Bloch wave function $\tilde{u}$ solves (6) with the source term $\tilde{f}$, and consider the effective wave motion $\langle\tilde{u}\rangle_{\boldsymbol{a}}$ according to (20) near apex $\boldsymbol{k}^{\boldsymbol{a}}$ of the first "quadrant" (13) of the first Brillouin zone $\mathcal{B}^{+}$. Provided that the eigenvalue $\tilde{\lambda}_{n}^{\boldsymbol{a}}(n \geqslant 1)$ solving (18) is simple, the second-order $F W$-FF approximation of $\langle\tilde{u}\rangle_{\boldsymbol{a}}$ in a neighborhood of $\left(\boldsymbol{k}^{\boldsymbol{a}}, \omega_{n}^{\boldsymbol{a}}\right)$ satisfies

$$
-\left(\boldsymbol{\mu}^{(0)}:(i \hat{\boldsymbol{k}})^{2}+\sigma \rho^{(0)} \hat{\omega}^{2}\right)\langle\tilde{u}\rangle_{\boldsymbol{a}}-\epsilon^{2}\left(\boldsymbol{\mu}^{(2)}:(i \hat{\boldsymbol{k}})^{4}+\sigma \boldsymbol{\rho}^{(2)}:(i \hat{\boldsymbol{k}})^{2} \hat{\omega}^{2}\right)\langle\tilde{u}\rangle_{\boldsymbol{a}} \stackrel{\epsilon}{=} \epsilon^{-2} F(\hat{\boldsymbol{k}}, \hat{\omega}, \epsilon),
$$

where "트" signifies equality with an $O(\epsilon)$ residual; the coefficients of homogenization $\rho^{(0)} \in \mathbb{R}, \boldsymbol{\mu}^{(0)} \in \mathbb{R}^{d \times d}, \boldsymbol{\rho}^{(2)} \in$ $\mathbb{R}^{d \times d}$ and $\boldsymbol{\mu}^{(2)} \in \mathbb{R}^{d \times d \times d \times d}$ are given by (40) and (56); and

$$
\begin{aligned}
F(\hat{\boldsymbol{k}}, \hat{\omega}, \epsilon)= & \left\langle\tilde{f} e^{i \boldsymbol{k}^{a} \cdot \boldsymbol{x}}\right\rangle_{\boldsymbol{a}}^{\varphi}-\epsilon\left(\boldsymbol{\chi}^{(1)} \tilde{f} e^{i \boldsymbol{k}^{\boldsymbol{a}} \cdot \boldsymbol{x}}, 1\right)_{\overline{Y_{a}}} \cdot i \hat{\boldsymbol{k}}+\epsilon^{2}\left\langle\rho \eta^{(0)}\right\rangle_{\boldsymbol{a}}^{\varphi} \sigma \hat{\omega}^{2} \\
& +\epsilon^{2}\left(\left\langle G\left\{\nabla \boldsymbol{\eta}^{(1)}+\boldsymbol{I} \eta^{(0)}\right\}\right\rangle_{\boldsymbol{a}}^{\varphi}-\left(G\left\{\boldsymbol{\eta}^{(1)} \otimes \nabla \tilde{\varphi}_{n}^{\boldsymbol{a}}\right\}, 1\right)_{\bar{Y}_{\boldsymbol{a}}}+\frac{1}{\rho^{(0)}}\left\{\boldsymbol{\rho}^{(1)} \otimes\left(\boldsymbol{\chi}^{(1)} \tilde{f} e^{i \boldsymbol{k}^{\boldsymbol{a}} \cdot \boldsymbol{x}}, 1\right)_{\overline{Y_{a}}}\right\}\right):(i \hat{\boldsymbol{k}})^{2} .
\end{aligned}
$$

In the context of source-free wave motion $(\tilde{f}=0)$, the second-order $F W$-FF approximation of the $n$th dispersion branch near $\boldsymbol{k}=\boldsymbol{k}^{\boldsymbol{a}}$ accordingly reads

$$
\tilde{\lambda}_{n}^{\boldsymbol{a}}(\epsilon)-\tilde{\lambda}_{n}^{\boldsymbol{a}} \stackrel{\epsilon^{3}}{=} \frac{\epsilon^{2} \boldsymbol{\mu}^{(0)}: \hat{\boldsymbol{k}}^{2}-\epsilon^{4} \boldsymbol{\mu}^{(2)}: \hat{\boldsymbol{k}}^{4}}{\rho^{(0)}-\epsilon^{2} \boldsymbol{\rho}^{(2)}: \hat{\boldsymbol{k}}^{2}},
$$

where $\tilde{\lambda}_{n}^{\boldsymbol{a}}(\epsilon):=\tilde{\lambda}_{n}\left(\boldsymbol{k}^{\boldsymbol{a}}+\epsilon \hat{\boldsymbol{k}}\right)$.

Remark 10. Using the second-order approximation of $\tilde{\lambda}_{n}^{a}(\epsilon)$ provided by Theorem 1, the affiliated approximation of $\langle\tilde{u}\rangle_{\boldsymbol{a}}$ can be written as

$$
\langle\tilde{u}\rangle_{\boldsymbol{a}} \stackrel{\epsilon}{=} \frac{F(\hat{\boldsymbol{k}}, \hat{\omega}, \epsilon)}{\rho^{(0)}-\epsilon^{2} \boldsymbol{\rho}^{(2)}: \hat{\boldsymbol{k}}^{2}} \frac{1}{\tilde{\lambda}_{n}^{\boldsymbol{a}}(\epsilon)-\omega^{2}} .
$$

This approximation is inherently related to the $m=n$ term (i.e. the contribution for given $\boldsymbol{k}$ of the dispersion branch nearest to the prescribed frequency) in expression (12) for a general forced motion.

Remark 11. Since $\tilde{\lambda}_{n}^{a}(\epsilon)-\omega^{2}=O\left(\epsilon^{2}\right)$ while $\tilde{\lambda}_{m}^{a}(\epsilon)-\omega^{2}=O(1)$ for any $m \neq n$ under the chosen scaling (23), it is clear from (12) that the $O\left(\epsilon^{-2}\right)$ and $O\left(\epsilon^{-1}\right)$ contributions to $\tilde{u}$ are entirely due to the nearest $n$-th branch.

Lemma 5. (a) The effective tensorial coefficient $\boldsymbol{\mu}^{(2)}$ in (56) admits the following expression, involving $\boldsymbol{\chi}^{(1)}$ and $\boldsymbol{\chi}^{(2)}$ but not $\boldsymbol{\chi}^{(3)}$ :

$$
\begin{aligned}
\boldsymbol{\mu}^{(2)}=\left\{\left\langle G \boldsymbol{I} \otimes \boldsymbol{\chi}^{(2)}\right\rangle_{\boldsymbol{a}}^{\varphi}+\frac{1}{\rho^{(0)}} \boldsymbol{\mu}^{(0)} \otimes\left(\rho \boldsymbol{\chi}^{(1)} \otimes \boldsymbol{\chi}^{(1)}, 1\right)_{\overline{Y_{a}}}-\left(G \boldsymbol{I} \otimes \boldsymbol{\chi}^{(1)} \otimes \boldsymbol{\chi}^{(1)}, 1\right)_{\overline{Y_{\boldsymbol{a}}}}\right. \\
\left.+\left(G \nabla \boldsymbol{\chi}^{(1)} \otimes \boldsymbol{\chi}^{(2)}, 1\right)_{\overline{Y_{\boldsymbol{a}}}}-\left(G \boldsymbol{\chi}^{(1)} \otimes \nabla \boldsymbol{\chi}^{(2)}, 1\right)_{\overline{Y_{a}}}\right\}
\end{aligned}
$$

(b) The effective source term $F(\hat{\boldsymbol{k}}, \hat{\omega}, \epsilon)$ has the following alternative expression, involving $\boldsymbol{\chi}^{(1)}, \boldsymbol{\chi}^{(2)}$ and $\eta^{(0)}$ but not $\boldsymbol{\eta}^{(1)}$ :

$$
\begin{aligned}
F(\hat{\boldsymbol{k}}, \hat{\omega}, \epsilon)=\left\langle\tilde{f} e^{i \boldsymbol{k}^{\boldsymbol{a}} \cdot \boldsymbol{x}}\right\rangle_{\boldsymbol{a}}^{\varphi}- & \epsilon\left(\boldsymbol{\chi}^{(1)} \tilde{f} e^{i \boldsymbol{k}^{a} \cdot \boldsymbol{x}}, 1\right)_{\overline{Y_{\boldsymbol{a}}}} \cdot i \hat{\boldsymbol{k}}+\frac{\epsilon^{2}}{\rho^{(0)}}\left(\boldsymbol{\mu}^{(0)}:(i \hat{\boldsymbol{k}})^{2}+\sigma \rho^{(0)} \hat{\omega}^{2}\right)\left\langle\rho \eta^{(0)}\right\rangle_{\boldsymbol{a}}^{\varphi} \\
& +\epsilon^{2}(i \hat{\boldsymbol{k}})^{2}:\left(\boldsymbol{\chi}^{(2)} \tilde{f} e^{i \boldsymbol{k}^{\boldsymbol{a}} \cdot \boldsymbol{x}}, 1\right)_{\overline{Y_{\boldsymbol{a}}}}+\frac{\epsilon^{2}}{\rho^{(0)}}(i \hat{\boldsymbol{k}})^{2}:\left(\left(\rho \boldsymbol{\chi}^{(1)} \otimes \boldsymbol{\chi}^{(1)}, 1\right)_{\overline{Y_{\boldsymbol{a}}}}-\boldsymbol{\rho}^{(2)}\right)\left\langle\tilde{f} e^{i \boldsymbol{k}^{a} \cdot \boldsymbol{x}}\right\rangle_{\boldsymbol{a}}^{\varphi}
\end{aligned}
$$

See Appendix, Section 7 for proof.

For completeness, we note that claim (a) in Lemma 5 is the counterpart for $\boldsymbol{\mu}^{(2)}$ of Lemma 4 , wherein $\boldsymbol{\mu}^{(1)}$ is evaluated without using $\chi^{(2)}$.

Remark 12. The forcing term in (43) is the orthogonal projection of the original forcing $\tilde{f} e^{i \boldsymbol{k}^{a} \cdot \boldsymbol{x}}$ onto $\operatorname{Span}\left(\tilde{\varphi}_{m}^{a}\right), m \neq n$. Moreover, by Lemma $5, F$ is completely determined by $\boldsymbol{\chi}^{(1)}, \boldsymbol{\chi}^{(2)}, \eta^{(0)}$. The cell function $\eta^{(0)}$ introduced in (43), (44) therefore provides the (leading) $O(1)$ contribution to $\tilde{u}$ due to all non-nearest branches. 


\subsection{Effective field equation: Green's function near the edge of a band gap}

To illustrate the utility of the foregoing developments, we consider the Green's function $u$ solving (1) with $f=$ $\delta\left(\boldsymbol{x}-\boldsymbol{x}^{\prime}\right)$ and $\omega$ such that $\omega-\omega_{n}(\boldsymbol{k})=O\left(\epsilon^{2}\right)$ for some $n=\hat{n}$ and $\boldsymbol{k}=\boldsymbol{k}^{\boldsymbol{a}}$, whereas there exists $C>0$ such that we have $\left|\omega-\omega_{n}(\boldsymbol{k})\right| \geq C$ for all other $n$ and all $\boldsymbol{k}$ away from $\boldsymbol{k}^{\boldsymbol{a}}$. In this setting, one finds that the spectrum of $u$ is localized in a neighborhood of $\left(\boldsymbol{k}^{\boldsymbol{a}}, \omega_{\hat{n}}^{\boldsymbol{a}}\right)$. As examined in $[12,10,16]$, this allows us to take advantage the Bloch expansion theorem [4] toward obtaining an effective model of (1).

As will be seen shortly, the featured assumption on $f$ implies that $\tilde{f}$ is also of Dirac delta type, and consequently does not belong to $L^{2}(Y)$ as assumed earlier. In this section, we consequently consider such "Dirac" $\tilde{f}$ as an idealization (i.e. the distributional limit) of an $L^{2}(Y)$ source term with vanishing spatial support, and we further assume (without proof) that the induced effective motion behaves properly in the limit. For clarity of discussion, we also assume that the material properties are smooth in a neighborhood of the source point so that the pointwise eigenfunction values there make sense.

On denoting by $\gamma_{\boldsymbol{a}}$ the lattice vectors of a structure with period $Y_{\boldsymbol{a}}$ and assuming that both $f$ and $u$ in (1) are square integrable over $\mathbb{R}^{d}$, their Bloch wave companions

$$
\tilde{g}(\boldsymbol{x} ; \boldsymbol{k})=\sum_{\boldsymbol{\gamma}_{\boldsymbol{a}}} g\left(\boldsymbol{x}+\boldsymbol{\gamma}_{\boldsymbol{a}}\right) e^{-i \boldsymbol{k} \cdot\left(\boldsymbol{x}+\boldsymbol{\gamma}_{\boldsymbol{a}}\right)}, \quad g=u, f
$$

can be shown to satisfy (6) with $Y$ superseded by $Y_{\boldsymbol{a}}$. In this setting, the Bloch expansion theorem gives

$$
u(\boldsymbol{x})=\left|\mathcal{B}_{\boldsymbol{a}}\right|^{-1} \int_{\boldsymbol{k}_{s}+\mathcal{B}_{a}} \tilde{u}(\boldsymbol{x} ; \boldsymbol{k}) e^{i \boldsymbol{k} \cdot \boldsymbol{x}} \mathrm{d} \boldsymbol{k}, \quad \boldsymbol{x} \in \mathbb{R}^{d}
$$

and similarly for $f$, where $\mathcal{B}_{\boldsymbol{a}}$ is the first Brillouin zone corresponding to $Y_{\boldsymbol{a}}$ and $\boldsymbol{k}_{s} \in \mathbb{R}^{d}$ is an arbitrary shift vector. Following [16], we conveniently let $\boldsymbol{k}_{s}=\boldsymbol{k}^{\boldsymbol{a}}$ and we denote by $\boldsymbol{\gamma}$ the lattice vectors of a structure with period $Y$. By letting $\omega^{2}=\tilde{\lambda}_{\hat{n}}^{a}+\epsilon^{2} \sigma \hat{\omega}^{2}$ for some fixed $\hat{\omega}$, the principal contribution to (58) derives from a neighborhood of $\boldsymbol{k}^{\boldsymbol{a}}$ described by the scaling $\boldsymbol{k}=\boldsymbol{k}^{\boldsymbol{a}}+\epsilon \hat{\boldsymbol{k}}$. From (57) we also find that $\tilde{f}(\boldsymbol{x} ; \boldsymbol{k})=$ $\delta\left(\boldsymbol{x}-\boldsymbol{x}^{\prime}\right) e^{-i\left(\boldsymbol{k}^{a}+\epsilon \hat{\boldsymbol{k}}\right) \cdot \boldsymbol{x}}$ there, whereby Theorem 1 gives

$$
\begin{aligned}
-\left(\boldsymbol{\mu}^{(0)}:(i \epsilon \hat{\boldsymbol{k}})^{2}+\rho^{(0)} \epsilon^{2} \sigma \hat{\omega}^{2}\right)\langle\tilde{u}\rangle_{\boldsymbol{a}} & =\left\langle\delta\left(\boldsymbol{x}-\boldsymbol{x}^{\prime}\right) e^{-i \epsilon \hat{\boldsymbol{k}} \cdot \boldsymbol{x}}\right\rangle_{\boldsymbol{a}}^{\varphi}-\left(\boldsymbol{\chi}^{(1)}(\boldsymbol{x}) \delta\left(\boldsymbol{x}-\boldsymbol{x}^{\prime}\right) e^{-i \epsilon \hat{\boldsymbol{k}} \cdot \boldsymbol{x}}, 1\right)_{\overline{Y_{a}}} \cdot i \epsilon \hat{\boldsymbol{k}} \\
& =\left|Y_{\boldsymbol{a}}\right|^{-1} e^{-i \epsilon \hat{\boldsymbol{k}} \cdot \boldsymbol{x}^{\prime}}\left(\tilde{\varphi}_{\hat{n}}^{\boldsymbol{a}}\left(\boldsymbol{x}^{\prime}\right)-\boldsymbol{\chi}^{(1)}\left(\boldsymbol{x}^{\prime}\right) \cdot i \epsilon \hat{\boldsymbol{k}}\right),
\end{aligned}
$$

upon discarding the second-order correction. For clarity, we denote the solution of (59) by $\langle\tilde{u}\rangle_{\boldsymbol{a}}[\epsilon \hat{\boldsymbol{k}}]$.

By letting $\tilde{\phi}_{\hat{n}}^{a}$ satisfy (14) in every $\gamma+Y$, (58) is next evaluated on the basis of (59) by: (i) applying the local approximation

$$
\tilde{u}\left(\boldsymbol{x} ; \boldsymbol{k}^{\boldsymbol{a}}+\epsilon \hat{\boldsymbol{k}}\right) \simeq\langle\tilde{u}\rangle_{\boldsymbol{a}}[\epsilon \hat{\boldsymbol{k}}] \tilde{\phi}_{\hat{n}}^{\boldsymbol{a}}(\boldsymbol{x}), \quad \boldsymbol{x} \in \mathbb{R}^{d}
$$

uniformly for all $\epsilon \hat{\boldsymbol{k}} \in \mathcal{B}_{\boldsymbol{a}}$, and (ii) extending the domain of integration in (58) to $\mathbb{R}^{d}$, see also [12]. Specifically, we write

$$
u(\boldsymbol{x}) \simeq\left|\mathcal{B}_{\boldsymbol{a}}\right|^{-1} \tilde{\phi}_{\hat{n}}^{\boldsymbol{a}}(\boldsymbol{x}) e^{i \boldsymbol{k}^{a} \cdot \boldsymbol{x}} \int_{\mathcal{B}_{\boldsymbol{a}}}\langle\tilde{u}\rangle_{\boldsymbol{a}}[\epsilon \hat{\boldsymbol{k}}] e^{i \epsilon \hat{\boldsymbol{k}} \cdot \boldsymbol{x}} \mathrm{d}(\epsilon \hat{\boldsymbol{k}}):=\tilde{\varphi}_{\hat{n}}^{\boldsymbol{a}}(\boldsymbol{x}) U_{\boldsymbol{a}}(\boldsymbol{x}),
$$

where $\tilde{\varphi}_{\hat{n}}^{\boldsymbol{a}}=\tilde{\phi}_{\hat{n}}^{\boldsymbol{a}} e^{i \boldsymbol{k}^{\boldsymbol{a}} \cdot \boldsymbol{x}}$ solves (18) in every $\boldsymbol{\gamma}_{\boldsymbol{a}}+Y_{\boldsymbol{a}}$, and

$$
U_{\boldsymbol{a}}(\boldsymbol{x})=\left|\mathcal{B}_{\boldsymbol{a}}\right|^{-1} \int_{\mathcal{B}_{\boldsymbol{a}}}\langle\tilde{u}\rangle_{\boldsymbol{a}}[\epsilon \hat{\boldsymbol{k}}] e^{i \epsilon \hat{\boldsymbol{k}} \cdot \boldsymbol{x}} \mathrm{d}(\epsilon \hat{\boldsymbol{k}}) \simeq\left|\mathcal{B}_{\boldsymbol{a}}\right|^{-1} \int_{\mathbb{R}^{d}}\langle\tilde{u}\rangle_{\boldsymbol{a}}[\epsilon \hat{\boldsymbol{k}}] e^{i \epsilon \hat{\boldsymbol{k}} \cdot \boldsymbol{x}} \mathrm{d}(\epsilon \hat{\boldsymbol{k}}) .
$$

As argued in [16], the above approximations are supported by the facts that: (i) $\langle\tilde{u}\rangle_{\boldsymbol{a}}[\epsilon \hat{\boldsymbol{k}}]=O\left(\left(\epsilon^{2}+\|\epsilon \hat{\boldsymbol{k}}\|^{2}\right)^{-1}\right)$ for $\epsilon \hat{\boldsymbol{k}} \in \mathbb{R}^{d}$, and (ii) relative error due to approximation (60) diminishes fast with $\left\|\boldsymbol{x}-\boldsymbol{x}^{\prime}\right\|$ since the featured phase function has no stationary points. On recalling that $\left|\mathcal{B}^{\boldsymbol{a}}\right|=(2 \pi)^{d} /\left|Y_{\boldsymbol{a}}\right|$, , (59) and (60) demonstrate that $U_{\boldsymbol{a}}$ satisfies the effective field equation

$$
-\left(\boldsymbol{\mu}^{(0)}: \nabla_{\boldsymbol{x}}^{2}+\rho^{(0)}\left(\omega^{2}-\tilde{\lambda}_{\hat{n}}^{\boldsymbol{a}}\right)\right) U_{\boldsymbol{a}}=\left[\tilde{\varphi}_{\hat{n}}^{\boldsymbol{a}}\left(\boldsymbol{x}^{\prime}\right)-\chi^{(1)}\left(\boldsymbol{x}^{\prime}\right) \cdot \nabla_{\boldsymbol{x}}\right] \delta\left(\boldsymbol{x}-\boldsymbol{x}^{\prime}\right)
$$

that, for $d=2, \omega^{2}>\tilde{\lambda}_{\tilde{n}}^{a}$, and $\boldsymbol{\mu}^{(0)}=\mu^{(0)} \boldsymbol{I}$ with $\mu^{(0)}<0$, can be solved explicitly [16] in terms of modified Bessel functions of the second kind. 


\section{Repeated eigenvalues}

In situations when the multiplicity, $Q$, of eigenvalue $\tilde{\lambda}_{n}^{\boldsymbol{a}}=\tilde{\lambda}_{n}\left(\boldsymbol{k}^{\boldsymbol{a}}\right)$ is larger than one, the foregoing analysis must be generalized to account for: (i) multiple free-wave solutions near the apex point $\left(\boldsymbol{k}^{\boldsymbol{a}}, \omega_{n}^{\boldsymbol{a}}=\left(\tilde{\lambda}_{n}^{\boldsymbol{a}}\right)^{1 / 2}\right)$, and (ii) the commensurate distribution of energy (among competing eigenmodes) supplied by the source term $\tilde{f}$. To encompass both linear and parabolic dispersive behaviors near the points of juncture [16], we consider the wavenumber-frequency perturbation

$$
\boldsymbol{k}=\boldsymbol{k}^{\boldsymbol{a}}+\epsilon \hat{\boldsymbol{k}}, \quad \omega^{2}=\tilde{\lambda}_{n}^{\boldsymbol{a}}+\epsilon^{2} \sigma \hat{\omega}^{2}+\epsilon \sigma \breve{\omega}^{2}, \quad \epsilon=o(1), \quad \sigma= \pm 1,
$$

which differs from (23) by the introduction of (alternative) linear scaling parameter $\sigma \breve{\omega}^{2}$.

Remark 13. Here, $\hat{\omega}$ and $\breve{\omega}$ are designed to be used in the "either or" sense, depending on the driving frequency (when $\tilde{f} \neq 0$ ) or the local geometry of the germane dispersion surface (when $\tilde{f}=0$ ). For instance, it will be seen shortly that for dispersion surfaces with locally parabolic (resp. conical) sections, the frequency in those $\boldsymbol{k}$-directions scales as $\omega(\boldsymbol{k})-\tilde{\lambda}_{n}^{a}=\epsilon^{2} \hat{\omega}^{2}$ (resp. $\left.\omega(\boldsymbol{k})-\tilde{\lambda}_{n}^{a}=\epsilon \breve{\omega}^{2}\right)$. The idea is to enter both into the governing equation (24), and then to identify the appropriate frequency scaling (in terms of either $\hat{\omega}=O(1)$ or $\breve{\omega}=O(1)$ ) depending on the excitation and the local eigenfunction structure. For example, such framework allows us to focus on situations where the driving frequency-wavenumber pair is $o(\epsilon)$ away from a given dispersion branch, irrespective of its local (say parabolic of conical) geometry.

By way of (62), (24)-(25) become

$$
\begin{gathered}
-\left(\tilde{\lambda}_{n}^{\boldsymbol{a}}+\epsilon \sigma \breve{\omega}^{2}+\epsilon^{2} \sigma \hat{\omega}^{2}\right) \rho \tilde{u}-\left(\nabla+i \boldsymbol{k}^{\boldsymbol{a}}+\epsilon i \hat{\boldsymbol{k}}\right) \cdot\left(G\left(\nabla+i \boldsymbol{k}^{\boldsymbol{a}}+\epsilon i \hat{\boldsymbol{k}}\right) \tilde{u}\right)=\tilde{f} \quad \text { in } Y, \\
\llbracket \tilde{u} \rrbracket+\kappa^{-1} t_{\boldsymbol{\nu}}^{\boldsymbol{k}^{\boldsymbol{a}}+\epsilon \hat{\boldsymbol{k}}}[\tilde{u}, \tilde{u}]=0 \quad \text { on } \Gamma,\left.\quad 1 t_{\boldsymbol{n}}^{\boldsymbol{k}^{\boldsymbol{a}}+\epsilon \hat{\boldsymbol{k}}}[\tilde{u}, \tilde{u}]\right|_{j} ^{+}=0 \quad \text { on } \partial Y,
\end{gathered}
$$

and we pursue their expansion via the factorized ansatz (26) whose entries are subject to the coupled interfacial and boundary conditions (27).

\subsection{Eigenfunction basis}

Let $\tilde{\phi}_{n q}^{a} \in H_{p}^{1}(Y), q=\overline{1, Q}$ be linearly independent eigenfunctions that are orthogonal in $L_{\rho}^{2}(Y)$ and of unit $L^{2}(Y)$ norm, see (11), and that satisfy (14). Following the analysis in Section 3.3, we let

$$
\tilde{\phi}_{n q}^{a}(\boldsymbol{x})=\tilde{\varphi}_{n q}^{a}(\boldsymbol{x}) e^{-i \boldsymbol{k}^{\boldsymbol{a}} \cdot \boldsymbol{x}}, \quad q=\overline{1, Q}
$$

and we make use of extension (17) so that $\tilde{\varphi}_{n q}^{a} \in H_{p}^{1}\left(Y_{\boldsymbol{a}}\right)$ satisfy

$$
\left(\rho \tilde{\varphi}_{n q}^{\boldsymbol{a}}, \tilde{\varphi}_{n p}^{\boldsymbol{a}}\right)_{Y_{\boldsymbol{a}}}=0 \quad \text { for } q \neq p, \quad\left(\tilde{\varphi}_{n q}^{\boldsymbol{a}}, \tilde{\varphi}_{n q}^{\boldsymbol{a}}\right)_{Y_{\boldsymbol{a}}}=\left|Y_{\boldsymbol{a}}\right|
$$

and solve

$$
\begin{gathered}
-\nabla \cdot\left(G \nabla \tilde{\varphi}_{n q}^{\boldsymbol{a}}\right)=\tilde{\lambda}_{n}^{\boldsymbol{a}} \rho \tilde{\varphi}_{n q}^{\boldsymbol{a}} \text { in } Y_{\boldsymbol{a}}, \\
\llbracket \tilde{\varphi}_{n q}^{\boldsymbol{a}} \rrbracket+\kappa^{-1} t_{\boldsymbol{\nu}}\left[\tilde{\varphi}_{n q}^{\boldsymbol{a}}\right]=0 \text { on } \Gamma_{\boldsymbol{a}},\left.\quad 1 t_{\boldsymbol{n}}\left[\tilde{\varphi}_{n q}^{\boldsymbol{a}}\right]\right|_{\boldsymbol{a}, j} ^{+}=0 \text { on } \partial Y_{\boldsymbol{a}},
\end{gathered}
$$

subject in addition to the internal constraint $\left.\tilde{\varphi}_{n q}^{a}\right|_{x_{j}=0}=-\left.\tilde{\varphi}_{n q}^{a}\right|_{x_{j}=\ell_{j}}$ discussed earlier. By the argument of Lemma 1, we find that $\tilde{\varphi}_{n q}^{a}$ can be taken as real-valued. This claim, however, does not hold for a generic solution to (64) which can be written as a linear combination of $\tilde{\varphi}_{n q}^{a}$ with complex-valued coefficients.

With the foregoing definitions in place, we introduce the ensembles of "averaging" operators

$$
\langle\tilde{u}\rangle_{\boldsymbol{a}}^{q}=\left(\tilde{u}, \tilde{\phi}_{n q}^{\boldsymbol{a}}\right)_{\bar{Y}_{\boldsymbol{a}}}, \quad\langle\tilde{u}\rangle_{\boldsymbol{a}}^{q \varphi}=\left(\tilde{u}, \tilde{\varphi}_{n q}^{\boldsymbol{a}}\right)_{\overline{Y_{a}}}, \quad q=\overline{1, Q},
$$

and we generalize the second of (22) as

$$
H_{p 0}^{1 a}\left(Y_{\boldsymbol{a}}\right)=\left\{g \in H_{p}^{1}\left(Y_{\boldsymbol{a}}\right):\langle g\rangle_{\boldsymbol{a}}^{q \varphi}=0, q=\overline{1, Q}\right\} .
$$

Remark 14. In the sequel, we do not employ implicit summation over repeated indexes $p, q, r$ and $s$. We shall also assume $1 \leqslant p, q, r, s \leqslant Q$ unless stated otherwise. 


\subsection{Elements of the leading-order approximation}

By virtue of (63) and (26)-(27), we find that $\tilde{u}_{0} \in H_{p}^{1}\left(Y_{\boldsymbol{a}}\right)$ solves the $O\left(\epsilon^{-2}\right)$ system (29). A comparison with (64) shows that

$$
\tilde{u}_{0}(\boldsymbol{x})=\sum_{q} u_{0 q} \tilde{\varphi}_{n q}^{\boldsymbol{a}}(\boldsymbol{x})
$$

where $u_{0 q} \in \mathbb{C}(q=\overline{1, Q})$ are constants to be determined.

At the next order of approximation, the $O\left(\epsilon^{-1}\right)$ statement of (63) is given by

$$
\begin{gathered}
-\tilde{\lambda}_{n}^{\boldsymbol{a}} \rho \tilde{u}_{1}-\sigma \breve{\omega}^{2} \rho \tilde{u}_{0}-\nabla \cdot\left(G\left(\nabla \tilde{u}_{1}+i \hat{\boldsymbol{k}} \tilde{u}_{0}\right)\right)-i \hat{\boldsymbol{k}} \cdot\left(G \nabla \tilde{u}_{0}\right)=0 \quad \text { in } Y_{\boldsymbol{a}}, \\
\llbracket \tilde{u}_{1} \rrbracket+\kappa^{-1} t_{\boldsymbol{\nu}}^{\hat{\boldsymbol{k}}}\left[\tilde{u}_{1}, \tilde{u}_{0}\right]=0 \quad \text { on } \Gamma_{\boldsymbol{a}},\left.\quad 1 t_{\boldsymbol{n}}^{\hat{\boldsymbol{k}}}\left[\tilde{u}_{1}, \tilde{u}_{0}\right]\right|_{\boldsymbol{a}, j} ^{+}=0 \quad \text { on } \partial Y_{\boldsymbol{a}} .
\end{gathered}
$$

On evaluating the weighed integral $\langle(68)\rangle_{\boldsymbol{a}}^{p \varphi}$, we find by following the proof of Lemma 2 that

$$
\sum_{q} \boldsymbol{\theta}_{p q}^{(0)} \cdot(i \hat{\boldsymbol{k}}) u_{0 q}+\sigma \breve{\omega}^{2} \rho_{p}^{(0)} u_{0 p}=0, \quad p=\overline{1, Q}
$$

where

$$
\rho_{p}^{(0)}=\left\langle\rho \tilde{\varphi}_{n p}^{a}\right\rangle_{\boldsymbol{a}}^{p \varphi}, \quad \boldsymbol{\theta}_{p q}^{(0)}=\left\langle G \nabla \tilde{\varphi}_{n q}^{\boldsymbol{a}}\right\rangle_{\boldsymbol{a}}^{p \varphi}-\left\langle G \nabla \tilde{\varphi}_{n p}^{\boldsymbol{a}}\right\rangle_{\boldsymbol{a}}^{q \varphi}, \quad p, q=\overline{1, Q}
$$

Here it is useful to note as in [16] that the leading-order mean energy density of a Bloch wave $\tilde{u}$, averaged in space and time [34], is given by

$$
\bar{E}_{0}=\frac{1}{2} \omega^{2}\left(\rho \tilde{u}_{0}, \tilde{u}_{0}\right)=\frac{1}{2} \omega^{2} \sum_{q} \rho_{q}^{(0)} u_{0 q}^{2}
$$

whereby the decomposition (67) is energy-preserving.

To facilitate the analysis, one can recast (69) as the generalized eigenvalue problem

$$
\sum_{q} A_{p q} u_{0 q}-\tau \sum_{q} D_{p q} u_{0 q}=0, \quad p=\overline{1, Q}
$$

where

$$
A_{p q}=\boldsymbol{\theta}_{p q}^{(0)} \cdot i \hat{\boldsymbol{k}}, \quad D_{p q}=\delta_{p q} \rho_{q}^{(0)} \quad \tau=-\sigma \breve{\omega}^{2} .
$$

Due to the facts that $\boldsymbol{\theta}_{p q}^{(0)} \in \mathbb{R}^{d}$ and $\rho_{q}^{(0)}>0$, we see that $\left[A_{p q}\right]$ is Hermitian skew-symmetric, and that $\left[D_{p q}\right]$ is positive definite.

Remark 15. The following statements hold: (i) every $\tau$ solving (71) is either real-valued or zero; (ii) if $\tau$ is an eigenvalue of (71), so is $-\tau$; (iii) the eigenvectors corresponding to $\tau$ and $-\tau$ are complex conjugates of each other, and (iv) the maximum rank of $A_{p q}$ is $Q$ (resp. Q-1) for $Q$ even (resp. odd). See [16] for proof.

Assumption 1. Let $\tau_{r}(r=\overline{1, Q})$ denote the eigenvalues of (71). To provide a focus for the analysis, we assume that the driving frequency $\omega$ (characterizing the source term) is such that $\left|\tau-\tau_{r}\right|>C$ for all $r$ and some $C>0$, where $\tau=-\sigma \breve{\omega}^{2}=-\left(\omega^{2}-\tilde{\lambda}_{n}^{a}\right) / \epsilon$. As will be seen shortly, the condition $\tau \neq \tau_{r}(r=\overline{1, Q})$ is a sufficient condition for the well-posedness of the ensuing effective model. Later on, we will also investigate the situations where $\tau-\tau_{r}=O(\epsilon)$ for some $r$.

Remark 16. In the sequel we seek, whenever possible, a non-trivial solution to (71). In principle, the latter system of equations should be satisfied identically because $u_{0 q}$, when non-trivial, are also required to satisfy higher-order equations with a source term. As it turns out, this guiding principle is consistent with the goals stated in Remark 13 and helps us distil the frequency scaling law in (62) featuring two alternative perturbation parameters, $\breve{\omega}$ and $\hat{\omega}$. This brings a spotlight on $A_{p q}$ as the lynchpin of our asymptotic analysis, and we proceed with the underpinning expansion for several canonical situations. 


\subsection{Effective model for full-rank $A_{p q}$}

In this case, which by Remark 15 requires $Q$ to be even, the generalized eigenvalue problem (71) has no zero eigenvalues. Consequently, setting $\breve{\omega}=0$ in (62) does not help turn (71) into an identity. To meet the latter criterion, we must let $u_{0 q}=0(q=\overline{1, Q})$, by which the leading-order statement of (63) is $O\left(\epsilon^{-1}\right)$; it reads

$$
\begin{gathered}
-\tilde{\lambda}_{n}^{\boldsymbol{a}} \rho \tilde{u}_{1}-\nabla \cdot\left(G\left(\nabla \tilde{u}_{1}\right)\right)=0 \quad \text { in } Y_{\boldsymbol{a}}, \\
\llbracket \tilde{u}_{1} \rrbracket+\kappa^{-1} t_{\boldsymbol{\nu}}\left[\tilde{u}_{1}\right]=0 \quad \text { on } \Gamma_{\boldsymbol{a}},\left.\quad 1 t_{\boldsymbol{n}}\left[\tilde{u}_{1}\right]\right|_{\boldsymbol{a}, j} ^{+}=0 \quad \text { on } \partial Y_{\boldsymbol{a}} .
\end{gathered}
$$

A general solution to this problem is given by

$$
\tilde{u}_{1}(\boldsymbol{x})=\sum_{q} u_{1 q} \tilde{\varphi}_{n q}^{\boldsymbol{a}}(\boldsymbol{x}),
$$

where $u_{1 q} \in \mathbb{C}(q=\overline{1, Q})$ are constants.

To compute $u_{1 q}$, we identify the $O(1)$ statement of $(63)$ as

$$
\begin{gathered}
-\tilde{\lambda}_{n}^{\boldsymbol{a}} \rho \tilde{u}_{2}-\nabla \cdot\left(G\left(\nabla \tilde{u}_{2}+i \hat{\boldsymbol{k}} \tilde{u}_{1}\right)\right)-i \hat{\boldsymbol{k}} \cdot\left(G \nabla \tilde{u}_{1}\right)-\sigma \breve{\omega}^{2} \rho \tilde{u}_{1}=\tilde{f} e^{i \boldsymbol{k}^{\boldsymbol{a}} \cdot \boldsymbol{x}} \quad \text { in } Y_{\boldsymbol{a}}, \\
\llbracket \tilde{u}_{2} \rrbracket+\kappa^{-1} t_{\boldsymbol{\nu}}^{\hat{\boldsymbol{k}}}\left[\tilde{u}_{2}, \tilde{u}_{1}\right]=0 \quad \text { on } \Gamma_{\boldsymbol{a}},\left.\quad 1 t_{\boldsymbol{n}}^{\hat{\boldsymbol{k}}}\left[\tilde{u}_{2}, \tilde{u}_{1}\right]\right|_{\boldsymbol{a}, j} ^{+}=0 \quad \text { on } \partial Y_{\boldsymbol{a}} .
\end{gathered}
$$

On evaluating the weighed integral $\langle(75)\rangle_{\boldsymbol{a}}^{p \varphi}(p=\overline{1, Q})$ as in the treatment of (35), we find

$$
\left(i \hat{\boldsymbol{k}} G \tilde{u}_{1}, \nabla \tilde{\varphi}_{n p}^{\boldsymbol{a}}\right)_{\overline{Y_{\boldsymbol{a}}}}-\left(i \hat{\boldsymbol{k}} \cdot\left(G \nabla \tilde{u}_{1}\right), \tilde{\varphi}_{n p}^{\boldsymbol{a}}\right)_{\overline{Y_{\boldsymbol{a}}}}-\sigma \breve{\omega}^{2}\left(\rho \tilde{u}_{1}, \tilde{\varphi}_{n p}^{\boldsymbol{a}}\right)_{\overline{Y_{\boldsymbol{a}}}}=\left(\tilde{f} e^{i \boldsymbol{k}^{\boldsymbol{a}} \cdot \boldsymbol{x}}, \tilde{\varphi}_{n p}^{\boldsymbol{a}}\right)_{\overline{Y_{\boldsymbol{a}}}} \cdot
$$

A substitution of (74) into (76) yields a system of $Q$ equations for $u_{1 p}$ as

$$
-\sum_{q} A_{p q} u_{1 q}-\sigma \breve{\omega}^{2} \sum_{q} D_{p q} u_{1 q}=\left\langle\tilde{f} e^{i \boldsymbol{k}^{\boldsymbol{a}} \cdot \boldsymbol{x}}\right\rangle_{\boldsymbol{a}}^{p \varphi}, \quad p=\overline{1, Q}
$$

where $A_{p q}$ and $D_{p q}$ are given by (72). We note that this problem is well-posed thanks to Assumption 1.

Remark 17. By Remark 15, the generalized eigenvalue problem (71) underpinning (77) has $Q$ real eigenvalues, hereon denoted by $\tau_{r}=-\sigma_{r} \breve{\omega}_{r}^{2}(r=\overline{1, Q})$, that appear in " \pm " pairs. Regarding the free waves, there are $Q / 2$ pairs of dispersion branches emanating from the apex point $\left(\boldsymbol{k}^{\boldsymbol{a}}, \omega_{n}^{\boldsymbol{a}}\right)$; they are given by mappings $\left(\boldsymbol{k}^{\boldsymbol{a}}+\epsilon \hat{\boldsymbol{k}}, \omega_{n}^{\boldsymbol{a}}+\epsilon \sigma_{r} \breve{\omega}_{r}^{2}(\hat{\boldsymbol{k}}) /\left(2 \omega_{n}^{\boldsymbol{a}}\right)\right)$. Each such branch is characterized by an $O(1)$ (positive or negative) group velocity, and is stenciled by the respective eigenfunction $\sum_{q} e_{q}^{r} \tilde{\varphi}_{n q}^{a}$, where $e_{q}^{r} \in \mathbb{C}(q=\overline{1, Q})$ are components of the unit eigenvector of $A_{p q}$ corresponding to $\tau=-\sigma_{r} \breve{\omega}_{r}^{2}$. On the other hand, when solving for $u_{1 p}$, we implicitly assume $\sigma \breve{\omega}^{2} \neq \sigma_{r} \breve{\omega}_{r}^{2}(r=\overline{1, Q})$, i.e. exclude scalings (62) which to leading order lie on a linearized dispersion branch.

\subsection{Effective model for (near-) trivial $A_{p q}$}

In certain situations, matrix $A_{p q}$ in (71) may either vanish for all $\hat{\boldsymbol{k}}$, or become exceedingly small for some (but not all) perturbation directions $\hat{\boldsymbol{k}}$. These two situations occur respectively when: $\boldsymbol{\theta}_{p q}^{(0)}=\mathbf{0}(p, q=\overline{1, Q})$ due to inherent symmetries of the unit cell, and $\boldsymbol{\theta}_{p q}^{(0)}(p, q=\overline{1, Q}, p \neq q)$ are parallel so that $A_{p q}$ vanishes as $\hat{\boldsymbol{k}}$ becomes orthogonal to $\boldsymbol{\theta}_{p q}^{(0)}$. To illustrate the situation, we assume $A_{p q}=O(\epsilon)$, and we define the "residual" as RHS - LHS. In such instances the effective $O\left(\epsilon^{-1}\right)$ statement of (63), computed by integrating

$$
\left\langle-\tilde{\lambda}_{n}^{\boldsymbol{a}} \rho \tilde{u}_{1}-\nabla \cdot\left(G\left(\nabla \tilde{u}_{1}+i \hat{\boldsymbol{k}} \tilde{u}_{0}\right)\right)-i \hat{\boldsymbol{k}} \cdot\left(G \nabla \tilde{u}_{0}\right)-\rho \sigma \breve{\omega}^{2} \tilde{u}_{0}\right\rangle_{\boldsymbol{a}}^{p \varphi}=0, \quad p=\overline{1, Q}
$$

reads

$$
-\sigma \breve{\omega}^{2} \sum_{q} D_{p q} u_{0 q}=0, \quad p=\overline{1, Q}
$$

which: (i) requires that $\breve{\omega}=0$ to preserve the leading-order solution, and (ii) discards the $O(\epsilon)$ residual

$$
-\epsilon \sum_{q} \bar{A}_{p q} u_{0 q}, \quad \bar{A}_{p q}=\epsilon^{-1} A_{p q}, \quad p=\overline{1, Q}
$$


that carries over to the next order of effective approximation. In this setting, the problem governing $\tilde{u}_{1}$ is given by (31), which yield

$$
\tilde{u}_{1}(\boldsymbol{x})=\sum_{q} u_{0 q} \boldsymbol{\chi}_{q}^{(1)}(\boldsymbol{x}) \cdot i \hat{\boldsymbol{k}}+\sum_{q} u_{1 q} \tilde{\varphi}_{n q}^{\boldsymbol{a}}(\boldsymbol{x}),
$$

where $u_{1 q} \in \mathbb{C}(q=\overline{1, Q})$ are constants and $\boldsymbol{\chi}_{q}^{(1)} \in\left(H_{p 0}^{1 a}\left(Y_{\boldsymbol{a}}\right)\right)^{d}$ uniquely solve

$$
\begin{aligned}
& \tilde{\lambda}_{n}^{a} \rho \boldsymbol{\chi}_{q}^{(1)}+\nabla \cdot\left(G\left(\nabla \boldsymbol{\chi}_{q}^{(1)}+\boldsymbol{I} \tilde{\varphi}_{n q}^{a}\right)\right)+G \nabla \tilde{\varphi}_{n q}^{a}-\sum_{r} \frac{1}{\rho_{r}^{(0)}} \boldsymbol{\theta}_{r q}^{(0)} \rho \tilde{\varphi}_{n r}^{a}=\mathbf{0} \quad \text { in } \quad Y_{\boldsymbol{a}}, \quad q=\overline{1, Q} \\
& \llbracket \boldsymbol{\chi}_{q}^{(1)} \rrbracket+\kappa^{-1} \boldsymbol{t}_{\boldsymbol{\nu}}^{I}\left[\boldsymbol{\chi}_{q}^{(1)}, \tilde{\varphi}_{n q}^{\boldsymbol{a}}\right]=\mathbf{0} \quad \text { on } \Gamma_{\boldsymbol{a}}, \quad 1 \boldsymbol{t}_{\boldsymbol{n}}^{I}\left[\boldsymbol{\chi}_{q}^{(1)}, \tilde{\varphi}_{n q}^{\boldsymbol{a}}\right] \mathrm{L}_{\boldsymbol{a}, j}^{+}=\mathbf{0} \quad \text { on } \partial Y_{\boldsymbol{a}} .
\end{aligned}
$$

Remark 18. By design, the last term in (82) ensures that the source term driving $\boldsymbol{\chi}_{q}^{(1)}$ is orthogonal to $\tilde{\varphi}_{n r}^{a}$ $(r=\overline{1, Q})$ - rendering the system solvable, while maintaining the aforestated effective description. Indeed, on substituting (81) into (78), we recover precisely the $O(\epsilon)$ effective equation (79) and its residual (80). The situation is consistent in terms of the local equations: a direct substitution of (81) into (31) generates the $O(\epsilon)$ residual

$$
-\epsilon \sum_{q} u_{0 q} \sum_{r} \frac{1}{\rho_{r}^{(0)}} \bar{A}_{r q} \rho(\boldsymbol{x}) \tilde{\varphi}_{n r}^{a}(\boldsymbol{x})
$$

in the field equation, whose multiplication by $\tilde{\varphi}_{n p}^{\boldsymbol{a}}(p=\overline{1, Q})$ and integration over $Y_{\boldsymbol{a}}$ recovers the "effective" residuals in (80).

We next substitute (81) into (35)-(36) and integrate $\langle(35)\rangle_{\boldsymbol{a}}^{p \varphi}$ by parts to obtain the $O(1)$ effective equation, that includes the "carryover" (80), as

$$
-\sum_{q}\left(B_{p q}+\bar{A}_{p q}\right) u_{0 q}-\sigma \hat{\omega}^{2} \sum_{q} D_{p q} u_{0 q}=\left\langle\tilde{f} e^{i \boldsymbol{k}^{\boldsymbol{a}} \cdot \boldsymbol{x}}\right\rangle_{\boldsymbol{a}}^{p \varphi}, \quad p=\overline{1, Q},
$$

where $D_{p q}$ is given by (72), and

$$
B_{p q}=\boldsymbol{\mu}_{p q}^{(0)}:(i \hat{\boldsymbol{k}})^{2}, \quad \boldsymbol{\mu}_{p q}^{(0)}=\left\langle G\left\{\nabla \boldsymbol{\chi}_{q}^{(1)}+\boldsymbol{I} \tilde{\varphi}_{n q}^{\boldsymbol{a}}\right\}\right\rangle_{\boldsymbol{a}}^{p \varphi}-\left(G\left\{\boldsymbol{\chi}_{q}^{(1)} \otimes \nabla \tilde{\varphi}_{n p}^{\boldsymbol{a}}\right\}, 1\right)_{\bar{Y}_{\boldsymbol{a}}} .
$$

Note that (83) discards the residual

$$
-\epsilon \sum_{q} \bar{A}_{p q} u_{1 q}
$$

which inherently enters the $O(\epsilon)$ effective equation.

Lemma 6. Effective matrix $B_{p q}$ is symmetric. See Appendix, Section 7 for proof.

Remark 19. In situations where $A_{p q}=0$ and thus $\bar{A}_{p q}=0$ due to trivial $\boldsymbol{\theta}_{p q}^{(0)}$, we see from (83) that the effective motion components $u_{0 q}$ are governed by a pure "second-order" system whose impedance coefficients are quadratic in $i \hat{\boldsymbol{k}}$. In this case, the sections of the dispersion surfaces $\hat{\omega}(\hat{\boldsymbol{k}})$ in each direction $\hat{\boldsymbol{k}} /|\hat{\boldsymbol{k}}|$ are locally parabolic with a vertex at $\hat{\boldsymbol{k}}=\mathbf{0}$. In contrast, for vanishing but non-zero $A_{p q}$ (generated by parallel $\boldsymbol{\theta}_{p q}^{(0)}$ and near-orthogonal $\hat{\boldsymbol{k}}),(83)$ provides a link with $(77)$ in the following way. First, we recall the scaling law (62) to establish a connection between $\sigma \breve{\omega}^{2}$ in (77) and $\sigma \hat{\omega}^{2}$ in (83) as

$$
\sigma \breve{\omega}^{2}=\frac{\omega^{2}-\tilde{\lambda}_{n}^{a}}{\epsilon}, \quad \sigma \hat{\omega}^{2}=\frac{\omega^{2}-\tilde{\lambda}_{n}^{a}}{\epsilon^{2}}, \quad \Longrightarrow \quad \sigma \hat{\omega}^{2}=\epsilon^{-1} \sigma \breve{\omega}^{2} .
$$

For clarity, we note that (77) (resp. (83)) assumes $\hat{\omega}=0$ (resp. $\breve{\omega}=0$ ), which is the basis for the formal relationship established in (85). Next, we let $\hat{\boldsymbol{k}}$ in (83) depart from the normal to $\boldsymbol{\theta}_{p q}^{(0)}$ so that $A_{p q} \rightarrow O(1)$. By virtue of (85) and definition $\bar{A}_{p q}=\epsilon^{-1} A_{p q},(83)$ degenerates to

$$
-\sum_{q} A_{p q} u_{0 q}-\sigma \breve{\omega}^{2} \sum_{q} D_{p q} u_{0 q}=\epsilon\left\langle\tilde{f} e^{i \boldsymbol{k}^{a} \cdot \boldsymbol{x}}\right\rangle_{\boldsymbol{a}}^{p \varphi}, \quad p=\overline{1, Q}
$$


which carries an $O(\epsilon)$ operator residual

$$
-\epsilon \sum_{q} B_{p q} u_{0 q}
$$

and recovers (77) on letting $u_{0 q}=\epsilon u_{1 q}, q=\overline{1, Q}$. The latter equality in fact synthesizes the transition in the order of approximation between (77) and (83), as driven by either diminishing $u_{0 q}$ (taking $\hat{\boldsymbol{k}} /\|\hat{\boldsymbol{k}}\|$ in (83) s.th. $A_{p q}: O(\epsilon) \rightarrow O(1)$ ) or "growing" $u_{1 q}$ (choosing $\hat{\boldsymbol{k}} /\|\hat{\boldsymbol{k}}\|$ in (77) s.th. $A_{p q}: O(1) \rightarrow O(\epsilon)$ ).

\subsection{Effective model for partial-rank $A_{p q}$}

We finally consider a generic case when the generalized eigenvalue problem (71) has $N_{0}$ zero eigenvalues and $N-N_{0}$ vanishing $O(\epsilon)$ eigenvalues, where $0 \leqslant N_{0} \leqslant N \leqslant Q$. Thanks to Remark 15, one finds that $Q-N$ and $N-N_{0}$ are necessarily even. In the sequel, we arrange the eigenvalues so that $\left|\tau_{r}\right| \geqslant\left|\tau_{q}\right|$ for $r>q$, and $\tau_{r+1}=-\tau_{r}$ for $r-N_{0}$ positive and odd. Since $A_{p q}$ is antisymmetric, one may conveniently introduce the factorization [e.g. 15]

$$
A_{p q}=\sum_{r, s} U_{p r} \Sigma_{r s} U_{s q}^{\mathrm{T}}
$$

where $U_{p r}$ is a real-valued orthogonal matrix; "T" denotes matrix transpose;

$$
\left[\Sigma_{r s}\right]=\operatorname{diag}\{\mathbb{O}_{N_{0}}, \underbrace{\left[\begin{array}{cc}
0 & i \tau_{N_{0}+1} \\
-i \tau_{N_{0}+1} & 0
\end{array}\right], \ldots\left[\begin{array}{cc}
0 & i \tau_{N} \\
-i \tau_{N} & 0
\end{array}\right]}_{O(\epsilon)}, \underbrace{\left.\left[\begin{array}{cc}
0 & i \tau_{N+1} \\
-i \tau_{N+1} & 0
\end{array}\right], \ldots\left[\begin{array}{cc}
0 & i \tau_{Q} \\
-i \tau_{Q} & 0
\end{array}\right]\right\}}_{O(1)}
$$

is a $2 \times 2$ block-diagonal matrix, and $\mathbb{O}_{N_{0}}$ stands for an $N_{0} \times N_{0}$ block of zeros.

Remark 20. To simplify the analysis, we select the eigenfunction basis $\tilde{\varphi}_{n q}^{a}(q=\overline{1, Q})$ such that $U_{p q}=\delta_{p q}$. On recalling the definition of $\rho_{q}^{(0)}$ in (70), this can be accommodated via coordinate transformation

$$
\tilde{\psi}_{n q}^{\boldsymbol{a}}(\boldsymbol{x})=c_{q} \sum_{s}\left(\rho_{s}^{(0)}\right)^{-1 / 2} U_{s q} \tilde{\varphi}_{n s}^{\boldsymbol{a}}(\boldsymbol{x}), \quad q=\overline{1, Q},
$$

which maintains both (i) the orthogonality of the new basis $\tilde{\psi}_{n q}^{a}$ in $L_{\rho}^{2}\left(Y_{\boldsymbol{a}}\right)$, and (ii) the previously adopted normalization $\left(\tilde{\psi}_{n q}^{a}, \tilde{\psi}_{n q}^{a}\right)_{Y_{a}}=\left|Y_{a}\right|$ via the choice of $c_{q} \in \mathbb{R}, q=\overline{1, Q}$. In line with the stated objective, we then relabel $\tilde{\psi}_{n q}^{\boldsymbol{a}}$ as $\tilde{\varphi}_{n q}^{\boldsymbol{a}}$ so that

$$
A_{p q}=\Sigma_{p q}
$$

according to (86).

With the above definitions the $Q \times Q$ system of $O\left(\epsilon^{-1}\right)$ effective equations, given by the leading-order statements of $\langle(68)\rangle_{\boldsymbol{a}}^{p \varphi}(p=\overline{1, Q})$, can be shown to read

$$
-\sum_{q>N} A_{p q} u_{0 q}-\sigma \breve{\omega}^{2} \sum_{q} D_{p q} u_{0 q}=0, \quad p=\overline{1, Q}
$$

and to carry forward the $O(\epsilon)$ residuals

$$
-\epsilon \sum_{q \leqslant N} \bar{A}_{p q} u_{0 q}, \quad \bar{A}_{p q}=\epsilon^{-1} A_{p q}, \quad p=\overline{1, N} .
$$

Note the upper limit $N$ on index $p$ in (88) because $A_{p q}=0$ for $q \leqslant N$ and $p>N$.

Remark 21. When considering the free wave motion $(\tilde{f}=0)$, the last $Q-N$ equations in (87) constitute an eigenvalue problem governing (to the leading order) the $Q-N$ conical dispersion branches near $\boldsymbol{k}^{\boldsymbol{a}}$. When $\tilde{f} \neq 0$, on the other hand, $\omega$ is given in which case its scaled expressions $-\hat{\omega}$ and $\breve{\omega}$ - are related $1-1$ according to (85). In what follows we examine the latter situation, including a side remark on how to evaluate the remaining $N$ "parabolic" dispersion branches. 
In this setting, (87) becomes an identity (to the leading order) if (i) $\sigma \breve{\omega}^{2}=O(\epsilon)$ i.e. $\sigma \hat{\omega}^{2}=O(1)$, and (ii) $u_{0 q}=0$ for all $q>N$, whereby

$$
\tilde{u}_{0}(\boldsymbol{x})=\sum_{q \leqslant N} u_{0 q} \tilde{\varphi}_{n q}^{\boldsymbol{a}}(\boldsymbol{x}) .
$$

From (68) with $\sigma \breve{\omega}^{2}=O(\epsilon)$ and (89), we find that

$$
\tilde{u}_{1}(\boldsymbol{x})=\sum_{q \leqslant N} u_{0 q} \boldsymbol{\chi}_{q}^{(1)}(\boldsymbol{x}) \cdot i \hat{\boldsymbol{k}}+\sum_{q} u_{1 q} \tilde{\varphi}_{n q}^{\boldsymbol{a}}(\boldsymbol{x}),
$$

where $u_{1 q} \in \mathbb{C}$ are constants and $\boldsymbol{\chi}_{q}^{(1)} \in\left(H_{p 0}^{1 a}\left(Y_{\boldsymbol{a}}\right)\right)^{d}$ uniquely solve (82) for $q \leqslant N$, see also Remark 18. For completeness, we note that the case $\sigma \breve{\omega}^{2}=O(1)$ - when the driving frequency is closer (qualitatively speaking) to the cones than "parabolas" - cannot be addressed by the current framework. In principle, such problem could be tackled by including, as needed, the lower-order terms $O\left(\epsilon^{n}\right), n<-2$ in factorized ansatz (26).

On deploying (90) and (82) in (35)-(36) and integrating $\langle(35)\rangle_{\boldsymbol{a}}^{p \varphi}, p=\overline{1, N}$ by parts, we obtain the $N \times N$ system of $O(1)$ effective equations

$$
-\sum_{q \leqslant N}\left(B_{p q}+\bar{A}_{p q}\right) u_{0 q}-\sigma \hat{\omega}^{2} \sum_{q \leqslant N} D_{p q} u_{0 q}=\left\langle\tilde{f} e^{i \boldsymbol{k}^{\boldsymbol{a}} \cdot \boldsymbol{x}}\right\rangle_{\boldsymbol{a}}^{p \varphi}, \quad p=\overline{1, N}
$$

that account for (88), where $D_{p q}$ and $B_{p q}$ are given respectively by (72) and (84). Similar to the preceding order of approximation, (91) features the residual

$$
-\epsilon \sum_{q \leqslant N} \bar{A}_{p q} u_{1 q}, \quad p=\overline{1, N}
$$

which enters the $O(\epsilon)$ effective equation. When $\tilde{f}=0,(91)$ constitute an eigenvalue problem for governing the $N$ "parabolic" dispersion branches.

Inherently, the above leading-order model is incomplete for it features only $N$ projections, $u_{0 q}$, at an apex point that entails $Q$ eigenfunctions. Such partial description is for instance insufficient when the right-hand side of (91) vanishes. To expose the remaining $Q-N$ projections in terms of $u_{1 q}$, we revisit the $O(1)$ statement of (63) assuming $\sigma \breve{\omega}^{2}=O(\epsilon)$, namely

$$
\begin{gathered}
-\tilde{\lambda}_{n}^{\boldsymbol{a}} \rho \tilde{u}_{2}-\nabla \cdot\left(G\left(\nabla \tilde{u}_{2}+i \hat{\boldsymbol{k}} \tilde{u}_{1}\right)\right)-i \hat{\boldsymbol{k}} \cdot\left(G\left(\nabla \tilde{u}_{1}+i \hat{\boldsymbol{k}} \tilde{u}_{0}\right)\right)-\sigma \hat{\omega}^{2} \rho \tilde{u}_{0}=\tilde{f} e^{i \boldsymbol{k}^{\boldsymbol{a}} \cdot \boldsymbol{x}} \quad \text { in } Y_{\boldsymbol{a}}, \\
\llbracket \tilde{u}_{2} \rrbracket+\kappa^{-1} t_{\boldsymbol{\nu}}^{\hat{\boldsymbol{k}}}\left[\tilde{u}_{2}, \tilde{u}_{1}\right]=0 \quad \text { on } \Gamma_{\boldsymbol{a}},\left.\quad 1 t_{\boldsymbol{n}}^{\hat{\boldsymbol{k}}}\left[\tilde{u}_{2}, \tilde{u}_{1}\right]\right|_{\boldsymbol{a}, j} ^{+}=0 \quad \text { on } \partial Y_{\boldsymbol{a}} .
\end{gathered}
$$

Analogous to the treatment of (35)-(36) that resulted in (91), we next integrate $\langle(92)\rangle_{\boldsymbol{a}}^{p \varphi}$ for $p>N$ to obtain the $(Q-N) \times(Q-N)$ system of $O(1)$ effective equations

$$
-\sum_{q>N} A_{p q} u_{1 q}=\left\langle\tilde{f} e^{i \boldsymbol{k}^{\boldsymbol{a}} \cdot \boldsymbol{x}}\right\rangle_{\boldsymbol{a}}^{p \varphi}+\sum_{q \leqslant N} B_{p q} u_{0 q}, \quad p=\overline{N+1, Q},
$$

which is solvable thanks to the premise on $A_{p q}$. Note that the response of $u_{1 q}, q=\overline{N+1, Q}$ to $\tilde{f}$ can be qualified as "quasi-static" for it lacks the inertial term (linear in $\sigma \breve{\omega}^{2}$ ) on the left-hand side of the equation.

Remark 22. When $A_{p q}=\Sigma_{p q}$ according to (86) (featuring $N_{0}$ zero eigenvalues and $N-N_{0} O(\epsilon)$ eigenvalues), the "full" effective model that captures the leading-order contribution of each eigenfunction $\tilde{\varphi}_{n q}^{a}(q=\overline{1, Q})$ is given, for driving frequencies close to "a parabolic" branch, by $u_{0 q}, q=\overline{1, N}$ solving (91) and $u_{1 q}, q=\overline{N+1, Q}$ solving (93). In general, steps (87)-(93) can be adapted to accommodate driving frequencies that are either close to a given conical branch, or well separated from all branches in the cluster. For instance taking $\breve{\omega}^{2}=\tau_{q}+O(\epsilon)$ with $q>N$, see (86), would yield an $O\left(\epsilon^{-2}\right)$ projection for that cone and $O\left(\epsilon^{-1}\right)$ projections otherwise. Similarly, assuming a "well-separated" driving frequency would generate an $O\left(\epsilon^{-1}\right)$ from each branch in the cluster. For completeness, we note that the existence of this class of configurations (termed Dirac-like points) at $\boldsymbol{k}^{\boldsymbol{a}}=\mathbf{0}$ with $N_{0}=0, N=1$ and $Q=3$ is directly tied to the phenomenon of zero-index metamaterials [2].

Remark 23. In situations when $N=Q$, (91) recovers the (near-) trivial $A_{p q}$ model (83). On the other hand, letting $N=0$ degenerates (93) to the full-rank $A_{p q}$ model (77). 
4.6. Degenerate case: point $\left(\boldsymbol{k}^{\boldsymbol{a}}+\epsilon \hat{\boldsymbol{k}}, \tilde{\lambda}_{n}^{\boldsymbol{a}}+\epsilon \sigma \breve{\omega}^{2}\right)$ close to one of the dispersion branches

With reference to (71) and Assumption 1, we finally illustrate the case when $\sigma \breve{\omega}^{2}+\tau_{r}=O(\epsilon)$ for some $r \in\{1,2, \ldots Q\}$. We begin by assuming $\operatorname{rank}\left(A_{p q}\right)=Q$ and conveniently rewriting (71) as

$$
-\sum_{q} A_{p q} u_{0 q}-\sigma \breve{\omega}^{2} \sum_{q} D_{p q} u_{0 q}=0, \quad p=\overline{1, Q}
$$

where the sign of the left-hand side is reversed in order to match that of (68). This system is satisfied up to an $O(\epsilon)$ residual

$$
\left(\sigma \breve{\omega}^{2}+\tau_{r}\right) \sum_{q} D_{p q} u_{0 q}, \quad p=\overline{1, Q}
$$

for all vectors $\boldsymbol{u}_{0}:=\left(u_{01}, u_{02}, \ldots, u_{0 Q}\right)=\mathrm{u}_{0} \boldsymbol{e}_{r}$, where $\mathrm{u}_{0} \in \mathbb{C}$ is an $O(1)$ constant and $\boldsymbol{e}_{r}=\left(e_{r 1}, e_{r 2}, \ldots, e_{r Q}\right) \in$ $\left\{\boldsymbol{v} \in \mathbb{C}^{Q}:\|\boldsymbol{v}\|=1\right\}$ is the unit eigenvector of (71) i.e. (94) corresponding to $\tau_{r}$. By following the analysis outlined in Section 4.4, we consequently find that

$$
\begin{aligned}
& \tilde{u}_{0}(\boldsymbol{x})=\mathrm{u}_{0} \sum_{q} e_{r q} \tilde{\varphi}_{n q}^{\boldsymbol{a}}(\boldsymbol{x}), \\
& \tilde{u}_{1}(\boldsymbol{x})=\mathrm{u}_{0} \sum_{q} e_{r q} \boldsymbol{\chi}_{q}^{(1)}(\boldsymbol{x}) \cdot i \hat{\boldsymbol{k}}+\sum_{q} u_{1 q} \tilde{\varphi}_{n q}^{\boldsymbol{a}}(\boldsymbol{x}),
\end{aligned}
$$

where $\boldsymbol{\chi}_{q}^{(1)}$ solve (82). In light of the leading-order solution along $\boldsymbol{e}_{r}$ given by (97), we are interested only in the correction vectors $\boldsymbol{u}_{1}:=\left(u_{11}, u_{12}, \ldots, u_{1 Q}\right)$ satisfying the constraint

$$
\sum_{q} e_{r q} u_{1 q}=0
$$

Considering next the $O(1)$ contribution to (63), we account for residual (95) and integrate $\langle(92)\rangle_{\boldsymbol{a}}^{p \varphi}$ with $\hat{\omega}=0$ by parts to obtain the effective equations

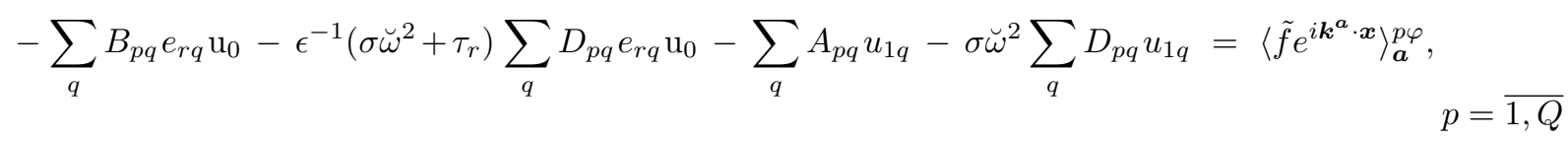

which, together with (98), provide a system of $Q+1$ equations for the coefficients $\mathrm{u}_{0}$ and $u_{1 q}(q=\overline{1, Q})$.

Thanks to (96)-(97), the Bloch-wave solution (26) in this case becomes

$$
\begin{aligned}
\tilde{u}(\boldsymbol{x}) & =e^{-i \boldsymbol{k}^{\boldsymbol{a}} \cdot \boldsymbol{x}}\left(\epsilon^{-2} \tilde{u}_{0}(\boldsymbol{x})+\epsilon^{-1} \tilde{u}_{1}(\boldsymbol{x})\right)+O(1) \\
& =e^{-i \boldsymbol{k}^{\boldsymbol{a}} \cdot \boldsymbol{x}} \sum_{q}\left[\mathrm{u}_{0} e_{r q}\left(\epsilon^{-2} \tilde{\varphi}_{n q}^{\boldsymbol{a}}(\boldsymbol{x})+\epsilon^{-1} \boldsymbol{\chi}_{q}^{(1)}(\boldsymbol{x}) \cdot i \hat{\boldsymbol{k}}\right)+\epsilon^{-1} u_{1 q} \tilde{\varphi}_{n q}^{\boldsymbol{a}}(\boldsymbol{x})\right]+O(1),
\end{aligned}
$$

that features an $O\left(\epsilon^{-2}\right)$ contribution of the solution branch closest to $\left(\boldsymbol{k}^{\boldsymbol{a}}+\epsilon \hat{\boldsymbol{k}}, \tilde{\lambda}_{n}^{\boldsymbol{a}}+\epsilon \sigma \breve{\omega}^{2}\right)$, and an $O\left(\epsilon^{-1}\right)$ contribution of the remaining branches in the cluster. A similar analysis, omitted here for brevity, can be pursued in situations when the coefficient matrix $A_{p q}$ is of partial rank.

\section{Clusters of nearby eigenvalues}

Up until now, our focus was on the "mean" wave motion in a neighborhood of $\left(\boldsymbol{k}^{\boldsymbol{a}}, \tilde{\lambda}_{n}^{\boldsymbol{a}}\right)$, where the eigenvalue $\tilde{\lambda}_{n}^{a}$ is allowed to be either isolated or repeated. An implicit hypothesis in the foregoing analysis, however, is that the distances between $\tilde{\lambda}_{n}^{a}$ and its nearest neighbors are separated from zero. We next aim to relax this restriction by considering a cluster of $Q$ nearby eigenvalues $\tilde{\lambda}_{j}^{a}, j=\overline{n-n_{a}, n+n_{b}}$ that are separated by $O(\epsilon)$ for some $0<\epsilon \ll 1$.

Remark 24. We denote the above cluster by $\tilde{\lambda}_{j(q)}^{a}(q=\overline{1, Q})$, where the mapping $j(q):[1, Q] \rightarrow\left[n-n_{a}, n+n_{b}\right]$ is bijective, and we let $\tilde{\phi}_{n q}^{a}$ be the eigenfunctions corresponding respectively to $\tilde{\lambda}_{j(q)}^{a}$. 


\subsection{Eigenfunction basis}

In line with the above description, we consider a set of nearby eigenvalues $\tilde{\lambda}_{j(q)}^{a}$ given by

$$
\tilde{\lambda}_{j(q)}^{a}=\tilde{\lambda}_{n}^{a}-\epsilon \gamma_{q}, \quad \gamma_{q} \lesseqgtr 0, \quad q=\overline{1, Q} .
$$

where, for definiteness, $\epsilon$ quantifies the (small) relative eigenvalue spread of the cluster:

$$
\epsilon=\frac{1}{2 \tilde{\lambda}_{n}^{a}}\left(\tilde{\lambda}_{j(Q)}^{a}-\tilde{\lambda}_{j(1)}^{a}\right)
$$

Assumption 2. We allow for $\tilde{\lambda}_{n}^{a}$ to have multiplicity $1 \leqslant Q^{\prime}<Q$, and we adopt the mapping $j(q)$ so that $\gamma_{q}=0$ for $q=\overline{1, Q^{\prime}}$. All other eigenvalues $\tilde{\lambda}_{j(q)}^{a}\left(q>Q^{\prime}\right)$ are assumed to be isolated.

From (99), one finds that the eigenfunctions $\tilde{\phi}_{n q}^{\boldsymbol{a}} \in H_{p}^{1}(Y)$ corresponding to $\tilde{\lambda}_{j(q)}^{a}$ solve

$$
\begin{aligned}
& -\left(\nabla+i \boldsymbol{k}^{\boldsymbol{a}}\right) \cdot\left(G\left(\nabla+i \boldsymbol{k}^{\boldsymbol{a}}\right) \tilde{\phi}_{n q}^{\boldsymbol{a}}\right)=\left(\tilde{\lambda}_{n}^{a}-\epsilon \gamma_{q}\right) \rho \tilde{\phi}_{n q}^{a} \quad \text { in } Y, \\
& \llbracket \tilde{\phi}_{n q}^{a} \rrbracket+\kappa^{-1} t_{\nu}^{\boldsymbol{k}^{\boldsymbol{a}}}\left[\tilde{\phi}_{n q}^{\boldsymbol{a}}, \tilde{\phi}_{n q}^{\boldsymbol{a}}\right]=0 \quad \text { on } \Gamma,\left.\quad 1 t_{\boldsymbol{n}}^{\boldsymbol{k}^{\boldsymbol{a}}}\left[\tilde{\phi}_{n q}^{\boldsymbol{a}}, \tilde{\phi}_{n q}^{\boldsymbol{a}}\right]\right|_{j} ^{+}=0 \quad \text { on } \partial Y \text {. }
\end{aligned}
$$

Following the earlier analysis, we introduce the real-valued eigenfunctions $\tilde{\varphi}_{n q}^{a} \in H_{p}^{1}\left(Y_{\boldsymbol{a}}\right)$ that are (i) orthogonal in $L_{\rho}^{2}\left(Y_{\boldsymbol{a}}\right)$; (ii) related to $\tilde{\phi}_{n q}^{a}$ via (15), and (iii) solve

$$
\begin{gathered}
-\nabla \cdot\left(G \nabla \tilde{\varphi}_{n q}^{\boldsymbol{a}}\right)=\left(\tilde{\lambda}_{n}^{\boldsymbol{a}}-\epsilon \gamma_{q}\right) \rho \tilde{\varphi}_{n q}^{\boldsymbol{a}} \quad \text { in } Y_{\boldsymbol{a}}, \\
\llbracket \tilde{\varphi}_{n q}^{\boldsymbol{a}} \rrbracket+\kappa^{-1} t_{\boldsymbol{\nu}}\left[\tilde{\varphi}_{n q}^{\boldsymbol{a}}\right]=0 \quad \text { on } \Gamma_{\boldsymbol{a}},\left.\quad 1 t_{\boldsymbol{n}}\left[\tilde{\varphi}_{n q}^{\boldsymbol{a}}\right]\right|_{\boldsymbol{a}, j} ^{+}=0 \text { on } \partial Y_{\boldsymbol{a}},
\end{gathered}
$$

Inherently, (101)-(103) suggest the use of $Q$ averaging operators (65) and the "zero-mean" function space (66), which now entails $Q$ projections onto the eigenfunctions featured by nearby dispersion branches.

Remark 25. In contrast to the preceding analysis where $\epsilon$ played the role of a perturbation parameter, $\epsilon$ in (99) is a small constant that signifies, in physical terms, the "width" of the eigenvalue cluster. By letting $\epsilon=o(1)$, we implicitly assume normalization with respect to a suitable dimensional basis such that $\tilde{\lambda}_{j(q)}^{a}-\tilde{\lambda}_{m}^{a} \geqslant O(1)$ for $j=\overline{1, Q}$ and $m \notin\left[n-n_{a}, n+n_{b}\right]$. In this setting, ansatz (26) and the near-apex description (62) can still be deployed as a basis for development of the local approximation (as shown below), noting however that the rigorous asymptotic analysis - as needed - must be pursued by letting $\zeta:=\|\hat{\boldsymbol{k}}\| \rightarrow 0$ while keeping $\epsilon$ small but fixed according to (100).

Remark 26. In light of the above assumptions, it is in particular important to justify the wavenumber scaling $\boldsymbol{k}=\boldsymbol{k}^{\boldsymbol{a}}+\epsilon \hat{\boldsymbol{k}}$ in (62), for we only know that the "width" of the eigenvalue cluster is $O(\epsilon)$. Implicitly, such scaling limits the range of $\boldsymbol{k}-\boldsymbol{k}^{\boldsymbol{a}}$ over which the local approximation applies. Considering the local wave dispersion as a guide, from (62) we consequently obtain the requirement that

$$
\left.\omega^{2}(\hat{\boldsymbol{k}})\right|_{j(q)}-\tilde{\lambda}_{n}^{a}=-\epsilon \gamma_{j(q)}+\left.\epsilon \sigma \breve{\omega}^{2}(\hat{\boldsymbol{k}})\right|_{j(q)}+\left.\epsilon^{2} \sigma \hat{\omega}^{2}(\hat{\boldsymbol{k}})\right|_{j(q)} \leqslant O(\epsilon), \quad q=\overline{1, Q},
$$

where $\left.\omega^{2}(\hat{\boldsymbol{k}})\right|_{j(q)}$ describes locally the $j(q)$ th dispersion branch. From the results in Section 4 (repeated eigenvalues), we find that taking $\boldsymbol{k}=\boldsymbol{k}^{a}+\epsilon \hat{\boldsymbol{k}}$ results, loosely speaking, in the scalings

$$
\sigma \breve{\omega}^{2}(\hat{\boldsymbol{k}})=O\left(\frac{\boldsymbol{\theta}_{p q}^{(0)} \cdot i \hat{\boldsymbol{k}}}{\rho_{p q}^{(0)}}\right) \leqslant O(1), \quad \sigma \hat{\omega}^{2}(\hat{\boldsymbol{k}})=O\left(\frac{\boldsymbol{\mu}_{p q}^{(0)}: \hat{\boldsymbol{k}}^{2}}{\rho_{p q}^{(0)}}\right) \leqslant O(1)
$$

for any $\epsilon=o(1)$ and $\|\hat{\boldsymbol{k}}\|=O(1)$. Assuming that a similar result applies to the case of nearby eigenvalues (as demonstrated below), this justifies the assumed wavenumber scaling. From (105), one may also draw a generic expectation that for dispersion branches in the cluster with smaller "slopes" $\sigma \breve{\omega}^{2}$ and smaller "curvatures" $\sigma \hat{\omega}^{2}$, the local approximation will hold over a wider range of $\hat{\boldsymbol{k}}$; this behavior is indeed observed via numerical simulations, see Section 6. 


\subsection{Leading-order approximation}

We again refer to (24), and we start from the asymptotic expansion (26) of $\tilde{u}$. For clarity of presentation, we consider the interfacial and boundary conditions over $\Gamma, \partial Y, \Gamma_{a}$ and $\partial Y_{\boldsymbol{a}}$ such as those in (25), (102) and (104) only implicitly. From the results in Section 3.5, one finds that the $O\left(\epsilon^{-2}\right)$ contribution to (24) is given by (29). Owing to (103), $\tilde{u}_{0}$ given by (67) satisfies $(29)$ up to the $O(\epsilon)$ residual

$$
\epsilon \rho(\boldsymbol{x}) \sum_{q} \gamma_{q} u_{0 q} \tilde{\varphi}_{n q}^{\boldsymbol{a}}(\boldsymbol{x})
$$

This term carries over to the $O\left(\epsilon^{-1}\right)$ statement of $(24)$, which now reads

$$
-\tilde{\lambda}_{n}^{\boldsymbol{a}} \rho \tilde{u}_{1}-\sigma \breve{\omega}^{2} \rho \tilde{u}_{0}-\nabla \cdot\left(G\left(\nabla \tilde{u}_{1}+i \hat{\boldsymbol{k}} \tilde{u}_{0}\right)\right)-i \hat{\boldsymbol{k}} \cdot\left(G \nabla \tilde{u}_{0}\right)-\rho \sum_{q} \gamma_{q} u_{0 q} \tilde{\varphi}_{n q}^{\boldsymbol{a}}=0 \text { in } Y_{\boldsymbol{a}} .
$$

On integrating $\langle(106)\rangle_{\boldsymbol{a}}^{p \varphi}$ by parts, one can show as in the proof of Lemma 2 that

$$
\sum_{q} A_{p q}^{\gamma} u_{0 q}-\tau \sum_{q} D_{p q} u_{0 q}=0, \quad p=\overline{1, Q}
$$

up to respective $O(\epsilon)$ residuals

$$
\epsilon \gamma_{p}\left\langle\rho \tilde{u}_{1}\right\rangle_{\boldsymbol{a}}^{p \varphi}, \quad p=\overline{1, Q}
$$

where

$$
A_{p q}^{\gamma}=\boldsymbol{\theta}_{p q}^{(0)} \cdot i \hat{\boldsymbol{k}}+\gamma_{q} D_{p q}, \quad D_{p q}=\delta_{p q} \rho_{q}^{(0)}, \quad \tau=-\sigma \breve{\omega}^{2},
$$

with $\rho_{p}^{(0)}$ and $\boldsymbol{\theta}_{p q}^{(0)}$ given by (70). Whenever possible, we seek a non-trivial solution to (107).

Remark 27. Since $A_{p q}^{\gamma}$ is Hermitian and $D_{p q}$ is diagonal positive definite, the eigenvalues $\tau$ of (107) are real, and the eigenvectors corresponding to distinct eigenvalues are orthogonal.

\subsubsection{Effective model for full-rank $A_{p q}^{\gamma}$}

When $\operatorname{rank}\left(A_{p q}^{\gamma}\right)=Q$, generalized eigenvalue problem (107) has no zero eigenvalues. Accordingly, the scaling framework (23) with $\breve{\omega}=0$ does not cater for a non-trivial solution of (107); we thus keep $\breve{\omega} \neq 0$ and set $u_{0 q}=0$ $(q=\overline{1, Q})$, by which the $O\left(\epsilon^{-1}\right)$ statement of (106) reduces to (73). Thanks to (103), one can take $\tilde{u}_{1}(\boldsymbol{x})$ as in (74), which satisfies (73) up to the $O(\epsilon)$ residual

$$
\epsilon \rho(\boldsymbol{x}) \sum_{q} \gamma_{q} u_{1 q} \tilde{\varphi}_{n q}^{\boldsymbol{a}}(\boldsymbol{x})
$$

that carries over to the $O(1)$ statement of $(24)$, namely

$$
-\tilde{\lambda}_{n}^{\boldsymbol{a}} \rho \tilde{u}_{2}-\nabla \cdot\left(G\left(\nabla \tilde{u}_{2}+i \hat{\boldsymbol{k}} \tilde{u}_{1}\right)\right)-i \hat{\boldsymbol{k}} \cdot\left(G \nabla \tilde{u}_{1}\right)-\sigma \breve{\omega}^{2} \rho \tilde{u}_{1}-\rho \sum_{q} \gamma_{q} u_{1 q} \tilde{\varphi}_{n q}^{\boldsymbol{a}}=\tilde{f} e^{i \boldsymbol{k}^{\boldsymbol{a}} \cdot \boldsymbol{x}} \quad \text { in } Y_{\boldsymbol{a}} .
$$

To compute $u_{1 q} \in \mathbb{C}$ in $(74)$, we integrate $\langle(110)\rangle_{\boldsymbol{a}}^{p \varphi}(p \in \overline{1, Q})$ by parts which yields

$$
-\sum_{q} A_{p q}^{\gamma} u_{1 q}-\sigma \breve{\omega}^{2} \sum_{q} D_{p q} u_{1 q}=\left\langle\tilde{f} e^{i \boldsymbol{k}^{\boldsymbol{a}} \cdot \boldsymbol{x}}\right\rangle_{\boldsymbol{a}}^{p \varphi}, \quad p=\overline{1, Q}
$$

up to $O(\epsilon)$ residuals, where $A_{p q}^{\gamma}$ and $D_{p q}$ are given by (108), and $\sigma \breve{\omega}^{2}=\epsilon^{-1}\left(\omega^{2}-\tilde{\lambda}_{n}^{a}\right)$ according to (23). By Remark 27, we find that the generalized eigenvalue problem behind (111) has $Q$ real eigenvalues, hereon denoted by $\tau_{r}(r=\overline{1, Q})$, and we assume $-\sigma \breve{\omega}^{2} \neq \tau_{r}$ when solving (111). Since $A_{p q}^{\gamma}$ is not antisymmetric, however, the eigenvalues $\tau_{r}$ of (111) will generally not appear in " \pm " pairs as in the case of repeated eigenvalues, see Section 4.3.

Remark 28. The case of trivial $A_{p q}^{\gamma}=A_{p q}+\gamma_{q} D_{p q}$ is not relevant to the analysis for it would require that each $A_{p q} \in \mathbb{I}$ and $\gamma_{q} D_{p q} \in \mathbb{R}$ vanish identically - a premise that yields $\gamma_{p}=0(p=\overline{1, Q})$ and thus precludes the existence of nearby eigenvalues. 


\subsubsection{Effective model for $\operatorname{rank}\left(A_{p q}^{\gamma}\right)=Q-1$}

To illustrate the analysis of clusters featuring partial-rank $A_{p q}$, we recall Section 4.5 and we focus on situations where $A_{p q}=\Sigma_{p q}$ according to (86) with $N=N_{0}=1$, i.e. $\operatorname{rank}\left(A_{p q}^{\gamma}\right)=Q-1$. Specifically, we let

$$
A_{1 p}^{\gamma}=A_{p 1}^{\gamma}=0, \quad p=\overline{1, Q}
$$

which implies $\gamma_{1}=0$, see also Assumption 2. More general cluster situations where $N>N_{0}>0$ can be studied in a similar way.

Lemma 7. Let $\operatorname{rank}\left(A_{p q}^{\gamma}\right)=Q-1$, and let the direction $\hat{\boldsymbol{k}} /\|\hat{\boldsymbol{k}}\|$ be fixed. If (112) holds for some $\kappa=\|\hat{\boldsymbol{k}}\|>0$, then it holds for all $\kappa>0$.

Proof. From (108), we see that

$$
A_{p q}^{\gamma}=\gamma_{q} D_{p q}+\left(\boldsymbol{\theta}_{p q}^{(0)} \cdot \kappa^{-1} \hat{\boldsymbol{k}}\right) i \kappa, \quad p, q \in \overline{1, Q}
$$

where $\gamma_{q} D_{p q} \in \mathbb{R}$ and $\left(\boldsymbol{\theta}_{p q}^{(0)} \cdot \kappa^{-1} \hat{\boldsymbol{k}}\right) \in \mathbb{R}$ are independent of $\kappa$. If (112) holds for some $\kappa>0$, then each of these two terms must vanish identically for $(p, q) \rightarrow(1, p)$ and $(p, q) \rightarrow(p, 1)$.

Thanks to (112), (107) becomes an identity (to the leading order) if: (a) $\tau=-\sigma \breve{\omega}^{2}=O(\epsilon$ ), and (b) $u_{0 q}=\mathrm{u}_{0} \delta_{q 1}$ i.e.

$$
\tilde{u}_{0}(\boldsymbol{x})=\mathrm{u}_{0} \tilde{\varphi}_{n 1}^{a}(\boldsymbol{x}),
$$

for some constant $\mathrm{u}_{0} \in \mathbb{C}$, see also Section 4.5 for related discussion. On recalling that $\gamma_{1}=0$, this result yields $\gamma_{q} u_{0 q}=0(q=\overline{1, Q})$; accordingly we see that the $O\left(\epsilon^{-1}\right)$ field equation (106) is solved by

$$
\tilde{u}_{1}(\boldsymbol{x})=\mathrm{u}_{0} \boldsymbol{\chi}_{1}^{(1)}(\boldsymbol{x}) \cdot i \hat{\boldsymbol{k}}+\sum_{q} u_{1 q} \tilde{\varphi}_{n q}^{\boldsymbol{a}}(\boldsymbol{x}),
$$

up to an $O(\epsilon)$ residual given by (109). Here $u_{1 q} \in \mathbb{C}$ are constants, and $\boldsymbol{\chi}_{1}^{(1)} \in\left(H_{p 0}^{1 \boldsymbol{a}}\left(Y_{\boldsymbol{a}}\right)\right)^{d}$ uniquely solves

$$
\tilde{\lambda}_{n}^{a} \rho \boldsymbol{\chi}_{1}^{(1)}+\nabla \cdot\left(G\left(\nabla \boldsymbol{\chi}_{1}^{(1)}+\boldsymbol{I} \tilde{\varphi}_{n 1}^{\boldsymbol{a}}\right)\right)+G \nabla \tilde{\varphi}_{n 1}^{\boldsymbol{a}}-\sum_{r} \frac{1}{\rho_{r}^{(0)}} \boldsymbol{\theta}_{r 1}^{(0)} \rho \tilde{\varphi}_{n r}^{\boldsymbol{a}}=0 \quad \text { in } Y_{\boldsymbol{a}}
$$

subject to relevant interfacial and boundary conditions (see (82)), noting that $\tilde{\lambda}_{j(1)}^{a}=\tilde{\lambda}_{n}^{a}-\epsilon \gamma_{1}=\tilde{\lambda}_{n}^{a}$. In this setting the $O(1)$ statement of (24), which accounts for residual (109), becomes

$$
\begin{aligned}
& -\tilde{\lambda}_{n}^{\boldsymbol{a}} \rho \tilde{u}_{2}-\nabla \cdot\left(G\left(\nabla \tilde{u}_{2}+i \hat{\boldsymbol{k}} \tilde{u}_{1}\right)\right)-i \hat{\boldsymbol{k}} \cdot\left(G\left(\nabla \tilde{u}_{1}+i \hat{\boldsymbol{k}} \tilde{u}_{0}\right)\right)-\sigma \hat{\omega}^{2} \rho \tilde{u}_{0} \\
& -\rho \sum_{q} \gamma_{q} u_{1 q} \tilde{\varphi}_{n q}^{\boldsymbol{a}}=\tilde{f} e^{i \boldsymbol{k}^{\boldsymbol{a}} \cdot \boldsymbol{x}} \text { in } Y_{\boldsymbol{a}} .
\end{aligned}
$$

On integrating $\langle(114)\rangle_{\boldsymbol{a}}^{1 \varphi}$ by parts, we find that

$$
-\left(\boldsymbol{\mu}_{11}^{(0)}:(i \hat{\boldsymbol{k}})^{2}+\sigma \hat{\omega}^{2} \rho_{1}^{(0)}\right) \mathrm{u}_{0}=\left\langle\tilde{f} e^{i \boldsymbol{k}^{\boldsymbol{a}} \cdot \boldsymbol{x}}\right\rangle_{\boldsymbol{a}}^{1 \varphi},
$$

where $\rho_{1}^{(0)}$ and $\boldsymbol{\mu}_{11}^{(0)}$ are given respectively by (70) and (84). Note that (115) is formally the same as (91) with $N=N_{0}=1$, catering for the case of repeated eigenvalues.

To identify the remaining $Q-1$ leading-order projections in terms of $u_{1 q}(q=\overline{2, Q})$, we next integrate (114) as $\langle\cdot\rangle_{\boldsymbol{a}}^{p \varphi}$ for $p=\overline{2, Q}$, see Section 4.5 with $N=N_{0}=1$, to obtain the linear system

$$
-\sum_{q>1} A_{p q}^{\gamma} u_{1 q}=\left\langle\tilde{f} e^{i \boldsymbol{k}^{a} \cdot \boldsymbol{x}}\right\rangle_{\boldsymbol{a}}^{p \varphi}+\mathrm{u}_{0} \boldsymbol{\mu}_{p 1}^{(0)}:(i \hat{\boldsymbol{k}})^{2}
$$

where $\boldsymbol{\mu}_{p 1}^{(0)}$ and $\boldsymbol{\chi}_{1}^{(1)}$ are given respectively by (84) and (113). By the assumption on $A_{p q}^{\gamma}$, this equation is solvable for $u_{1 q}, q=\overline{2, Q}$. 


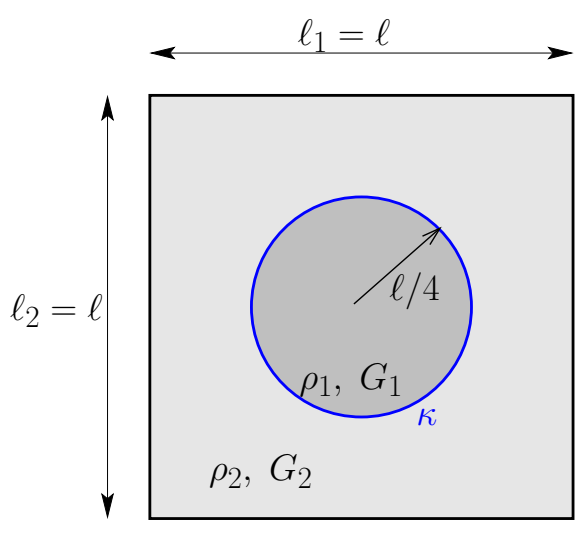

(a) Periodicity cell $Y$

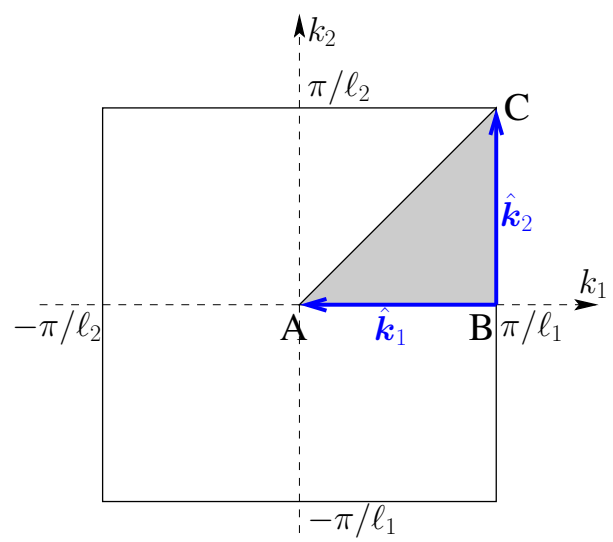

(b) First Brillouin zone, with wavenumber perturbation directions $\hat{\boldsymbol{k}}_{1}, \hat{\boldsymbol{k}}_{2}$ about apex B

Figure 2: Periodic medium with circular compliant interface.

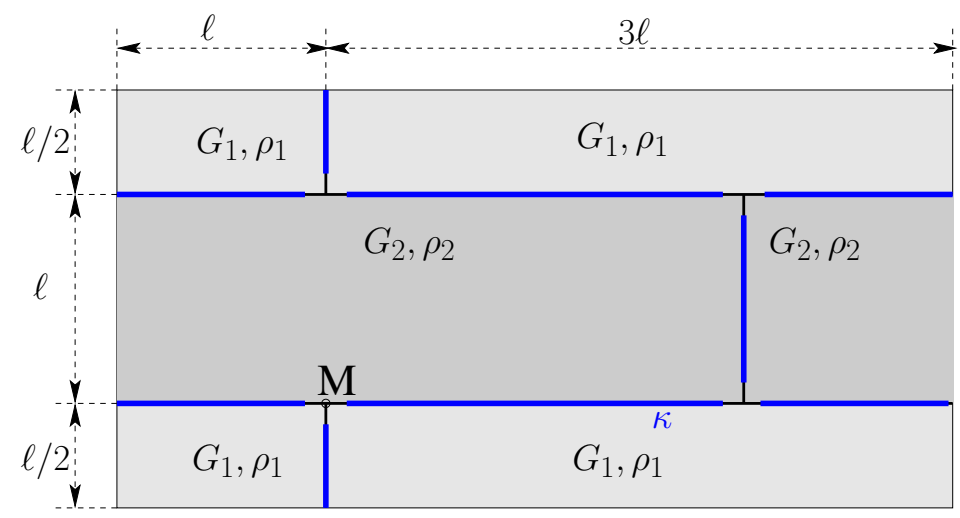

Figure 3: Periodicity cell $Y$ for medium made of staggered bricks separated by compliant interfaces (in blue). The bricks are bonded by ligaments near the "triple points" such as M.

Remark 29. We observe a remarkable similarity between (116) and its repeated-eigenvalues counterpart (93) with $N=1$, where the only formal difference is the appearance of a diagonally-perturbed matrix, $A_{p q}^{\gamma}=$ $A_{p q}+\gamma_{q} D_{p q}$, in lieu of $A_{p q}$. The same comment applies to (111) and (77). As will be seen shortly, the perturbation contained in $A_{p q}^{\gamma}$ has the effect of "blunting" the conical dispersion surfaces. On revisiting the foregoing analysis with $\sigma \breve{\omega}^{2}=O(1)$ in lieu of $\sigma \breve{\omega}^{2}=O(\epsilon)$, one would find (assuming that the driving frequency is not "close" to any branch in the cluster in the sense of Section 4.6) the germane projections to behave $O\left(\epsilon^{-1}\right)$, as defined by $u_{1 q}$ solving (116) for $p=\overline{1, Q}$.

Analogous to the case of repeated eigenvalues, the "full" effective model when $\operatorname{rank}\left(A_{p q}^{\gamma}\right)=Q-1$ is given by $\mathrm{u}_{0}$ solving (115), and $u_{1 q}(q=\overline{2, Q})$ solving (116). In terms of the implied dispersion within the cluster, we further see that (115)-(116) describe a single parabola accompanied by $Q-1$ "cones". Note that when computing the "conical" dispersion due to (116), one must set $\mathrm{u}_{0}=0$ because (in the absence of a source term) its nontrivial values require by (115) that $\omega^{2}-\tilde{\lambda}_{n}^{a}=O\left(\epsilon^{2}\right)$, whereas (116) assumes $\omega^{2}-\tilde{\lambda}_{n}^{a}=O(\epsilon)$.

\section{Numerical results}

We consider two examples of periodic media, whose unit cell of periodicity, $Y=\left(0, \ell_{1}\right) \times\left(0, \ell_{2}\right) \subset \mathbb{R}^{2}$, features compliant interfaces. The disk configuration involves a square periodicity cell $\left(\ell_{1}=\ell_{2}=\ell\right)$ containing two homogeneous materials separated by a centric circular interface of radius $\ell / 4$ (Fig. 2). For the brickwall example, evocative of a masonry featuring staggered rectangular bricks of dimensions $2 \ell \times \ell$ (Fig. 3), we 
assume $\ell_{1}=4 \ell$ and $\ell_{2}=2 \ell$. Here the interfaces are assumed to be compliant outside of short ligaments near the junction points (such as the point $\mathrm{M}$ in Fig. 3), along which the bricks are perfectly bonded to each other.

In what follows, the featured asymptotic approximations of forced motions, dispersion branches or eigenfunctions near apexes A,B,C of the first Brillouin zone (defined as in Fig. 2b) are compared with respective reference solutions obtained by numerically solving the eigenvalue or forced-vibration problems at perturbed wavenumbers and frequencies. These numerical computations, as well as the evaluations of cell functions and effective coefficients of homogenization, are performed using the XLife++ finite element library [17]. To accurately evaluate the eigenvalues at apexes (for proper distinction between repeated eigenvalues and dense clusters thereof) and reference dispersion diagrams, the first 30 eigenvalues of problem (9) are computed at all discrete wavenumbers produced by uniformly dividing each side of the Brillouin triangle ABC (Fig. 2b) into 200 segments via discretization of $Y$ into 2216 (disk) or 5690 (brick wall) fourth-order triangular finite elements. All eigenvalue problems are formulated in terms of the factorized version $\tilde{\varphi}_{n, \epsilon}^{a}(\boldsymbol{x}):=e^{-i \boldsymbol{k}^{a} \cdot \boldsymbol{x}} \tilde{\phi}_{n, \epsilon}^{\boldsymbol{a}}(\boldsymbol{x})$ of eigenfunctions $\tilde{\phi}_{n, \epsilon}^{a}$ for perturbed frequencies and wavenumbers (see (15)).

\subsection{Asymptotic approximation of dispersion branches and eigenfunctions}

We begin by examining the local approximation of dispersion branches stemming from the analysis presented in Sections 3-5. Figs. 4 and 5 show, for the disk configuration endowed respectively with $\rho_{1}=2 \rho_{2}, \kappa=1$ and $\rho_{1}=5 \rho_{2}, \kappa=10$, the local asymptotic approximation of the first nine dispersion branches emanating from apex points A, B and C, superimposed on their numerically computed counterparts (for completeness, we note that the separation from the ninth and tenth dispersion branches in the spectral neighborhood of the apexes A, B, C is sufficient to avoid involving the latter in nearby clusters for this example). The featured approximations are plotted for branches emanating from isolated, repeated (with $Q=2$ or 3 ) or clustered apex eigenvalues (with $Q=2,3$ or 4). To aid the interpretation of Figs. 4 and 5 , the breakdown of relevant apex eigenvalues into isolated, repeated and cluster configurations is provided in Table 1 . The asymptotic surrogates of dispersion branches (or sets thereof) provide good approximations of the actual dispersion branches in spectral neighborhoods of the apexes spanning about one-fourth of each Brillouin triangle side.

The most interesting eigenvalue configuration featured in the above dispersion diagrams is the four-fold cluster $(6-9)_{\mathrm{N}}$ at apex $\mathrm{C}$ in Fig. 5. A blow-up of this neighborhood is shown in Fig. 6a, together with the affiliated asymptotic approximations. For completeness, we note that the apparent crossing of branches 7 and 8

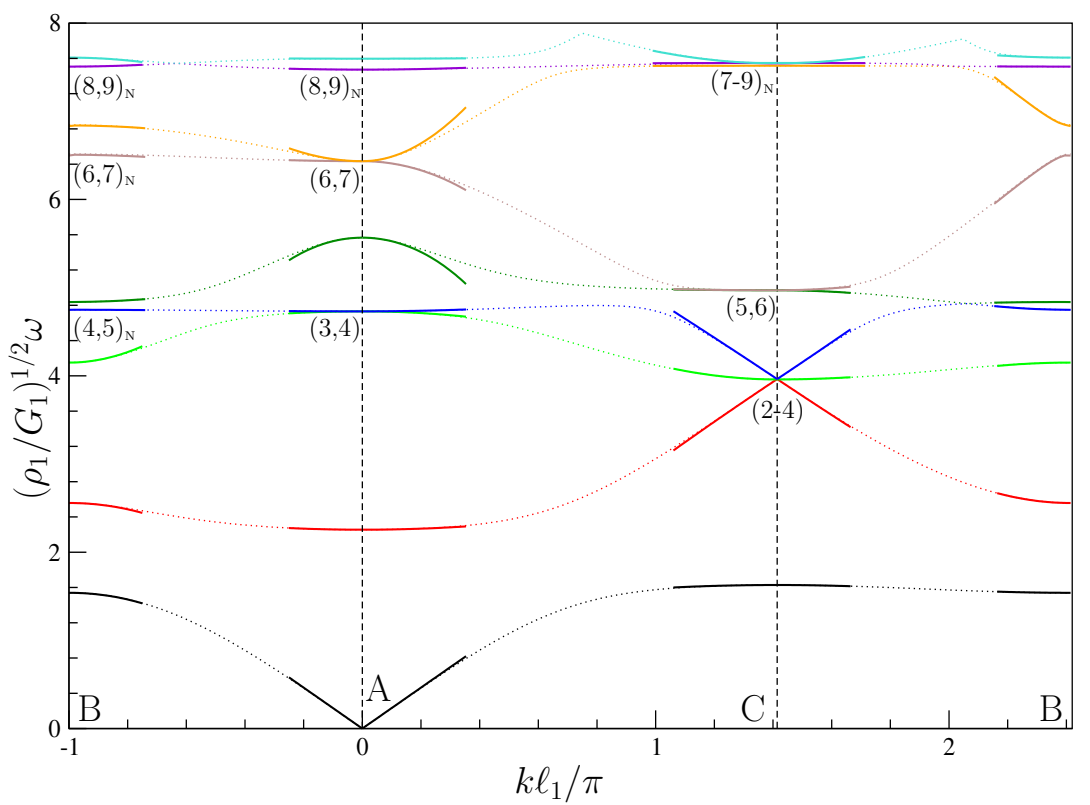

Figure 4: Disk $\left(\rho_{2}=2 \rho_{1}, \kappa=1\right)$ : first nine dispersion branches. Reference branches (numerically computed using XLife $++[17]$ ) are shown as dotted lines, while solid lines track asymptotic approximations near apexes A,B,C. 


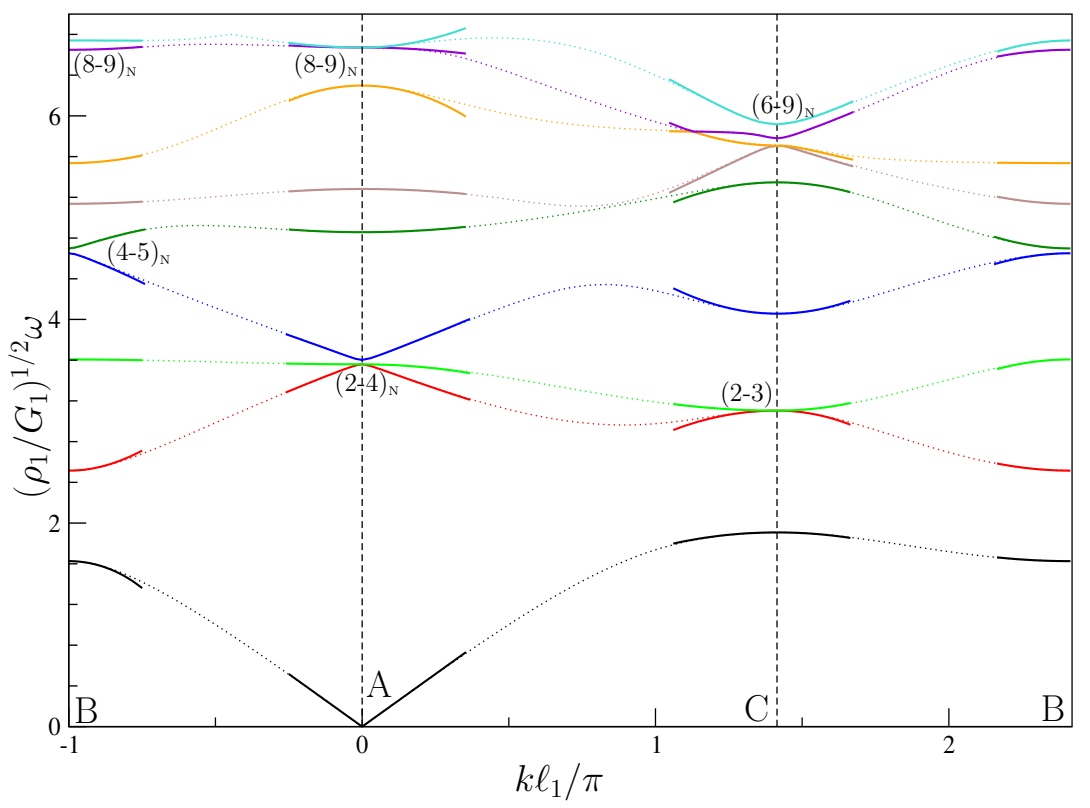

Figure 5: Disk $\left(\rho_{2}=5 \rho_{1}, \kappa=10\right.$ ): first nine dispersion branches. Reference branches (numerically computed using XLife $++[17]$ ) are shown as dotted lines, while solid lines track asymptotic approximations near apexes A,B,C.

\begin{tabular}{|c|l|}
\hline Apex & Eigenvalue status \\
\hline A & $12(3,4) 5(6,7)(8,9)_{\mathrm{N}}$ \\
B & $123(4,5)_{\mathrm{N}}(67)_{\mathrm{N}}(8,9)_{\mathrm{N}}$ \\
C & $1(2,3,4)(5,6)(7,8,9)_{\mathrm{N}}$ \\
\hline
\end{tabular}

(a) Case $\rho_{2}=2 \rho_{1}, \kappa=1$ (corresponding to Fig. 4)

\begin{tabular}{|c|l|}
\hline Apex & Eigenvalue status \\
\hline A & $1(2,3,4)_{\mathrm{N}} 567(8,9)_{\mathrm{N}}$ \\
B & $123(4,5)_{\mathrm{N}} 67(8,9)_{\mathrm{N}}$ \\
C & $1(2,3) 45(6,7,8,9)_{\mathrm{N}}$ \\
\hline
\end{tabular}

(b) Case $\rho_{2}=5 \rho_{1}, \kappa=10$ (corresponding to Fig. 5)

Table 1: Breakdown of the apex eigenvalues featured in Figs. 4 (left table) and 5 (right table) into isolated, repeated and nearby clusters. Groupings within parentheses indicate nearby clusters (if appended with a "N" subscript) or repeated eigenvalues; numbers outside parentheses indicate isolated eigenvalues.

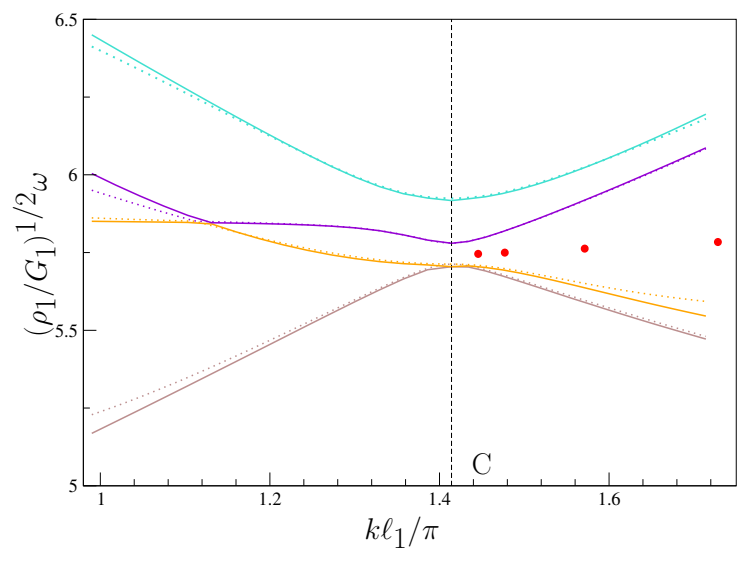

(a) Disk example $\left(\rho_{2}=5 \rho_{1}, \kappa=10\right)$, dispersion branch cluster (6$9)_{\mathrm{N}}$ near apex $\mathrm{C}$ (with red dots used for Table 3 ).

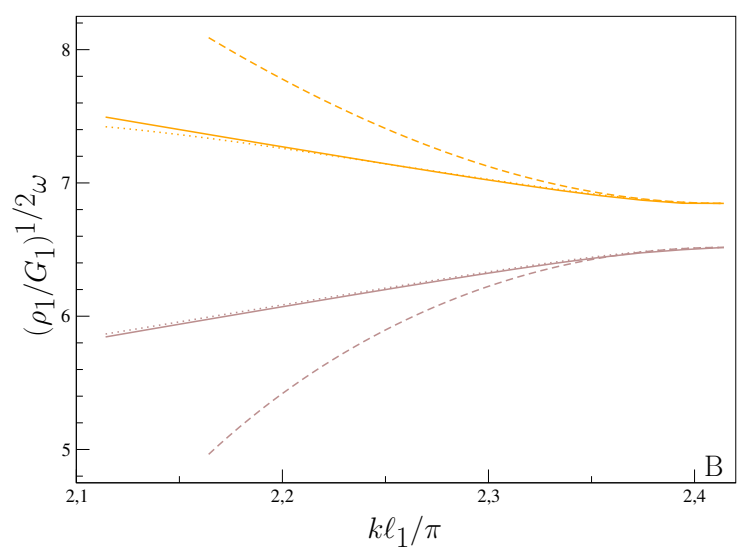

(b) Disk example $\left(\rho_{2}=2 \rho_{1}, \kappa=1\right)$ : dispersion branch cluster $(6,7)_{\mathrm{N}}$ near apex B.

Figure 6: Blow-up of the selected branch clusters. Numerically-computed reference branches are shown as dotted lines, while their local asymptotic approximations (cluster model) are indicated by solid lines. Fig. $6 \mathrm{~b}$ also shows the asymptotic approximations given by the isolated-eigenvalue model (dashed lines). 
to the left of the apex can be either an actual crossing or an avoided crossing [18] (level repulsion associated with a close exceptional point in the complex wavenumber space); in the present case, computational "zoomins" of branches 7 and 8 near that point (not shown) suggest an actual crossing. The utility of the "nearby" asymptotic model is further exemplified by the results for cluster $(6,7)_{\mathrm{N}}$ at apex B in Fig. 4: the improvement brought by the nearby model, relative to the isolated-eigenvalue framework of Sec. 3, is evident from the detail shown in Fig. 6b.

\subsection{Asymptotic approximation of eigenfunctions}

We next compare the asymptotic approximation of featured eigenfunctions with their reference counterparts, considering the brickwall configuration with $10 \%$ ligament length and examining the frequency and wavenumber perturbations about an isolated eigenvalue $\tilde{\lambda}_{n}^{a}(n=25)$ at apex C. Considering the frequency and wavenumber perturbations (23), the (numerically computed) reference eigenfunction $\tilde{\varphi}_{n, \epsilon}^{a, \text { ref }}$ solves

$$
\begin{gathered}
-(\nabla+\epsilon \hat{\mathrm{k}}) \cdot\left(G(\nabla+\epsilon \mathrm{i} \hat{\boldsymbol{k}}) \tilde{\varphi}_{n, \epsilon}^{\boldsymbol{a}, \mathrm{ref}}\right)=\left(\tilde{\lambda}_{n}^{\boldsymbol{a}}+\epsilon^{2} \sigma \hat{\omega}^{2}\right) \rho \tilde{\varphi}_{n, \epsilon}^{\boldsymbol{a}, \mathrm{ref}} \quad \text { in } Y_{\boldsymbol{a}}, \\
\llbracket \tilde{\varphi}_{n}^{\boldsymbol{a}} \rrbracket+\kappa^{-1} t_{\boldsymbol{\nu}}\left[\tilde{\varphi}_{n}^{\boldsymbol{a}}\right]=0 \quad \text { on } \Gamma_{\boldsymbol{a}},\left.\quad 1 t_{\boldsymbol{n}}\left[\tilde{\varphi}_{n}^{\boldsymbol{a}}\right]\right|_{\boldsymbol{a}, j} ^{+}=0 \quad \text { on } \partial Y_{\boldsymbol{a}},
\end{gathered}
$$

i.e. the perturbed counterpart of problem (18)-(19), while its second-order asymptotic approximation $\tilde{\varphi}_{n, \epsilon}^{\boldsymbol{a} \text {,asy }}$ is found (see Appendix, Sec. 7 for proof) to be given by

$$
\tilde{\varphi}_{n, \epsilon}^{\boldsymbol{a}, \text { ref }}=\tilde{\varphi}_{n, \epsilon}^{\boldsymbol{a}, \text { asy }}+o\left(\epsilon^{2}\right) \quad \text { with } \quad \tilde{\varphi}_{n, \epsilon}^{\boldsymbol{a}, \text { asy }}:=C\left(1-\frac{1}{2} \epsilon^{2}\left\|\mathrm{i} \hat{\boldsymbol{k}} \cdot \boldsymbol{\chi}^{(1)}\right\|_{L^{2}(Y)}^{2}\right)\left(\tilde{\varphi}_{n}^{\boldsymbol{a}}+\epsilon \mathrm{i} \hat{\boldsymbol{k}} \cdot \boldsymbol{\chi}^{(1)}+\epsilon^{2}(\mathrm{i} \hat{\boldsymbol{k}})^{2}: \boldsymbol{\chi}^{(2)}\right) .
$$

The normalization convention (11), verified up to order $O\left(\epsilon^{2}\right)$ by $\tilde{\varphi}_{n, \epsilon}^{\boldsymbol{a} \text {,asy }}$ as given above, still allows any unitnorm complex factor $C$ in (117), which is then selected by requiring that $\left\|\tilde{\varphi}_{n, \epsilon}^{\boldsymbol{a}, \text { asy }}-\tilde{\varphi}_{n}^{\boldsymbol{a}}\right\|_{L^{2}(Y)}$ be smallest; the same convention is of course applied to $\tilde{\varphi}_{n, \epsilon}^{\boldsymbol{a} \text {,ref }}$. Figs. 7 compare $\tilde{\varphi}_{n, \epsilon}^{\boldsymbol{a} \text {,asy }}$ and $\tilde{\varphi}_{n, \epsilon}^{\boldsymbol{a} \text {,ref }}$ for wavenumber perturbations (23) with $\epsilon=0.25$ and $\hat{\boldsymbol{k}}=\hat{\boldsymbol{k}}_{1}$ or $\hat{\boldsymbol{k}}=\hat{\boldsymbol{k}}_{2}\left(\hat{\boldsymbol{k}}_{1,2}\right.$ being defined as in Fig. 7f). The single-cell computational reduction of the multi-cell setting described in Sec. 3.3 entails (for this case) anti-periodic conditions on the whole $\partial Y$, which are apparent in the featured real and imaginary parts of $\tilde{\varphi}_{n}^{a}, \tilde{\varphi}_{n, \epsilon}^{a, \text { ref }}$ and $\tilde{\varphi}_{n, \epsilon}^{\boldsymbol{a} \text {,asy }}$. A very good visual agreement between $\tilde{\varphi}_{n, \epsilon}^{\boldsymbol{a} \text {,asy }}$ and $\tilde{\varphi}_{n, \epsilon}^{\boldsymbol{a}, \mathrm{rref}}$ is clearly observed. That agreement is further assessed in Fig. 8, where the relative differences (in $L^{2}(Y)$ norm) between $\tilde{\varphi}_{n, \epsilon}^{a, \text { ref }}$ and their zeroth-, first- and second-order asymptotic approximations contained in (117) are plotted against $\epsilon$. In particular, those differences are seen to exhibit the expected scaling order w.r.t. $\epsilon$. The same level of agreement between $\tilde{\varphi}_{n, \epsilon}^{\boldsymbol{a} \text {,asy }}$ and $\tilde{\varphi}_{n, \epsilon}^{\boldsymbol{a} \text {,ref }}$ is obtained for the disk case, shown in Fig. 9 (computations made at apex $\mathrm{C}$ with $\rho_{1}=2 \rho_{2}$ and $\kappa=1$ ).

\subsection{Asymptotic approximation of mean motion near apex eigenfrequencies}

We next assess the asymptotic approximation of the effective motion $\langle\tilde{u}\rangle_{\boldsymbol{a}}$ for frequency-wavenumber combinations near isolated, repeated or clustered apex eigenfrequencies.

Isolated eigenvalue. This case is illustrated in Fig. 10, for the brickwall example with $10 \%$ ligament length and frequency-wavenumber combinations $(23)\left(\right.$ with $\left.\sigma \hat{\omega}^{2}=0\right)$ near the isolated eigenvalue $\tilde{\lambda}_{n}^{a}(n=7)$ at apex C. In the display, the second-order FW-FF approximation of $\langle\tilde{u}\rangle_{\boldsymbol{a}}$ given by Theorem 1 and its zeroth- and first-order companions are compared over a range of values of $\epsilon$ to the reference value of $\langle\tilde{u}\rangle_{\boldsymbol{a}}$ found from applying definition (20) to the numerically computed solution of the forced vibration problem (24)-(25). A good agreement is observed, and the resulting relative differences (in $L^{2}(Y)$ norm) again scale as expected with $\epsilon$.

Repeated eigenvalue. We consider in Fig. 11 a double eigenfrequency occurring for $n=10,11$ at apex C for the disk example with $\rho_{1}=5 \rho_{2}$ and $\kappa=10$. The leading-order approximation of the mean motion components $\langle\tilde{u}\rangle_{\boldsymbol{a}}^{q}(q=1,2)$, defined by (65) and computed for the diagonalizing projection basis (see Remark 20), is quantitatively compared (again by means of relative differences in $L^{2}(Y)$ norm) with numerically computed reference values in Fig. 11. As can be seen from the display, the $L^{2}(Y)$-discrepancy between the asymptotic model and its reference counterpart vanishes roughly as $O(\epsilon)$, as may be expected for a leading-order local approximation. 


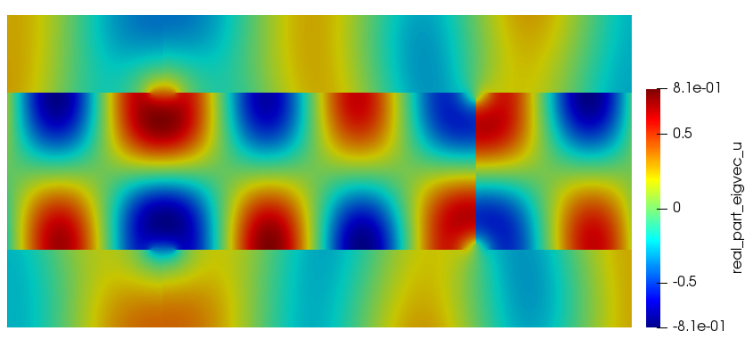

(a) $\operatorname{Re}\left(\tilde{\varphi}_{n, \epsilon}^{\boldsymbol{a}, \mathrm{ref}}\right)$ for $\hat{\boldsymbol{k}}=\hat{\boldsymbol{k}}_{1}$

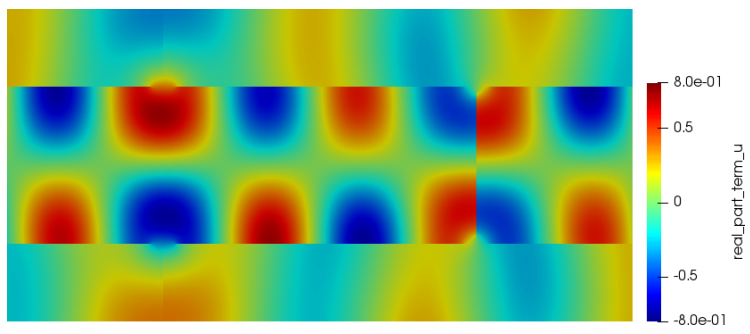

(c) $\operatorname{Re}\left(\tilde{\varphi}_{n, \epsilon}^{\boldsymbol{a}, \text { asy }}\right)$ for $\hat{\boldsymbol{k}}=\hat{\boldsymbol{k}}_{1}$

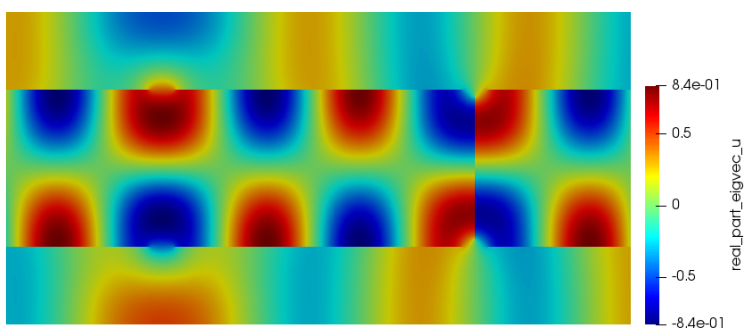

(e) $\operatorname{Re}\left(\tilde{\varphi}_{n}^{a}\right)$

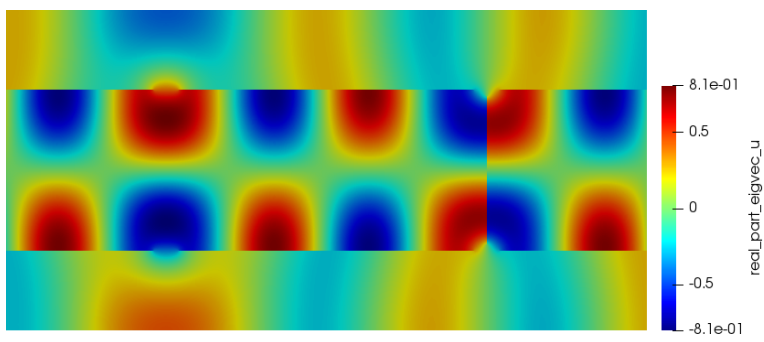

(g) $\operatorname{Re}\left(\tilde{\varphi}_{n, \epsilon}^{\boldsymbol{a}, \mathrm{rref}}\right)$ for $\hat{\boldsymbol{k}}=\hat{\boldsymbol{k}}_{2}$

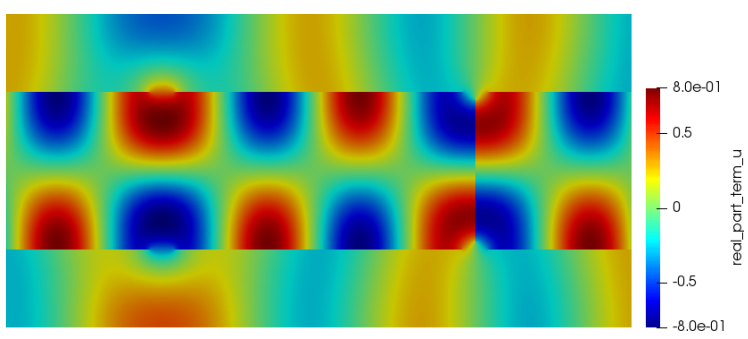

(i) $\operatorname{Re}\left(\tilde{\varphi}_{n, \epsilon}^{\boldsymbol{a}, \text { asy }}\right)$ for $\hat{\boldsymbol{k}}=\hat{\boldsymbol{k}}_{2}$

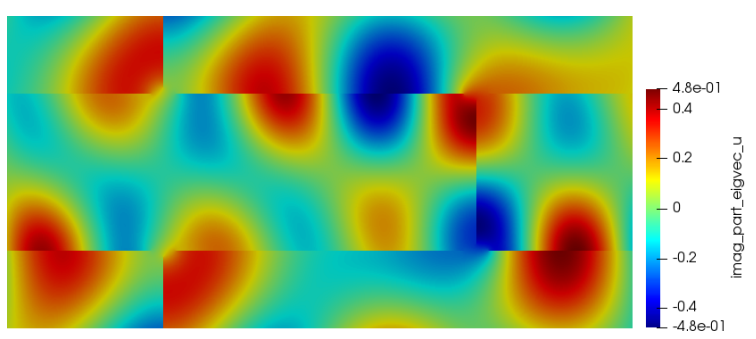

(b) $\operatorname{Im}\left(\tilde{\varphi}_{n, \epsilon}^{\boldsymbol{a}, \mathrm{ref}}\right)$ for $\hat{\boldsymbol{k}}=\hat{\boldsymbol{k}}_{1}$

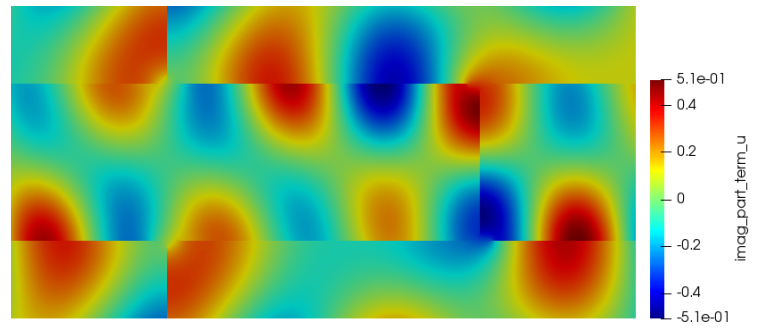

(d) $\operatorname{Im}\left(\tilde{\varphi}_{n, \epsilon}^{\boldsymbol{a}, \text { asy }}\right)$ for $\hat{\boldsymbol{k}}=\hat{\boldsymbol{k}}_{1}$

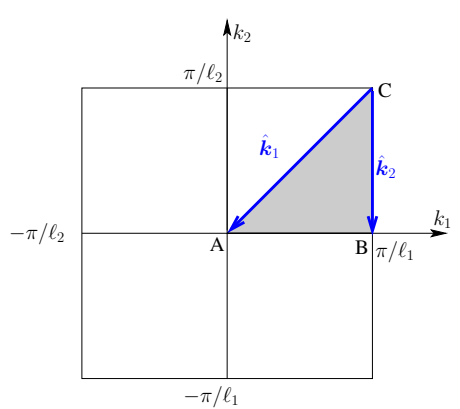

(f) Wavenumber perturbation directions $\hat{\boldsymbol{k}}_{1}, \hat{\boldsymbol{k}}_{2}$ about apex C.

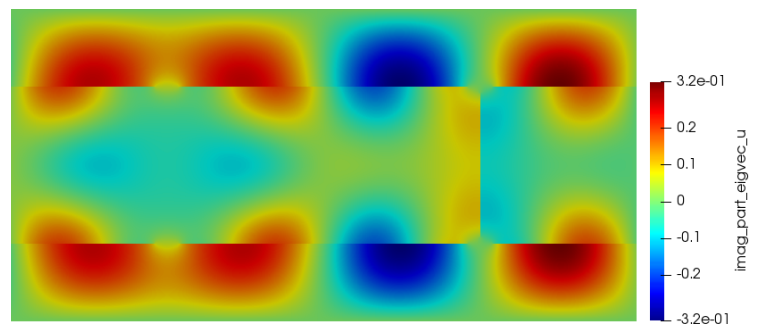

(h) $\operatorname{Im}\left(\tilde{\varphi}_{n, \epsilon}^{\boldsymbol{a}, \mathrm{ref}}\right)$ for $\hat{\boldsymbol{k}}=\hat{\boldsymbol{k}}_{2}$

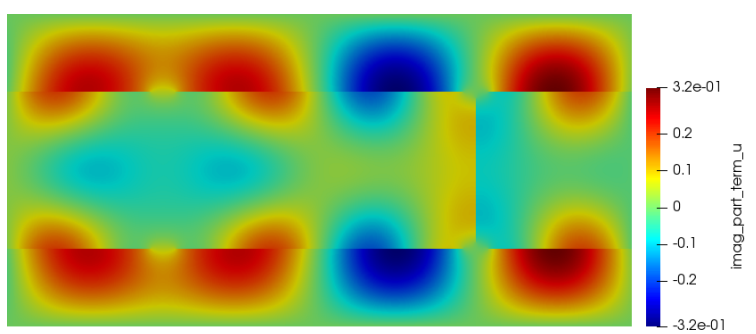

(j) $\operatorname{Im}\left(\tilde{\varphi}_{n, \epsilon}^{\boldsymbol{a}, \text { asy }}\right)$ for $\hat{\boldsymbol{k}}=\hat{\boldsymbol{k}}_{2}$

Figure 7: Brick wall example, $10 \%$ ligament length. Computed reference $\tilde{\varphi}_{n, \epsilon}^{\boldsymbol{a}, \text {,ref }}$ and asymptotic approximation $\tilde{\varphi}_{n, \epsilon}^{\boldsymbol{a} \text {, asy }}$ of eigenfunction $\tilde{\varphi}_{n, \epsilon}^{\boldsymbol{a}}$ with $n=25$ and $\epsilon=0.25$ in scaling (23). Wavenumber perturbation direction $\hat{\boldsymbol{k}}=\hat{\boldsymbol{k}}_{1}$ (first two rows) or $\hat{\boldsymbol{k}}=\hat{\boldsymbol{k}}_{2}$ (last two rows). The corresponding eigenfunction $\tilde{\varphi}_{n}^{a}$ at apex $\mathrm{C}$ (with $\operatorname{Im}\left(\tilde{\varphi}_{n}^{a}\right)=0$ by convention) is shown for comparison in the middle row. 


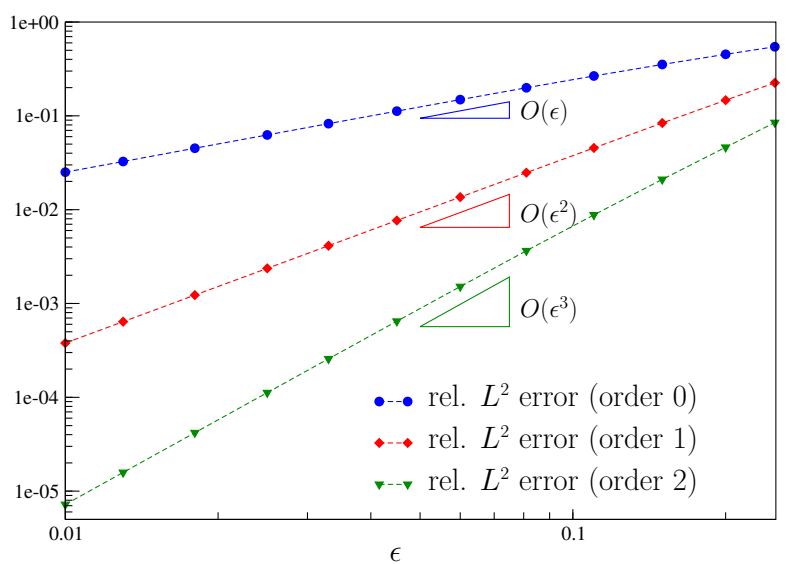

(a) Apex C, $\hat{\boldsymbol{k}}=\hat{\boldsymbol{k}}_{1}$

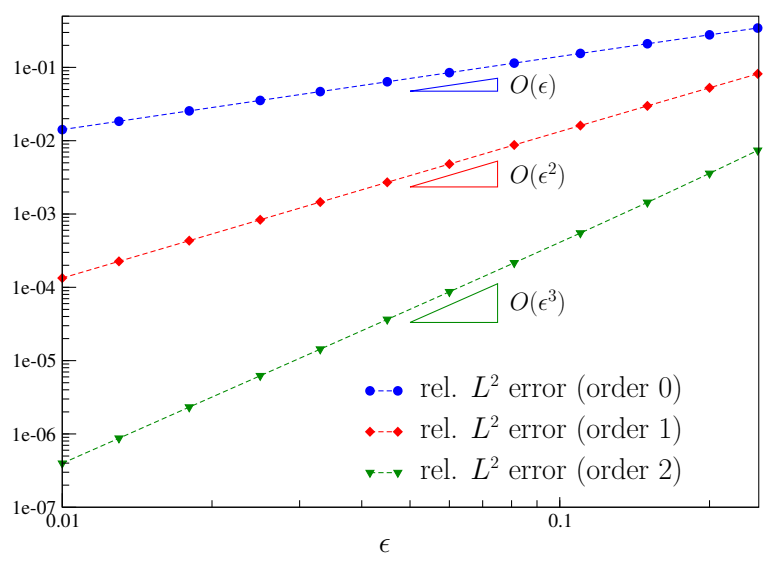

(b) Apex C, $\hat{\boldsymbol{k}}=\hat{\boldsymbol{k}}_{2}$

Figure 8: Brick wall example, $10 \%$ ligament length: relative approximation error on eigenfunction $\tilde{\varphi}_{n, \epsilon}^{a}$ near apex C $(n=25)$, with $\hat{\boldsymbol{k}}_{1}, \hat{\boldsymbol{k}}_{2}$ specified as in Fig. $7 \mathrm{f}$.

Clustered eigenvalues. We finally examine the accuracy of the leading-order approximation of forced motions for wavenumber-frequency combinations that are close to a cluster (99)-(100) of $Q$ nearby frequencies according to

$$
\boldsymbol{k}=\boldsymbol{k}^{\boldsymbol{a}}+\epsilon \hat{\boldsymbol{k}}=\boldsymbol{k}^{\boldsymbol{a}}+\epsilon \zeta \hat{\boldsymbol{k}} /\|\hat{\boldsymbol{k}}\|, \quad \omega^{2}=\tilde{\lambda}_{n}^{a}+\epsilon \sigma \breve{\omega}^{2},
$$

where $\breve{\omega}$ is chosen such that $(\boldsymbol{k}, \omega)$ does not, to leading order, lie on a dispersion branch (see Remark 17) and $\zeta:=\|\hat{\boldsymbol{k}}\|$. The featured components $\langle\tilde{u}\rangle_{p}^{\boldsymbol{a}}$ of the mean motion $(1 \leqslant p \leqslant Q)$ are then evaluated as the weighted averages

$$
\langle\tilde{u}\rangle_{\boldsymbol{a}}^{p} \approx \epsilon_{0}^{-1} \sum_{q=1}^{Q} u_{1 q}(\zeta)\left(\tilde{\varphi}_{j(q)}^{\boldsymbol{a}}, \tilde{\varphi}_{j(p)}^{\boldsymbol{a}}\right)
$$

where, for a fixed perturbation direction, $u_{1 q}=u_{1 q}(\zeta)(q \in \overline{1, Q})$ solve (77). For each example configuration considered in this section, the first 30 computed eigenfrequencies yield several such clusters. For all the numerical results to follow, the "center" eigenvalue $\tilde{\lambda}_{n}^{a}$ is taken as the arithmetic mean of all cluster eigenvalues, namely $\tilde{\lambda}_{n}^{a}=\frac{1}{Q} \sum_{q=1}^{Q} \tilde{\lambda}_{j(q)}^{a}$.

Figs. 12 and 13 plot, for the disk example with either $\rho_{2}=2 \rho_{1}, \kappa=1$ or $\rho_{2}=5 \rho_{1}, \kappa=10$ and several cases of nearby clusters with $Q \in\{2,3,4\}$, the relative error between the approximate mean motions (119) and their reference counterparts (65). In keeping with the discussion in Remark 25, the approximate values $u_{1 p}(\zeta)$ exhibit no discernible dependence on powers of $\zeta$ and merely corroborate the finding that $u_{1 p}(\zeta)$ approximate the respective mean motions near clusters of nearby frequencies within acceptable accuracy for a range of frequency-wavenumber combinations that is commensurate with the eigenfrequency spread of the cluster. To provide a benchmark for future studies, we also provide in Table 2 numerical values of the computed reference and approximated mean motions $\left\{u_{11}, u_{14}\right\}$ corresponding to the relative errors shown in Fig. 13c.

We finally consider the case of an "almost-triple" eigenvalue $(n=2,3,4)$, occurring for the disk configuration with $\rho_{2}=2 \rho_{1}$ and $\kappa=1$ at apex C. For this cluster, we have $\tilde{\lambda}_{2}^{a}, \tilde{\lambda}_{3}^{a} \approx 15.6762$ and $\tilde{\lambda}_{4}^{a} \approx 15.7354$, giving the relative eigenvalue spread $\epsilon \approx 0.001884$. Depending on the range of wavenumber-frequency perturbations, such situation may be treated either as: (i) cluster of nearby eigenvalues, or (ii) repeated (triple) eigenvalue. This is illustrated in Fig. 14, which plots relative errors incurred by the leading-order approximations of effective motions arising from each viewpoint. The asymptotic approximation given by the repeated-eigenfrequency model is seen to start deteriorating when the diminishing magnitude of wavenumber perturbation, $\|\hat{\boldsymbol{k}}\|=\zeta \epsilon$, reaches approximately $0.04 \epsilon$. Below this threshold, the cluster is better approximated via the nearby eigenvalue model as is evident from Fig. 14.

Contribution of a cluster of nearby dispersion branches to the mean energy density. The mean (total) energy density of a Bloch wave (5), averaged in space over $Y_{\boldsymbol{a}}$ and time over $2 \pi / \omega$, is given [34] by $\bar{E}=\frac{1}{2} \omega^{2}(\rho \tilde{u}, \tilde{u})_{Y_{\boldsymbol{a}}}$. 


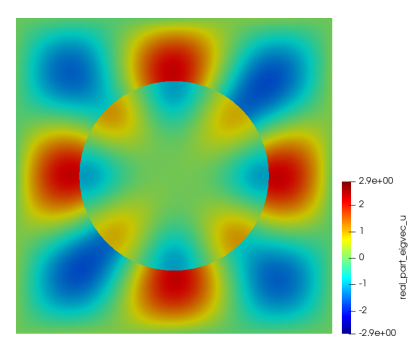

(a) $\operatorname{Re}\left(\tilde{\varphi}_{n, \epsilon}^{\boldsymbol{a}, \mathrm{ref}}\right)$ for $\hat{\boldsymbol{k}}=\hat{\boldsymbol{k}}_{1}$

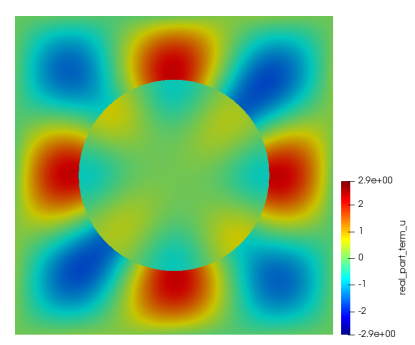

(c) $\operatorname{Re}\left(\tilde{\varphi}_{n, \epsilon}^{a}\right)$ for $\hat{\boldsymbol{k}}=\hat{\boldsymbol{k}}_{1}$

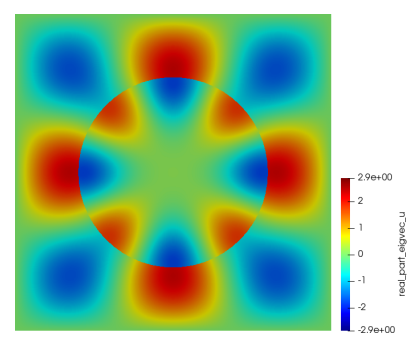

(e) $\operatorname{Re}\left(\tilde{\varphi}_{n}^{a}\right)$

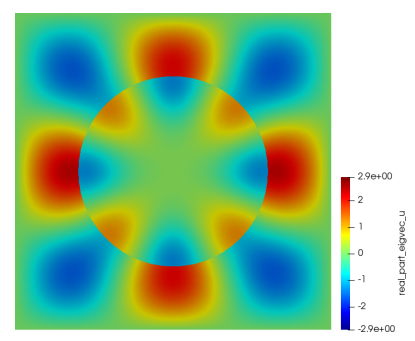

(g) $\operatorname{Re}\left(\tilde{\varphi}_{n, \epsilon}^{\boldsymbol{a}, \mathrm{rre}}\right)$ for $\hat{\boldsymbol{k}}=\hat{\boldsymbol{k}}_{2}$

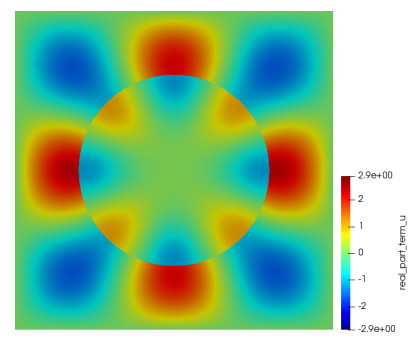

(i) $\operatorname{Re}\left(\tilde{\varphi}_{n, \epsilon}^{\boldsymbol{a}}\right)$ for $\hat{\boldsymbol{k}}=\hat{\boldsymbol{k}}_{2}$

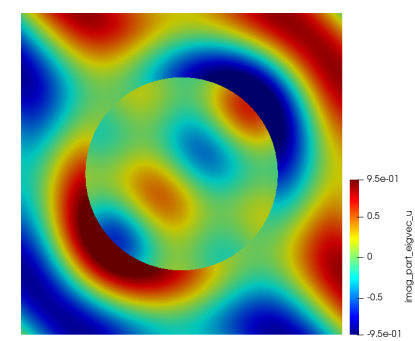

(b) $\operatorname{Im}\left(\tilde{\varphi}_{n, \epsilon}^{\boldsymbol{a}, \mathrm{ref}}\right)$ for $\hat{\boldsymbol{k}}=\hat{\boldsymbol{k}}_{1}$

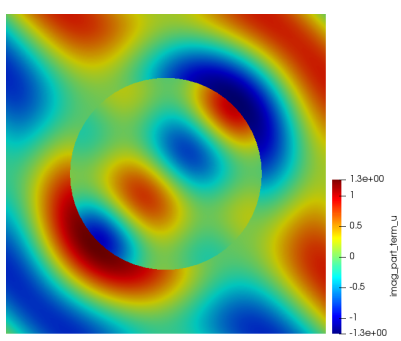

(d) $\operatorname{Im}\left(\tilde{\varphi}_{n, \epsilon}^{a}\right)$ for $\hat{\boldsymbol{k}}=\hat{\boldsymbol{k}}_{1}$

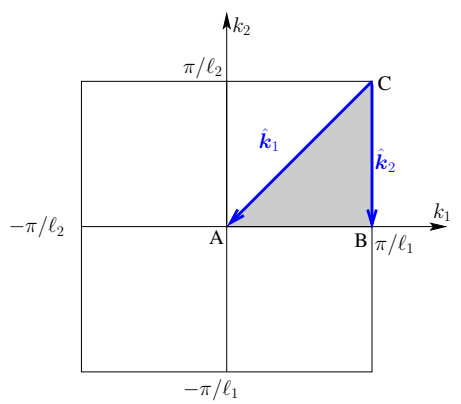

(f) Wavenumber perturbation directions $\hat{\boldsymbol{k}}_{1}, \hat{\boldsymbol{k}}_{2}$ about apex C.

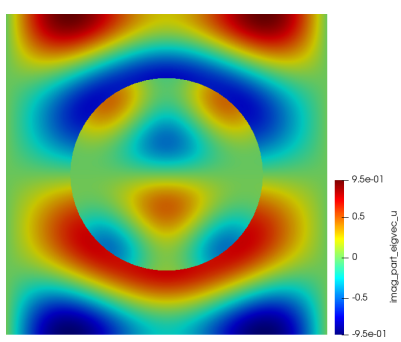

(h) $\operatorname{Im}\left(\tilde{\varphi}_{n, \epsilon}^{\boldsymbol{a}, \mathrm{ref}}\right)$ for $\hat{\boldsymbol{k}}=\hat{\boldsymbol{k}}_{2}$

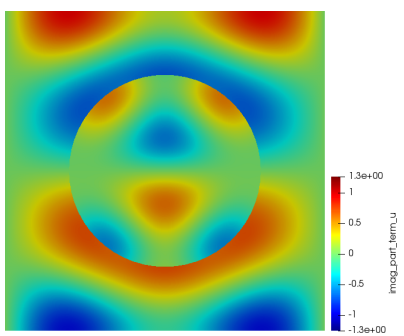

(j) $\operatorname{Im}\left(\tilde{\varphi}_{n, \epsilon}^{a}\right)$ for $\hat{\boldsymbol{k}}=\hat{\boldsymbol{k}}_{2}$

Figure 9: Disk example $\left(\rho_{1}=2, \kappa=1\right)$. Computed reference $\tilde{\varphi}_{n, \epsilon}^{\boldsymbol{a}, \text { ref }}$ and asymptotic approximation $\tilde{\varphi}_{n, \epsilon}^{\boldsymbol{a} \text {, asy }}$ of eigenfunction $\tilde{\varphi}_{n, \epsilon}^{\boldsymbol{a}}$ with $n=24$ and $\epsilon=0.25$ in scaling (23). Wavenumber perturbation direction $\hat{\boldsymbol{k}}=\hat{\boldsymbol{k}}_{1}$ (first two rows) or $\hat{\boldsymbol{k}}=\hat{\boldsymbol{k}}_{2}$ (last two rows). The corresponding eigenfunction $\tilde{\varphi}_{n}^{a}$ at apex $\mathrm{C}$ (with $\operatorname{Im}\left(\tilde{\varphi}_{n}^{a}\right)=0$ by convention) is shown for comparison in the middle row. 


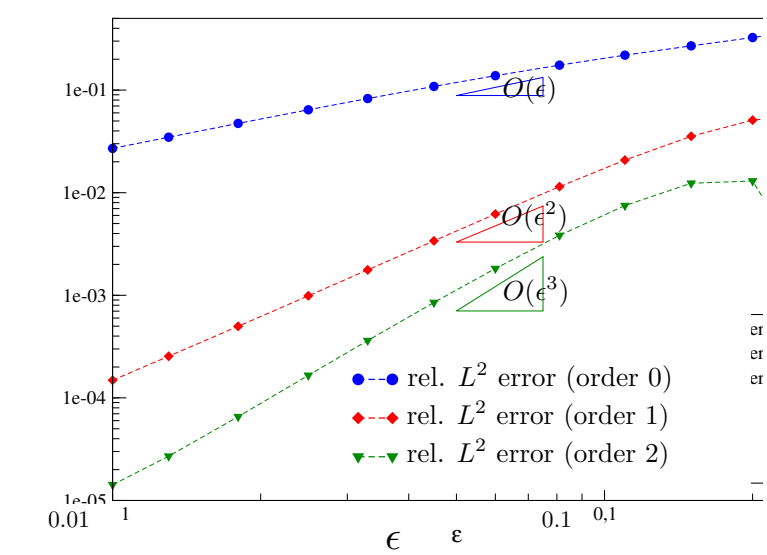

(a) Apex C, $\hat{\boldsymbol{k}}=\hat{\boldsymbol{k}}_{1}$

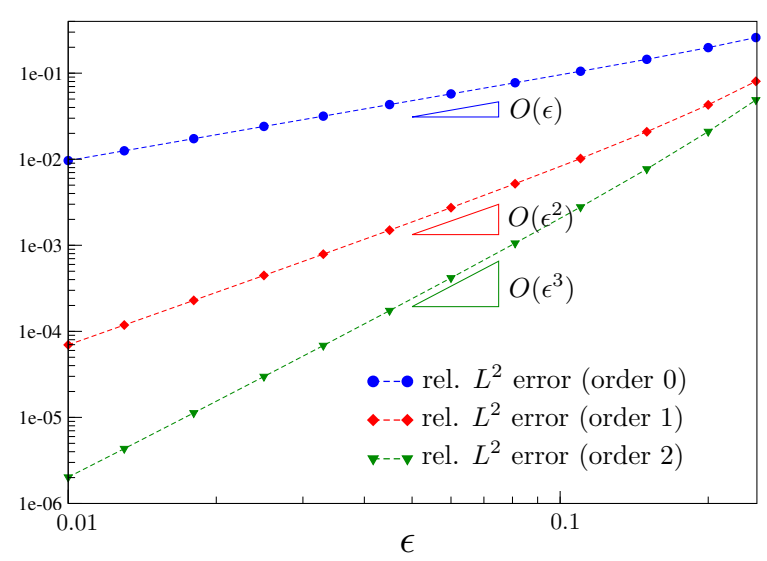

(b) Apex C, $\hat{\boldsymbol{k}}=\hat{\boldsymbol{k}}_{2}$

Figure 10: Brick wall example, $10 \%$ ligament length: relative approximation error on effective motion near apex C $(n=7)$, with $\hat{\boldsymbol{k}}_{1}, \hat{\boldsymbol{k}}_{2}$ specified as in Fig. $7 \mathrm{f}$.

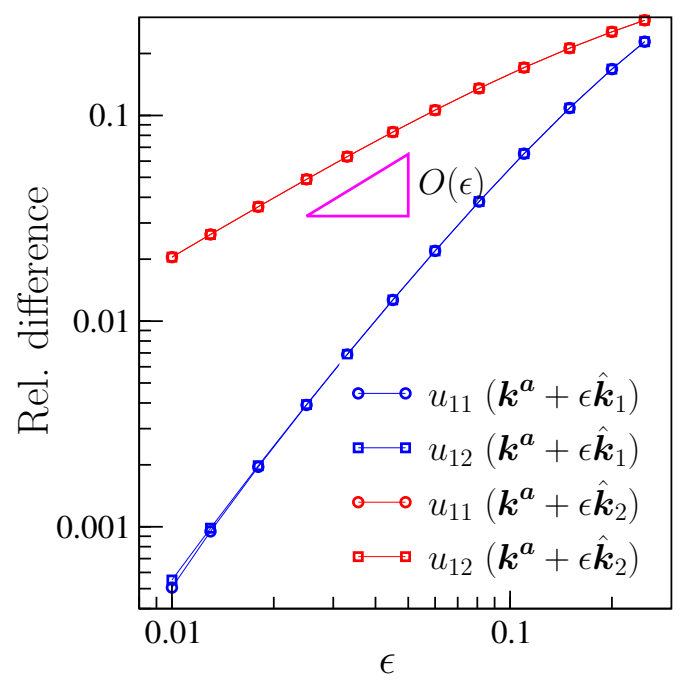

Figure 11: Disk with $\rho_{1}=5 \rho_{2}, \kappa=10$ : relative approximation error on effective motion near apex C (double eigenfrequency, $n=10,11)$, with $\hat{\boldsymbol{k}}_{1}, \hat{\boldsymbol{k}}_{2}$ specified as in Fig. 7 f.

When the driving frequency is close to a cluster of tightly spaced dispersion branches, $\bar{E}$ is predominantly carried by the cluster eigenfunctions; for instance, we find that $\bar{E}=\epsilon^{-4}\left(\frac{1}{2} \omega^{2} \sum_{q=1}^{N} \rho_{q}^{(0)}\left|u_{0 q}\right|^{2}+o(1)\right)$ for the configuration summarized in Remark 22. Table 3 provides computational evidence of such claim by considering the forced vibration problem (24)-(25) for the disk configuration $\left(\rho_{2}=5 \rho_{1}, \kappa=10\right)$ with the source factor $\tilde{f}(\boldsymbol{x})$ in (24) set to $\tilde{f}=\boldsymbol{k}^{\boldsymbol{a}} \cdot \boldsymbol{x}$ and a sample of driving $(\boldsymbol{k}, \omega)$ pairs taken close to the cluster $(6-9)_{\mathrm{N}}$ near apex $\mathrm{C}$ via parametrization $\boldsymbol{k}=\boldsymbol{k}^{a}+\epsilon \hat{\boldsymbol{k}}_{2}$ and $\omega^{2}=\tilde{\lambda}_{6}^{\boldsymbol{a}}(1.025+\epsilon)$.

\section{Conclusions}

In this study, we pursue finite-wavenumber, finite-frequency homogenization of waves in periodic block structures that pertain to e.g. masonry and fractured geomaterials. Following [16] we consider a Bloch-wave form of the scalar wave equation, and we seek an asymptotic expansion about a reference point in the wavenumberfrequency space - using wavenumber separation to define the perturbation parameter. We further deploy the concept of broken Sobolev spaces to cater for the presence of kinematic discontinuities, and we adopt the projection of a Bloch wave onto an eigenfunction for the unit cell of periodicity (at specified eigenfrequency) to 

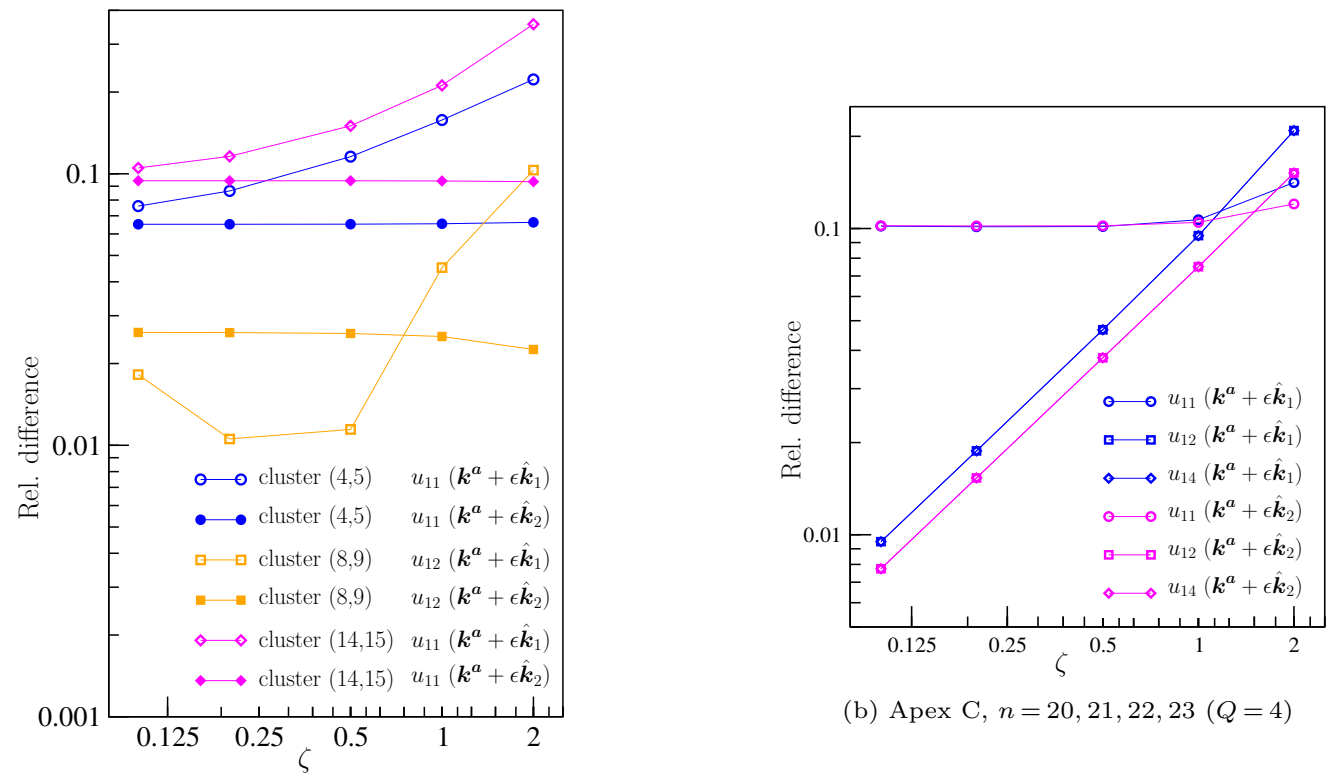

(b) Apex C, $n=20,21,22,23(Q=4)$

(a) Apex B, three nearby clusters with $Q=2$

Figure 12: Disk $\left(\rho_{1}=2, \kappa=1\right)$ : approximation error on effective motion for clusters of nearby eigenvalues, with $\zeta=\|\hat{\boldsymbol{k}}\|$ (see (118)) and $\hat{\boldsymbol{k}}_{1}, \hat{\boldsymbol{k}}_{2}$ specified as in Fig. 2b (a) or Fig. 7f (b). In (a), the approximation errors for the mean motions $u_{11}$ (cluster 8-9) and $u_{12}$ (clusters $4-5$ and 14-15), which are very small, are not shown.

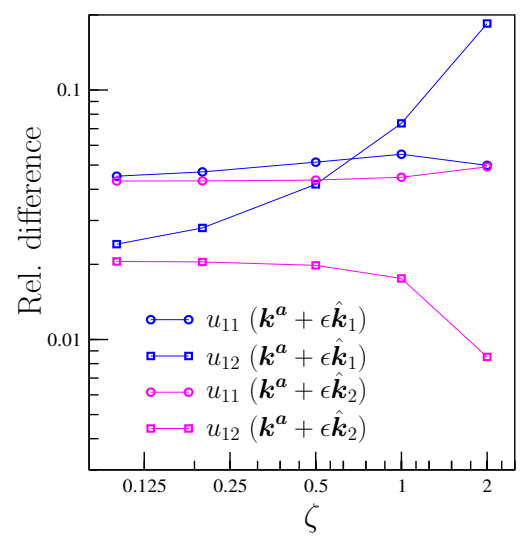

(a) Apex B, $n=14,15,16(Q=3)$

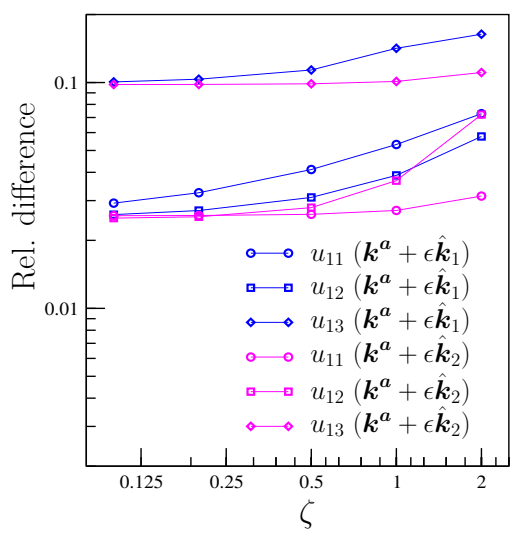

(b) Apex B, $n=20,21,22(Q=3)$

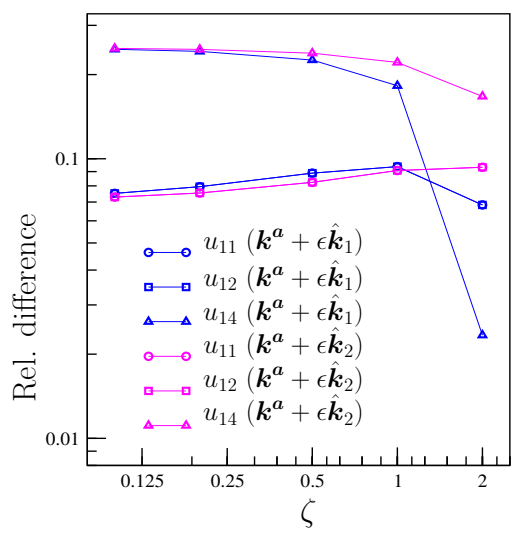

(c) Apex C, $n=12,13,14,15(Q=4)$

Figure 13: Disk $\left(\rho_{1}=5, \kappa=10\right)$ : relative approximation error on effective motion for clusters of nearby eigenvalues, with $\zeta=\|\hat{\boldsymbol{k}}\|$ (see (118)) and $\hat{\boldsymbol{k}}_{1}, \hat{\boldsymbol{k}}_{2}$ specified as in Fig. 2b (a,b) or Fig. 7f (c). In (c), the approximation errors for the mean motions $u_{13}$, which are very small, are not shown.

define the "mean" wave motion. This allows us to distill an effective field equation, for an arbitrary dispersion branch, near apexes of "wavenumber quadrants" featured by the first Brillouin zone. For generality we account for situations featuring both (a) isolated, (b) repeated, and (c) nearby eigenvalues. In the case of repeated eigenvalues, we find that the effective i.e. "mean" wave motion is governed by a system of wave equations and Dirac equations, whose total number is given by the multiplicity of the eigenvalue, and whose structure is given by the spectral properties of a particular matrix whose entries are computed from the participating eigenfunctions, the properties of the unit cell, and the direction of perturbation in the wavenumber space. One of these structures is shown to describe the so-called Dirac points, i.e. conical contacts between dispersion surfaces, that support the generation of topologically protected waves. In situations featuring clusters of tightly spaced eigenvalues, the effective model is found to entail a Dirac-like system of equations that generates "blunted" 


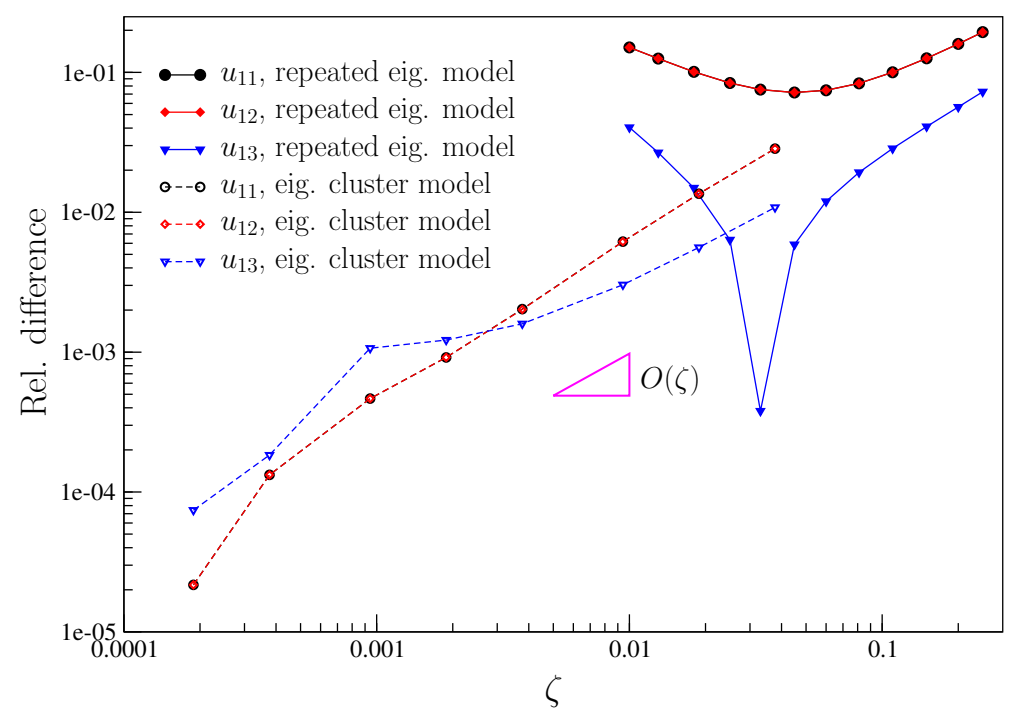

Figure 14: Disk $\left(\rho_{1}=2 \rho_{2}, \kappa=1\right)$ : relative approximation error on effective motion near apex C $(n=2,3,4)$, with $\hat{\boldsymbol{k}}_{1}, \hat{\boldsymbol{k}}_{2}$ as in Fig. 7f and $\zeta=\|\hat{\boldsymbol{k}}\|$ (see (118)). Approximations follow either the nearby-eigenvalue viewpoint (used for $\zeta \leqslant 0.04$ ) or the repeated-eigenvalue viewpoint (used for $\zeta \geqslant 0.01$ ).

\begin{tabular}{|c|c|c|c|c|}
\hline \multirow[t]{2}{*}{$\zeta$} & \multicolumn{2}{|c|}{$u_{11}\left(\boldsymbol{k}^{\boldsymbol{a}}+\epsilon \hat{\boldsymbol{k}}_{1}\right)$} & \multicolumn{2}{|c|}{$u_{14}\left(\boldsymbol{k}^{\boldsymbol{a}}+\epsilon \hat{\boldsymbol{k}}_{1}\right)$} \\
\hline & ref. & asympt. approx. & ref. & asympt. approx. \\
\hline 0.1 & $(-0.0210585,-0.0467813)$ & $(-0.0194747,-0.0432629)$ & $\left(0.0156866,7.8435910^{-9}\right)$ & $\left(0.0118228,9.5498810^{-9}\right)$ \\
\hline 0.2 & $(-0.0211987,-0.0470927)$ & $(-0.0195139,-0.0433500)$ & $\left(0.0150709,7.8257610^{-9}\right)$ & $\left(0.0114225,9.5060010^{-9}\right)$ \\
\hline 0.5 & $(-0.0215269,-0.0478219)$ & $(-0.0196144,-0.0435731)$ & $\left(0.0131880,7.7315310^{-9}\right)$ & $\left(0.0102139,9.3662810^{-9}\right)$ \\
\hline 1. & $(-0.0217636,-0.0483477)$ & $(-0.0197238,-0.0438163)$ & $\left(0.0100105,7.4472310^{-9}\right)$ & $\left(0.0081839,9.1079510^{-9}\right)$ \\
\hline \multirow[t]{3}{*}{2.} & $(-0.0211757,-0.0470404)$ & $(-0.0197276,-0.0438247)$ & $\left(0.0040501,6.5074310^{-9}\right)$ & $\left(0.0041449,8.5083010^{-9}\right)$ \\
\hline & \multicolumn{2}{|c|}{$u_{11}\left(\boldsymbol{k}^{a}+\epsilon \hat{\boldsymbol{k}}_{2}\right)$} & \multicolumn{2}{|c|}{$u_{14}\left(\boldsymbol{k}^{\boldsymbol{a}}+\epsilon \hat{\boldsymbol{k}}_{2}\right)$} \\
\hline & ref. & asympt. approx. & ref. & asympt. approx. \\
\hline 0.1 & $(-0.0211762,-0.0465305)$ & $(-0.0194995,-0.0432010)$ & $\left(0.0159902,7.8905810^{-9}\right)$ & $\left(0.0120221,9.6357610^{-9}\right)$ \\
\hline 0.2 & $(-0.0214322,-0.0466305)$ & $(-0.0195599,-0.0432426)$ & $\left(0.0156816,8.0023610^{-9}\right)$ & $\left(0.0118219,9.7279910^{-9}\right)$ \\
\hline 0.5 & $(-0.0220973,-0.0469676)$ & $(-0.0197022,-0.0434281)$ & $\left(0.0147319,8.7900810^{-9}\right)$ & $\left(0.0112168,1.0299410^{-8}\right)$ \\
\hline 1. & $(-0.0228498,-0.0476528)$ & $(-0.0198068,-0.0439454)$ & $\left(0.0130903,1.1596810^{-8}\right)$ & $\left(0.0101954,1.2256910^{-8}\right)$ \\
\hline 2. & $(-0.0229751,-0.0495659)$ & $(-0.0194760,-0.0458646)$ & $\left(0.0097554,2.2708910^{-8}\right)$ & $\left(0.0081252,2.0319210^{-8}\right)$ \\
\hline
\end{tabular}

Table 2: Disk example $\left(\rho_{2}=5 \rho_{1}, \kappa=10\right)$ : (complex) numerical values of the reference mean motions $\left\{u_{11}, u_{14}\right\}$ and their asymptotic approximations (corresponding to the relative errors shown in Fig. 13c), with $\zeta=\|\hat{\boldsymbol{k}}\|\left(\right.$ see $(118)$ ) and $\hat{\boldsymbol{k}}_{1}, \hat{\boldsymbol{k}}_{2}$ specified as in Fig. 7f.

\begin{tabular}{|l|c||c|}
\hline$\epsilon$ & $\bar{E}$ & Relative cluster contribution \\
\hline 0.01 & 66.2 & $.233+.214+.537+.013=.997$ \\
0.02 & 25.7 & $.373+.336+.238+.046=.992$ \\
0.05 & 37.2 & $.086+.082+.010+.817=.995$ \\
0.1 & 3.64 & $.270+.278+.037+.365=.950$ \\
\hline
\end{tabular}

Table 3: Disk example $\left(\rho_{2}=5 \rho_{1}, \kappa=10\right)$ : relative contribution of the nearby dispersion branches to the overall mean energy density, $\bar{E}=\frac{1}{2} \omega^{2}(\rho \tilde{u}, \tilde{u})_{Y_{\boldsymbol{a}}}$. Driving wavenumber-frequency combinations, indicated by dots in Fig. $6 \mathrm{a}$, are close to the cluster $(6-9)_{\mathrm{N}}$ near apex C.

conical dispersion surfaces. We illustrate the analytical developments by numerical simulations for two example configurations in $\mathbb{R}^{2}$ that (i) showcase the metamaterial design using soft interfaces, and (ii) demonstrate the occurence of band gaps and Dirac-like dispersion structures in masonry-like discontinua. From the practical point of view, this work is expected to enrich the manufacturing (e.g. 3D printing) of phononic crystals 
and metamaterials, for it allows a spectrum of material parameters - in this case specific stiffness coefficients - to be manufactured (and tightly controlled) using only a single material phase, e.g. polymer.

\section{Acknowledgments}

The efforts of BBG during the course of this investigation were partially supported through the endowed Shimizu Professorship. The support by POEMS, ENSTA Paris which allowed the first author to visit MB during the summer of 2019 is kindly acknowledged.

\section{Appendix}

Proof of Lemma 1: On adding (18) to their respective complex conjugates and recalling that $G, \rho, \kappa$ and $\tilde{\lambda}_{n}^{a}$ are real valued, we find that

$$
\begin{aligned}
& -\nabla \cdot\left(G \nabla\left(\tilde{\varphi}_{n}^{a}+\overline{\varphi_{n}^{a}}\right)\right)=\tilde{\lambda}_{n}^{a} \rho\left(\tilde{\varphi}_{n}^{a}+\tilde{\varphi}_{n}^{a}\right) \quad \text { in } Y_{\boldsymbol{a}},
\end{aligned}
$$

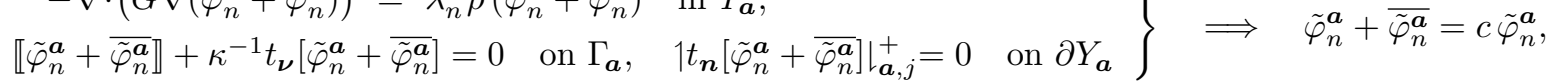

where $c$ is a constant. Accordingly, $\arg \left(c \tilde{\varphi}_{n}^{a}(\boldsymbol{x})\right)=0$ and letting $c \in \mathbb{R}$, we have $\operatorname{Im}\left(\tilde{\varphi}_{n}^{\boldsymbol{a}}\right)=0$.

Proof of Lemma 2: By premise that $\tilde{\lambda}_{n}$ is a simple eigenvalue, $\tilde{\phi}_{n}^{a} \in H_{p}^{1}(Y)$ is the only eigenfunction solving (14). By construction, $\tilde{\varphi}_{n}^{a} \in H_{p}^{1}\left(Y_{\boldsymbol{a}}\right)$ is the only solution of (18) that include the $Y$-antiperiodicity constraint given by the third equation. As a result any solution to $(33)-(34)$ in $\left(H_{p 0}^{1 \boldsymbol{a}}\left(Y_{\boldsymbol{a}}\right)\right)^{d}$, subject to the implicit constraint

due to $(27)$, is unique.

$$
\left.a_{j} \chi^{(1)}\right|_{x_{j}=0}=-\left.a_{j} \chi^{(1)}\right|_{x_{j}=\ell_{j}}
$$

To demonstrate the existence of a solution to (33)-(34), it suffices to show that the source term in (33) is, subject to germane boundary and jump conditions, orthogonal to $\tilde{\varphi}_{n}^{a}$. To this end, we make use of the fact that $\tilde{\varphi}_{n}^{a}$ is real-valued and integrate the product of the left-hand side of (33) and $\varphi_{\boldsymbol{a}}$ by parts as

$$
\begin{aligned}
& P=\left(\tilde{\lambda}_{n}^{a} \rho \boldsymbol{\chi}^{(1)}+\nabla \cdot\left(G\left(\nabla \chi^{(1)}+\boldsymbol{I} \tilde{\varphi}_{n}^{a}\right)\right)+G \nabla \tilde{\varphi}_{n}^{a}, \tilde{\varphi}_{n}^{a}\right)_{Y_{a}} \\
& =\left(\nabla \cdot\left(G\left(\nabla \boldsymbol{\chi}^{(1)}+\boldsymbol{I} \tilde{\varphi}_{n}^{\boldsymbol{a}}\right) \tilde{\varphi}_{n}^{\boldsymbol{a}}\right), 1\right)_{Y_{\boldsymbol{a}}}-\left(G \nabla \tilde{\varphi}_{n}^{\boldsymbol{a}} \cdot \nabla \boldsymbol{\chi}^{(1)}, 1\right)_{Y_{\boldsymbol{a}}} \\
& =\left(\nabla \cdot\left(G\left(\nabla \chi^{(1)}+\boldsymbol{I} \tilde{\varphi}_{n}^{\boldsymbol{a}}\right) \tilde{\varphi}_{n}^{\boldsymbol{a}}\right), 1\right)_{Y_{\boldsymbol{a}}}-\left(\nabla \cdot\left(G \nabla \tilde{\varphi}_{n}^{\boldsymbol{a}} \otimes \boldsymbol{\chi}^{(1)}\right), 1\right)_{Y_{\boldsymbol{a}}}+\left(\left(-\tilde{\lambda}_{n}^{a} \rho \tilde{\varphi}_{n}^{a}\right) \chi^{(1)}, 1\right)_{Y_{a}} 0 \\
& =\left(\boldsymbol{t}_{\boldsymbol{n}}^{I}\left[\chi^{(1)}, \tilde{\varphi}_{n}^{a}\right], \tilde{\varphi}_{n}^{a}\right)_{\sum_{\mathfrak{m}=1}^{\mathfrak{M}_{a}} \partial Y_{\mathfrak{m}}}-\left(t_{\boldsymbol{n}}\left[\tilde{\varphi}_{n}^{a}\right], \chi^{(1)}\right)_{\sum_{\mathfrak{m}=1}^{\mathfrak{M}_{a}} \partial Y_{\mathfrak{m}}}
\end{aligned}
$$

where $\boldsymbol{n}_{\mathfrak{m}}$ is the unit outward normal to $\partial Y_{\mathfrak{m}}$, and $t_{\boldsymbol{n}}[g]$ is defined by (3) for either scalar or vector $g$. On deploying the jump conditions in (19) and (34) together with the boundary conditions (34), we find that

$$
P=-\left(\boldsymbol{t}_{\nu}^{I}\left[\boldsymbol{\chi}^{(1)}, \tilde{\varphi}_{n}^{a}\right], \llbracket \tilde{\varphi}_{n}^{a} \rrbracket\right)_{\Gamma_{a}}+\left(t_{\nu}\left[\tilde{\varphi}_{n}^{\boldsymbol{a}}\right], \llbracket \chi^{(1)} \rrbracket\right)_{\Gamma_{a}}=\left(\kappa \llbracket \chi^{(1)} \rrbracket, \llbracket \tilde{\varphi}_{n}^{a} \rrbracket\right)_{\Gamma_{a}}-\left(\kappa \llbracket \tilde{\varphi}_{n}^{a} \rrbracket, \llbracket \chi^{(1)} \rrbracket\right)_{\Gamma_{a}}=0,
$$

which establishes the existence of the solution.

To show that $\chi^{(1)}$ is real-valued, we first note that all the coefficients, together with all (domain and jump) source terms, in (33)-(34) are real-valued. Accordingly, one finds by subtracting (33)-(34) from its complex conjugate that

$$
\chi^{(1)}-\overline{\chi^{(1)}}=c \tilde{\varphi}_{n}^{a}
$$

where overline denotes complex conjugation and $\boldsymbol{c}$ is a constant. Recalling that $\tilde{\varphi}_{n}^{\boldsymbol{a}} \in H_{p}^{1}\left(Y_{\boldsymbol{a}}\right)$, however, we have that $\boldsymbol{c}=\left\langle\boldsymbol{c} \tilde{\varphi}_{n}^{\boldsymbol{a}}\right\rangle_{\boldsymbol{a}}^{\boldsymbol{\varphi}}=\left\langle\chi^{(1)}-\overline{\chi^{(1)}}\right\rangle_{\boldsymbol{a}}^{\varphi}=\left\langle\chi^{(1)}\right\rangle_{\boldsymbol{a}}^{\varphi}-\left\langle\overline{\chi^{(1)}}\right\rangle_{\boldsymbol{a}}^{\varphi}=\mathbf{0}$, which establishes the claim.

Proof of Lemma 3: This proof essentially follows that of Lemma 5 in [16], and is included here for completeness. To establish the claim we integrate the inner product $\left((43), \boldsymbol{\chi}^{(1)}\right)_{Y_{a}}$ by parts as

$$
\tilde{\lambda}_{n}^{a}\left(\rho \eta^{(0)}, \boldsymbol{\chi}^{(1)}\right)_{Y_{\boldsymbol{a}}}-\left(G \nabla \eta^{(0)} \cdot \nabla \boldsymbol{\chi}^{(1)}, 1\right)_{Y_{\boldsymbol{a}}}-\left(t_{\boldsymbol{\nu}}\left[\eta^{(0)}\right], \llbracket \chi^{(1)} \rrbracket\right)_{\Gamma_{\boldsymbol{a}}}=\left(\frac{\rho}{\rho^{(0)}}\left\langle\tilde{f} e^{i \boldsymbol{k}^{\boldsymbol{a}} \cdot \boldsymbol{x}}\right\rangle_{\boldsymbol{a}}^{\varphi} \tilde{\varphi}_{n}^{\boldsymbol{a}}-\tilde{f} e^{i \boldsymbol{k}^{\boldsymbol{a}} \cdot \boldsymbol{x}}, \chi^{(1)}\right)_{Y_{\boldsymbol{a}}} \cdot
$$


Using (33)-(34) and integrating by parts, the second term on the left-hand side becomes

$$
\begin{aligned}
-\left(G \nabla \eta^{(0)} \cdot \nabla \boldsymbol{\chi}^{(1)}, 1\right)_{Y_{a}}=-\left(\nabla \eta^{(0)} \cdot G\left(\nabla \boldsymbol{\chi}^{(1)}+\boldsymbol{I} \tilde{\varphi}_{n}^{\boldsymbol{a}}\right), 1\right)_{Y_{\boldsymbol{a}}}+\left(\nabla \eta^{(0)} G \tilde{\varphi}_{n}^{\boldsymbol{a}}, 1\right)_{Y_{\boldsymbol{a}}} \\
=\left(\eta^{(0)} \nabla \cdot\left(G\left(\nabla \boldsymbol{\chi}^{(1)}+\boldsymbol{I} \tilde{\varphi}_{n}^{a}\right)\right), 1\right)_{Y_{\boldsymbol{a}}}+\left(\nabla \eta^{(0)} G \tilde{\varphi}_{n}^{a}, 1\right)_{Y_{\boldsymbol{a}}}+\left(\boldsymbol{t}_{\boldsymbol{\nu}}^{\boldsymbol{I}}\left[\boldsymbol{\chi}^{(1)}, \tilde{\varphi}_{n}^{\boldsymbol{a}}\right], \llbracket \eta^{(0)} \rrbracket\right)_{\Gamma_{a}} \\
\quad=-\tilde{\lambda}_{n}^{\boldsymbol{a}}\left(\eta^{(0)} \rho \boldsymbol{\chi}^{(1)}, 1\right)_{Y_{\boldsymbol{a}}}-\left(\eta^{(0)} G \nabla \tilde{\varphi}_{n}^{\boldsymbol{a}}, 1\right)_{Y_{\boldsymbol{a}}}+\left(\nabla \eta^{(0)} G \tilde{\varphi}_{n}^{\boldsymbol{a}}, 1\right)_{Y_{\boldsymbol{a}}}+\left(\boldsymbol{t}_{\boldsymbol{\nu}}^{I}\left[\boldsymbol{\chi}^{(1)}, \tilde{\varphi}_{n}^{\boldsymbol{a}}\right], \llbracket \eta^{(0)} \rrbracket\right)_{\Gamma_{\boldsymbol{a}}} .
\end{aligned}
$$

By this result, the interfacial conditions in (19) and (34), and the fact that $\tilde{\varphi}_{n}^{a}$ and $\chi^{(1)}$ are real-valued, (120) recovers (46).

Proof of Lemma 4: By solving (39) for $\sigma \hat{\omega}^{2} u_{0}$ and substituting the result into (50), we obtain

$$
-\left(\boldsymbol{\mu}^{(0)}:(i \hat{\boldsymbol{k}})^{2}+\sigma \rho^{(0)} \hat{\omega}^{2}\right) u_{1}-\left(\boldsymbol{\mu}^{(1)}-\frac{1}{\rho^{(0)}}\left\{\boldsymbol{\rho}^{(1)} \otimes \boldsymbol{\mu}^{(0)}\right\}\right):(i \hat{\boldsymbol{k}})^{3} u_{0}=-\left(\boldsymbol{\chi}^{(1)} \tilde{f} e^{i \boldsymbol{k}^{\boldsymbol{a}} \cdot \boldsymbol{x}}, 1\right)_{\overline{Y_{\boldsymbol{a}}}} \cdot i \hat{\boldsymbol{k}} .
$$

On recalling the field equations (33) and (41) governing $\chi^{(1)}$ and $\chi^{(2)}$, however, one can conveniently integrate by parts their (symmetrized) weighted difference $\left(\left\{(41) \otimes \chi^{(1)}-(33) \otimes \chi^{(2)}\right\}, 1\right)_{\bar{Y}_{a}}$. Noting specifically that

$$
\begin{aligned}
\left(\left\{\nabla \cdot\left(G\left(\nabla \boldsymbol{\chi}^{(2)}+\left\{\boldsymbol{I} \otimes \boldsymbol{\chi}^{(1)}\right\}^{\prime}\right)\right) \otimes \boldsymbol{\chi}^{(1)}\right\}, 1\right)_{Y_{a}}= & -\left(\left\{G \nabla \boldsymbol{\chi}^{(1)} \cdot \nabla \boldsymbol{\chi}^{(2)}\right\}, 1\right)_{Y_{a}}-\left(\left\{G \nabla \boldsymbol{\chi}^{(1)} \otimes \boldsymbol{\chi}^{(1)}\right\}, 1\right)_{Y_{a}} \\
& -\left(\left\{\boldsymbol{t}_{\boldsymbol{\nu}}\left[\boldsymbol{\chi}^{(2)}, \boldsymbol{\chi}^{(1)}\right]\right\}^{\prime} \otimes \llbracket \boldsymbol{\chi}^{(1)} \rrbracket, 1\right)_{\Gamma_{a}}, \\
\left(\left\{\nabla \cdot\left(G\left(\nabla \boldsymbol{\chi}^{(1)}+\boldsymbol{I} \tilde{\varphi}_{n}^{a}\right)\right) \otimes \boldsymbol{\chi}^{(2)}\right\}, 1\right)_{Y_{a}}= & -\left(\left\{G \nabla \boldsymbol{\chi}^{(1)} \cdot \nabla \boldsymbol{\chi}^{(2)}\right\}, 1\right)_{Y_{a}}-\left(\left\{G \nabla \boldsymbol{\chi}^{(2)} \tilde{\varphi}_{n}^{a}\right\}, 1\right)_{Y_{a}} \\
& -\left(\left\{\boldsymbol{t}_{\boldsymbol{\nu}}\left[\boldsymbol{\chi}^{(1)}, \tilde{\varphi}_{n}^{\boldsymbol{a}}\right] \otimes \llbracket \boldsymbol{\chi}^{(2)} \rrbracket\right\}^{\prime}, 1\right)_{\Gamma_{a}},
\end{aligned}
$$

and making use of the interfacial conditions in (34) and (42), we obtain $\rho^{(0)} \boldsymbol{\mu}^{(1)}=\left\{\boldsymbol{\rho}^{(1)} \otimes \boldsymbol{\mu}^{(0)}\right\}$ due to the fact that $\tilde{\varphi}_{n}^{a}$ is real-valued. A substitution of the last result into (121) immediately recovers (52).

Proof of Lemma 5: To establish part (a), we integrate (i) $(33) \otimes \boldsymbol{\chi}^{(3)}$ and (ii) (53) $\otimes \boldsymbol{\chi}^{(1)}$ by parts over $Y_{a}$, evaluate the combination (i)-(ii) of the obtained equalities, and apply the index averaging (28) to the result. Upon recalling definition (56), the latter identity is readily found to provide the claimed alternative expression of $\boldsymbol{\mu}^{(2)}$.

Part (b) is established by a similar method. We integrate (33) $\otimes \boldsymbol{\eta}^{(1)}$, (ii) (41) $\eta^{(0)}$, (iii) $(43) \otimes \boldsymbol{\chi}^{(2)}$ and (iv) $(54) \otimes \chi^{(1)}$ by parts over $Y_{a}$, evaluate the combination (i)-(ii)+(iii)-(iv) of the resulting equalities and apply index averaging. Using thus obtained identity in $F(\hat{\boldsymbol{k}}, \hat{\omega}, \epsilon)$ according to Theorem 1 to eliminate the terms featuring $\boldsymbol{\eta}^{(1)}$ yields the claimed alternative expression.

Proof of Lemma 6: We integrate $(82) \otimes \boldsymbol{\chi}_{p}^{(1)}$ by parts over $Y_{a}$ to obtain

$$
\begin{aligned}
& \quad\left(G \tilde{\varphi}_{n q}^{a}, \nabla \boldsymbol{\chi}_{p}^{(1)}\right)_{\bar{Y}_{a}}-\left(G \nabla \tilde{\varphi}_{n q}^{a}, \boldsymbol{\chi}_{p}^{(1)}\right)_{\bar{Y}_{a}} \\
& \quad=\tilde{\lambda}_{n}\left(\rho \boldsymbol{\chi}_{q}^{(1)} \otimes \boldsymbol{\chi}_{p}^{(1)}, 1\right)_{\bar{Y}_{a}}-\left(G \nabla \boldsymbol{\chi}_{q}^{(1)}, \nabla \boldsymbol{\chi}_{p}^{(1)},\right)_{\bar{Y}_{a}}-\kappa\left(\llbracket \chi_{q}^{(1)} \rrbracket, \llbracket \boldsymbol{\chi}_{p}^{(1)} \rrbracket\right)_{\bar{Y}_{a}}-\sum_{r} \frac{1}{\rho_{r}^{(0)}} \boldsymbol{\theta}_{r q}^{(0)}\left(\rho \tilde{\varphi}_{n r}^{a}, \boldsymbol{\chi}_{p}^{(1)}\right) .
\end{aligned}
$$

The proof then follows from (84) upon rewriting $\boldsymbol{\mu}_{p q}^{(0)}$ as

$$
\boldsymbol{\mu}_{p q}^{(0)}=\left(G\left\{\tilde{\varphi}_{n p}^{a} \nabla \boldsymbol{\chi}_{q}^{(1)}\right\}, 1\right)_{\bar{Y}_{a}}-\left(G\left\{\nabla \tilde{\varphi}_{n p}^{a} \otimes \boldsymbol{\chi}_{q}^{(1)}\right\}, 1\right)_{\bar{Y}_{a}}+\boldsymbol{I}\left(G \tilde{\varphi}_{n p}^{a} \tilde{\varphi}_{n q}^{a}, 1\right)_{\bar{Y}_{a}},
$$

since the first three terms on the RHS of (122) are symmetric in $(p, q)$, while the latter vanishes (to the leading order) upon contraction with $(i \hat{\boldsymbol{k}})^{2}$ due to the fact that each $A_{p q}=\boldsymbol{\theta}_{r q}^{(0)} \cdot(i \hat{\boldsymbol{k}})$ is near-trivial.

Proof of (117): We adapt the derivations of Sections 3.5, 3.6 and 3.7 for the source-free case: using (30), (32), and (45) with $\eta^{(0)}=0$ in (26), we obtain

$$
\tilde{\varphi}_{n, \epsilon}^{\boldsymbol{a}, \mathrm{ref}}=\left(u_{0}+\epsilon u_{1}+\epsilon^{2} u_{2}\right)\left(\tilde{\varphi}_{n}^{\boldsymbol{a}}+\epsilon \boldsymbol{\chi}^{(1)} \cdot \mathrm{i} \hat{\boldsymbol{k}}+\epsilon^{2} \boldsymbol{\chi}^{(2)}:(\mathrm{i} \hat{\boldsymbol{k}})^{2}\right)+o\left(\epsilon^{2}\right) .
$$

The claim (117) follows from evaluating the constant $u_{0}+\epsilon u_{1}+\epsilon^{2} u_{2}$ (up to a unit-norm complex factor) that meets the normalization condition (11) up to order $O\left(\epsilon^{2}\right)$ ). 


\section{References}

[1] A. Anthoine. Derivation of the in-plane elastic characteristics of masonry through homogenization theory. Int. J. Solids Struct., 32:137-163, 1995.

[2] M.W. Ashraf and M. Faryad. Dirac-like cone dispersion in two-dimensional core-shell dielectric photonic crystals. J. Nanophotonics, 9:093057, 2015.

[3] A. Bacigalupo and L. Gambarotta. Computational two-scale homogenization of periodic masonry: characteristic lengths and dispersive waves. Comp Meth. Appl. Mech. Eng., 213:16-28, 2012.

[4] A. Bensoussan, J.-L. Lions, and G. Papanicolaou. Asymptotic Analysis for Periodic Structures, volume 374. 2011.

[5] M.S. Birman and T.A. Suslina. Homogenization of a multidimensional periodic elliptic operator in a neighborhood of the edge of an internal gap. J. Math. Sci., 136:3682-3690, 2006.

[6] M. Brajanovski, B. Gurevich, and M. Schoenberg. A model for P-wave attenuation and dispersion in a porous medium permeated by aligned fractures. Geophys. J. Int., 163:372-384, 2005.

[7] L. Brillouin. Wave Propagation in Periodic Structures. Dover, 1953.

[8] A. Buffa and C. Ortner. Compact embeddings of broken Sobolev spaces and applications. IMA J. Numer. Anal., 29:827-855, 2009.

[9] R.V. Craster, J. Kaplunov, and A.V. Pichugin. High-frequency homogenization for periodic media. Proc. Roy. Soc. A, 466:2341-2362, 2010.

[10] R.V. Craster, J. Kaplunov, E. Nolde, S. Guenneau (2011). High-frequency homogenization for checkerboard structures: defect modes, ultrarefraction, and all-angle negative refraction. J. Opt. Soc. Am. A 28, 1032-1040, 2011.

[11] E.M Daya, B. Braikat, N. Damil, and M. Potier-Ferry. Continuum modeling for the modulated vibration modes of large repetitive structures. Compt. Rend. Mécanique, 330(5), 2002.

[12] K.B. Dossou, L.C. Botten, R.C. McPhedran, C.G. Poulton, A.A. Asatryan, and C. Martijn de Sterke Shallow defect states in two-dimensional photonic crystals. Phys. Rev. A 77, 133-18, 2008.

[13] A. Drougkas, P. Roca, and C. Molins. Analytical micro-modeling of masonry periodic unit cells - elastic properties. Int. J. Solids Struct., 69:169-188, 2015.

[14] R.J. Galvin and B. Gurevich. Frequency-dependent anisotropy of porous rocks with aligned fractures. Geophys. Prospecting, 63(1), 2015.

[15] W.H. Greub. Linear Algebra. Springer-Verlag, 1981.

[16] B.B. Guzina, S. Meng, and O. Oudghiri-Idrissi. A rational framework for dynamic homogenization at finite wavelengths and frequencies. Proc. Roy. Soc. A, 475:20180547, 2019.

[17] N. Kielbasiewicz, E. Lunéville. XLiFE++: a FEM/BEM multipurpose library. https://uma.ensta-paris.fr/soft/XLiFE++/ (repository) and https://hal.archives-ouvertes.fr/hal01737555 (presentation), 2018.

[18] Y. Lu and A. Srivastava. Level repulsion and band sorting in phononic crystals. J. Mech. Phys. Solids, 111:100-112, 2018.

[19] S. Meng and B.B. Guzina. Aon the dynamic homogenization of periodic media: Willis' approach versus two-scale paradigm. Proc. Roy. Soc. A, 474:20170638, 2017.

[20] S. Meng and B.B. Guzina. On the dynamic homogenization of periodic media: Willis' approach versus two-scale paradigm. Proc. Roy. Soc. A, 474:20170638, 2018.

[21] S.H. Mousavi, A.B. Khanikaev, and Z. Wang. Topologically protected elastic waves in phononic metamaterials. Nature Communications, 6:8682, 2015. 
[22] H.B. Mühlhaus, J. Sulem, and P. Unterreiner. Discrete and continuous models for dry masonry columns. J. Eng. Mech, ASCE, 123:399-403, 1997.

[23] H. Nassar, Q.-C. He, and N. Auffray. Willis' elastodynamic homogenization theory revisited for periodic media. J. Mech. Phys. Solids, 77:158-178, 2015.

[24] A.N. Norris, A.L. Shuvalov, and A.A. Kutsenko. Analytical formulation of three-dimensional dynamic homogenization for periodic elastic systems. Proc. Roy. Soc. A, 468:1629-1651, 2012.

[25] G. Salerno and G. de Felice. Continuum modeling of periodic brickwork. Int. J. Solids Struct., 46:12511267, 2009.

[26] M. Schoenberg and C.M. Sayers. Seismic anisotropy of fractured rock. Geophysics, 60:204-211, 1995.

[27] V. P Smyshlyaev and K.D. Cherednichenko. On rigorous derivation of strain gradient effects in the overall behaviour of periodic heterogeneous media. J. Mech. Phys. Solids, 48:1325-1357, 2000.

[28] I. Stefanou, J. Sulem, and I. Vardoulakis. Three-dimensional Cosserat homogenization of masonry structures: elasticity. Acta Geotechnica, 3:71-83, 2008.

[29] I. Stefanou, J. Sulem, and I. Vardoulakis. Homogenization of interlocking masonry structures using a generalized differential expansion technique. Int. J. Solids Struct., 47:1522-1536, 2010.

[30] J. Sulem and H.-B. Mühlhaus. A continuum model for periodic two-dimensional block structures. Mech. Cohesive-Frict. Mater., 2:31-46, 1997.

[31] Patrizia Trovalusci and Annamaria Pau. Derivation of microstructured continua from lattice systems via principle of virtual works: the case of masonry-like materials as micropolar, second gradient and classical continua. Acta Mechanica, 225:157-177, 2014.

[32] A.A. Vasiliev, S.V. Dmitriev, and A.E. Miroshnichenko. Multi-field continuum theory for medium with microscopic rotations. Int. J. Solids Struct., 42:6245-6260, 2005.

[33] C.H. Wilcox. Theory of Bloch waves. J. d'Analyse Math., 33:146-167, 1978.

[34] J.R. Willis. Negative refraction in a laminate. J. Mech. Phys. Solids, 97:10-18, 2016. 